\title{
Three-Parameter Solutions of the PV Schlesinger-Type Equation near the Point at Infinity and the Monodromy Data
}

\author{
Shun SHIMOMURA
}

Department of Mathematics, Keio University, 3-14-1, Hiyoshi, Kohoku-ku, Yokohama 223-8522, Japan

E-mail: shimomur@math.keio.ac.jp

Received May 01, 2018, in final form October 03, 2018; Published online October 22, 2018 https://doi.org/10.3842/SIGMA.2018.113

\begin{abstract}
For the Schlesinger-type equation related to the fifth Painlevé equation (V) via isomonodromy deformation, we present a three-parameter family of matrix solutions along the imaginary axis near the point at infinity, and also the corresponding monodromy data. Two-parameter solutions of (V) with their monodromy data immediately follow from our results. Under certain conditions, these solutions of $(\mathrm{V})$ admit sequences of zeros and of poles along the imaginary axis. The monodromy data are obtained by matching techniques for a perturbed linear system.
\end{abstract}

Key words: Schlesinger-type equation; fifth Painlevé equation; isomonodromy deformation; monodromy data

2010 Mathematics Subject Classification: 34M55; 34M56; 34M40; 34M35; 34E10

To the memory of Andrei A. Kapaev

\section{Introduction}

The fifth Painlevé equation normalised in the form

$$
\begin{aligned}
& \frac{\mathrm{d}^{2} y}{\mathrm{~d} x^{2}}=\left(\frac{1}{2 y}+\frac{1}{y-1}\right)\left(\frac{\mathrm{d} y}{\mathrm{~d} x}\right)^{2}-\frac{1}{x} \frac{\mathrm{d} y}{\mathrm{~d} x} \\
& +\frac{(y-1)^{2}}{8 x^{2}}\left(\left(\theta_{0}-\theta_{x}+\theta_{\infty}\right)^{2} y-\frac{\left(\theta_{0}-\theta_{x}-\theta_{\infty}\right)^{2}}{y}\right)+\left(1-\theta_{0}-\theta_{x}\right) \frac{y}{x}-\frac{y(y+1)}{2(y-1)}
\end{aligned}
$$

with $\theta_{0}, \theta_{x}, \theta_{\infty} \in \mathbb{C}$ is derived from the isomonodromy deformation of a two-dimensional linear system of the form

$$
\frac{\mathrm{d} Y}{\mathrm{~d} \lambda}=\left(\frac{A_{0}(x)}{\lambda}+\frac{A_{x}(x)}{\lambda-x}+\frac{J}{2}\right) Y
$$

with $J=\operatorname{diag}[1,-1]$ under a small change of $x$, where $A_{0}(x)$ and $A_{x}(x)$ satisfy the following:

(a) the eigenvalues of $A_{0}(x)$ and $A_{x}(x)$ are $\pm \theta_{0} / 2$ and $\pm \theta_{x} / 2$, respectively;

(b) $\left(A_{0}(x)+A_{x}(x)\right)_{11}=-\left(A_{0}(x)+A_{x}(x)\right)_{22} \equiv-\theta_{\infty} / 2$.

This paper is a contribution to the Special Issue on Painlevé Equations and Applications in Memory of Andrei Kapaev. The full collection is available at https://www.emis.de/journals/SIGMA/Kapaev.html 
Such matrices $A_{0}(x), A_{x}(x)$ may be written in the form

$$
\begin{aligned}
& A_{0}(x)=\left(\begin{array}{cc}
z+\theta_{0} / 2 & -u\left(z+\theta_{0}\right) \\
z / u & -z-\theta_{0} / 2
\end{array}\right), \\
& A_{x}(x)=\left(\begin{array}{cc}
-z-\left(\theta_{0}+\theta_{\infty}\right) / 2 & u y\left(z+\left(\theta_{0}-\theta_{x}+\theta_{\infty}\right) / 2\right) \\
-(u y)^{-1}\left(z+\left(\theta_{0}+\theta_{x}+\theta_{\infty}\right) / 2\right) & z+\left(\theta_{0}+\theta_{\infty}\right) / 2
\end{array}\right),
\end{aligned}
$$

and then

$$
y=\frac{A_{x}(x)_{12}\left(A_{0}(x)_{11}+\theta_{0} / 2\right)}{A_{0}(x)_{12}\left(A_{x}(x)_{11}+\theta_{x} / 2\right)}, \quad z=A_{0}(x)_{11}-\theta_{0} / 2, \quad u=-\frac{A_{0}(x)_{12}}{A_{0}(x)_{11}+\theta_{0} / 2}
$$

(cf. Andreev and Kitaev [2], Jimbo and Miwa [15, Appendix C]). The functions $y$ and $z$ are the same as those in $[2,15]$, and $u$ is written as $u=x^{-\theta_{\infty}} u_{\mathrm{AK}}$, where $u_{\mathrm{AK}}$ denotes the function $u$ of $[2,15]$. System (1.1) has the isomonodromy property if and only if $\left(A_{0}(x), A_{x}(x)\right)$ solves the Schlesinger-type equation

$$
x \frac{\mathrm{d} A_{0}}{\mathrm{~d} x}=\left[A_{x}, A_{0}\right], \quad x \frac{\mathrm{d} A_{x}}{\mathrm{~d} x}=\left[A_{0}, A_{x}\right]+\frac{x}{2}\left[J, A_{x}\right]
$$

(for more concrete setting of monodromy matrices for (1.1) invariant under a change of $x$, see Section 2.2); and then $y$ as in (1.2) solves (V). Conversely, for any solution $y$ of (V) there exists $\left(A_{0}(x), A_{x}(x)\right)$ satisfying (1.2) and (1.3) (cf. [14, Section 3], [15, Appendix C]).

Near $x=\infty$, two-parameter families of convergent solutions of (V) were obtained by solving the Hamiltonian system for (V) (cf. [22, 26]). Computing monodromy matrices for a system equivalent to (1.1) by WKB analysis, and using these matrices, which should be independent of $x$, Andreev and Kitaev [2] obtained asymptotic solutions of (V) near $x=0$ and $x=\infty$ on the positive real axis, and connection formulas for these solutions. Recently it was shown that, for (V) near $x=0$ (respectively, $x=+\infty$ or $x=\mathrm{i} \infty$ ), a series expression of the taufunction $\tau_{\mathrm{V}}(x)$ may be given by regular (respectively, irregular) conformal blocks (cf. Bonelli et al. [3], Gamayun et al. [9], Nagoya [20]). Furthermore, using the $s$-channel representation of the PVI tau-function and confluence procedure, Lisovyy et al. [19] gave a conjectural connection formula for $\tau_{\mathrm{V}}(x)$ between $x=0$ and $x=\mathrm{i} \infty$ [19, Conjecture $\mathrm{C}$ ] and the ratios of multipliers of $\tau_{\mathrm{V}}(x)$ as $x \rightarrow 0,+\infty, \mathrm{i} \infty[19$, Conjecture $\mathrm{D}]$.

As the first step of giving tables of critical behaviours for (V) like those of Guzzetti [12] for the sixth Painlevé equation, the author [24] presented some families of convergent solutions of $(\mathrm{V})$ near $x=0$ and the respective monodromy data parametrised by integration constants. In this paper we present a family of matrix solutions of the Schlesinger-type equation (1.3) parametrised by three integration constants $c_{0}, c_{x}, \sigma$ as $x \rightarrow \infty$ along the imaginary axis, and also the corresponding monodromy data (note that (1.3) under the restrictions (a) and (b) is regarded as a nonlinear system with respect to $(y, z, u)$ ). For the PVI Schlesinger equation and for (1.3) around $x=0$, such matrix solutions have been essentially given [23, 24]. To find solutions of (1.3) around $x=\infty$ we need quite different techniques. As explained later the monodromy data are computable by using $\left(A_{0}(x), A_{x}(x)\right)$, which is an advantage of treating solutions of (1.3) instead of those of $(\mathrm{V})$. Each entry of the solution $\left(A_{0}(x), A_{x}(x)\right)$ is a convergent series in powers of $\left(\mathrm{e}^{x} x^{\sigma-1}, \mathrm{e}^{-x} x^{-\sigma-1}\right)$ having coefficients given by asymptotic series in $x^{-1}$. This expression is valid in a sector-like domain with opening angle zero, where $\mathrm{e}^{x} x^{\sigma-1}$ and $\mathrm{e}^{-x} x^{-\sigma-1}$ are sufficiently small. This domain is larger than that known for series solutions of (V) (cf. Remark 2.20), which is another advantage of solutions of (1.3). Then we easily obtain a twoparameter family of solutions of (V) by using (1.2), whose corresponding monodromy data also follow by restricting $c_{0}$ to 1 . These monodromy data make it possible to know the parametric connection formulas between the solutions of (1.3) or (V) mentioned above and those near $x=0$ 
(respectively, those along the positive real axis near $x=\infty$ by [2]). Furthermore, by virtue of the quotient expression (1.2), under certain conditions, we may find sequences of zeros and of poles of solutions of $(\mathrm{V})$ in the sector-like domain mentioned above.

Our results are described in Section 2: families of solutions of (1.3) are given in Theorems 2.1 and 2.8; the monodromy data in Theorems 2.10, 2.11 and Corollary 2.13; families of solutions of $(\mathrm{V})$ in Theorems 2.18 and 2.21; and sequences of zeros and of poles in Theorems 2.26 and 2.27. To our goal we make an approach different from that in [2]: first construct a general solution $\left(A_{0}(x), A_{x}(x)\right)$ of (1.3) containing the integration constants $c_{0}, c_{x}, \sigma$; insert it into (1.1), which becomes a perturbed system with respect to $x^{-1}$; and finally find the monodromy matrices by matching techniques. In Section 3 , we define the families $\mathfrak{A}, \mathfrak{A}_{+}$and $\mathfrak{A}_{-}$consisting of power series in $\left(\mathrm{e}^{x} x^{\sigma-1}, \mathrm{e}^{-x} x^{-\sigma-1}\right)$, in $\mathrm{e}^{x} x^{\sigma_{0}-1}$ with $\sigma_{0}=-2 \theta_{x}-\theta_{\infty}$ and in $\mathrm{e}^{-x} x^{-\sigma_{0}^{\prime}-1}$ with $\sigma_{0}^{\prime}=2 \theta_{0}+\theta_{\infty}$, respectively, whose coefficients are asymptotic series in $x^{-1}$ in suitable sectors, and show several lemmas which are used in the construction of solutions. In Sections 4 and 5 , under the restrictions (a) and (b) we transform (1.3) into a system of integral equations, and solve it by successive approximation to obtain solutions as in Theorems 2.1 and 2.8. Section 6 is devoted to the proofs of Theorems 2.18 through 2.27 on solutions of $(\mathrm{V})$. In the final section we prove Theorems 2.10 and 2.11. Application of matchings to asymptotic solutions of the perturbed system yields monodromy matrices for (1.1) that apparently contains $x^{-1}$, and the desired matrices are obtained by letting $x \rightarrow \infty$, which is justified by the isomonodromy property. In this procedure, we use functions that are essentially WKB solutions, but for a technical reason we treat them in a method somewhat different from that in usual WKB analysis. For other Painlevé equations, WKB analysis and matching technique have been employed to find connection formulas, non-linear Stokes behaviour, distribution of poles or zeros, several examples of which are described in $[7,13]$. To this field Andrei Kapaev made pioneering contributions by using and developing the WKB matching technique in his works including [16, 17, 18]. For basic techniques of WKB analysis and related materials see [6, 21, 28].

Throughout this paper the following symbols are used.

(1) $I, J, \Delta_{+}, \Delta_{-}$denote the matrices

$$
I=\left(\begin{array}{cc}
1 & 0 \\
0 & 1
\end{array}\right), \quad J=\left(\begin{array}{cc}
1 & 0 \\
0 & -1
\end{array}\right), \quad \Delta_{+}=\left(\begin{array}{cc}
0 & 1 \\
0 & 0
\end{array}\right), \quad \Delta_{-}=\left(\begin{array}{ll}
0 & 0 \\
1 & 0
\end{array}\right) .
$$

(2) $\mathcal{R}(\mathbb{C} \backslash\{0\})$ denotes the universal covering of $\mathbb{C} \backslash\{0\}$.

(3) $\mathbb{Q}_{*}:=\mathbb{Q}\left[\theta_{0}, \theta_{x}, \theta_{\infty}, c_{0}, c_{0}^{-1}, c_{x}, c_{x}^{-1}, \sigma\right]$.

(4) For $k \in \mathbb{N} \cup\{0\},\left[x^{-k}\right]$ (respectively, $\left[x^{-k}\right]_{*}$ ) denotes a holomorphic function admitting an asymptotic representation of the form $f(x) \sim x^{-k} \sum_{j \geq 0} f_{j} x^{-j}$ with $f_{j} \in \mathbb{Q}_{*}$ (respectively, $f_{j} \in \mathbb{Q}\left(\theta_{0}, \theta_{x}, \theta_{\infty}, c, \sigma\right)$ with $\left.c=\left(c^{\prime}\right)^{-1}=c_{x} / c_{0}\right)$ as $x \rightarrow \infty$ through a specified sector, each $f_{j}$ not being necessarily nonzero (e.g., note that $\left[x^{-k}\right]$ with $k \in \mathbb{N}$ may also be denoted by $\left.[1]\left(=\left[x^{0}\right]\right)\right)$. Furthermore in Sections 4 and 5 , for simplicity, we often denote by $(1)_{x}$ a function given by $1+\left[x^{-1}\right]$.

(5) Sectors and domains:

$$
\begin{aligned}
& \Sigma\left(\sigma, \varepsilon, x_{\infty}, \delta\right):|\arg x-\pi / 2|<\pi / 2-\delta, \quad\left|\mathrm{e}^{x} x^{\sigma-1}\right|<\varepsilon, \quad\left|\mathrm{e}^{-x} x^{-\sigma-1}\right|<\varepsilon, \quad|x|>x_{\infty}, \\
& D\left(B_{*}, \varepsilon, x_{\infty}, \delta\right)=\bigcup_{\sigma \in B_{*}}\{\sigma\} \times \Sigma\left(\sigma, \varepsilon, x_{\infty}, \delta\right), \\
& \Sigma_{*}\left(\varepsilon, x_{\infty}, \delta\right): \quad-(\pi / 2-\delta)<\arg x-\pi / 2<\pi-\delta, \quad\left|\mathrm{e}^{x} x^{\sigma_{0}-1}\right|<\varepsilon, \quad|x|>x_{\infty}, \\
& \Sigma_{*}^{\prime}\left(\varepsilon, x_{\infty}, \delta\right): \quad-(\pi-\delta)<\arg x-\pi / 2<\pi / 2-\delta, \quad\left|\mathrm{e}^{-x} x^{-\sigma_{0}^{\prime}-1}\right|<\varepsilon, \quad|x|>x_{\infty},
\end{aligned}
$$




$$
\begin{aligned}
& \Sigma_{* *}\left(x_{\infty}, \delta\right):|\arg x-\pi / 2|<\pi-\delta, \quad|x|>x_{\infty}, \\
& \Sigma_{0}\left(x_{\infty}, \delta\right):|\arg x-\pi / 2|<\pi / 2-\delta, \quad|x|>x_{\infty}, \\
& \Sigma_{\pi}\left(\Theta_{1}, \Theta_{2} ; x_{\infty}\right): \quad(\pi / 2<) \Theta_{1}<\arg x<\Theta_{2}(<3 \pi / 2), \quad|x|>x_{\infty} .
\end{aligned}
$$

(6) For the integration constants $c_{0}, c_{x}$ and $\sigma$, we frequently write

$$
\begin{array}{ll}
\gamma_{+}^{0}:=c_{0}\left(\sigma+2 \theta_{0}-\theta_{\infty}\right) / 4, & \gamma_{-}^{0}:=c_{0}^{-1}\left(-\sigma+2 \theta_{0}+\theta_{\infty}\right) / 4, \\
\gamma_{+}^{x}:=c_{x}\left(-\sigma+2 \theta_{x}-\theta_{\infty}\right) / 4, & \gamma_{-}^{x}:=c_{x}^{-1}\left(\sigma+2 \theta_{x}+\theta_{\infty}\right) / 4, \\
\mathbf{c}:=\left(c_{0}, c_{x}\right), \quad c:=c_{x} / c_{0}, & c^{\prime}:=c_{0} / c_{x} .
\end{array}
$$

(7) For a sequence $\left\{\phi^{j}\right\}, \triangle \phi^{j}:=\phi^{j}-\phi^{j-1}$ in Section 5 .

\section{Results}

\subsection{Solutions of the Schlesinger-type equation}

For $\delta, \varepsilon, x_{\infty}$ satisfying $\delta<\pi / 2, x_{\infty}>\varepsilon^{-1}$, and for each $\sigma \in B_{*} \subset \mathbb{C}$, define $\Sigma\left(\sigma, \varepsilon, x_{\infty}, \delta\right) \subset$ $\mathcal{R}(\mathbb{C} \backslash\{0\})$ by

$$
\Sigma\left(\sigma, \varepsilon, x_{\infty}, \delta\right):|\arg x-\pi / 2|<\pi / 2-\delta, \quad\left|\mathrm{e}^{x} x^{\sigma-1}\right|<\varepsilon, \quad\left|\mathrm{e}^{-x} x^{-\sigma-1}\right|<\varepsilon, \quad|x|>x_{\infty}
$$

and $D\left(B_{*}, \varepsilon, x_{\infty}, \delta\right) \subset B_{*} \times \mathcal{R}(\mathbb{C} \backslash\{0\}) \subset \mathbb{C} \times \mathcal{R}(\mathbb{C} \backslash\{0\})$ by

$$
D\left(B_{*}, \varepsilon, x_{\infty}, \delta\right):=\bigcup_{\sigma \in B_{*}}\{\sigma\} \times \Sigma\left(\sigma, \varepsilon, x_{\infty}, \delta\right) .
$$

Theorem 2.1. Let $B_{*} \subset \mathbb{C}$ and $B_{0}, B_{x} \subset \mathbb{C} \backslash\{0\}$ be given bounded domains, and let $\delta$ be a given positive number such that $\delta<\pi / 2$. Then equation (1.3) admits a three-parameter family of solutions

$$
\left\{\left(A_{0}(\mathbf{c}, \sigma, x), A_{x}(\mathbf{c}, \sigma, x)\right) ;(\mathbf{c}, \sigma):=\left(c_{0}, c_{x}, \sigma\right) \in B_{0} \times B_{x} \times B_{*}\right\}
$$

with

$$
\begin{aligned}
& A_{0}(\mathbf{c}, \sigma, x)=f_{0}(\mathbf{c}, \sigma, x) J+f_{+}(\mathbf{c}, \sigma, x) \Delta_{+}+f_{-}(\mathbf{c}, \sigma, x) \Delta_{-}, \\
& A_{x}(\mathbf{c}, \sigma, x)=g_{0}(\mathbf{c}, \sigma, x) J+g_{+}(\mathbf{c}, \sigma, x) \Delta_{+}+g_{-}(\mathbf{c}, \sigma, x) \Delta_{-}
\end{aligned}
$$

satisfying the conditions (a) and (b). The entries are holomorphic in $(\mathbf{c}, \sigma, x) \in B_{0} \times B_{x} \times$ $D\left(B_{*}, \varepsilon, x_{\infty}, \delta\right)$, and are represented by the convergent series in powers of $\left(\mathrm{e}^{x} x^{\sigma-1}, \mathrm{e}^{-x} x^{-\sigma-1}\right)$ as follows:

$$
\begin{aligned}
& f_{0}(\mathbf{c}, \sigma, x)=\left(\sigma-\theta_{\infty}\right) / 4-\left(\left(\sigma+\theta_{\infty}\right) \gamma_{+}^{0} \gamma_{-}^{0}+\left(\sigma-\theta_{\infty}\right) \gamma_{+}^{x} \gamma_{-}^{x}\right) x^{-2} / 2+\left[x^{-3}\right] \\
& +\gamma_{-}^{0} \gamma_{+}^{x}\left(1+\left[x^{-1}\right]\right) \mathrm{e}^{x} x^{\sigma-1}+\sum_{n=2}^{\infty}\left(\gamma_{-}^{0} \gamma_{+}^{x}\right)^{n}\left[x^{-n+1}\right]\left(\mathrm{e}^{x} x^{\sigma-1}\right)^{n} \\
& +\gamma_{+}^{0} \gamma_{-}^{x}\left(1+\left[x^{-1}\right]\right) \mathrm{e}^{-x} x^{-\sigma-1}+\sum_{n=2}^{\infty}\left(\gamma_{+}^{0} \gamma_{-}^{x}\right)^{n}\left[x^{-n+1}\right]\left(\mathrm{e}^{-x} x^{-\sigma-1}\right)^{n}, \\
& g_{0}(\mathbf{c}, \sigma, x)=-\theta_{\infty} / 2-f_{0}(\mathbf{c}, \sigma, x), \\
& x^{\left(\sigma+\theta_{\infty}\right) / 2} f_{+}(\mathbf{c}, \sigma, x)=\gamma_{+}^{0}\left(1+\left[x^{-1}\right]\right)-\gamma_{+}^{x}\left(\left(\sigma-\theta_{\infty}\right) / 2+\left[x^{-1}\right]\right) \mathrm{e}^{x} x^{\sigma-1} \\
& -\gamma_{-}^{0}\left(\gamma_{+}^{x}\right)^{2}\left(1+\left[x^{-1}\right]\right)\left(\mathrm{e}^{x} x^{\sigma-1}\right)^{2}+\sum_{n=3}^{\infty} \gamma_{+}^{x}\left(\gamma_{-}^{0} \gamma_{+}^{x}\right)^{n-1}\left[x^{-n+2}\right]\left(\mathrm{e}^{x} x^{\sigma-1}\right)^{n}
\end{aligned}
$$




$$
\begin{aligned}
& +2\left(\gamma_{+}^{0}\right)^{2} \gamma_{-}^{x}\left(1+\left[x^{-1}\right]\right) \mathrm{e}^{-x} x^{-\sigma-2}+\sum_{n=2}^{\infty} \gamma_{+}^{0}\left(\gamma_{+}^{0} \gamma_{-}^{x}\right)^{n}\left[x^{-n}\right]\left(\mathrm{e}^{-x} x^{-\sigma-1}\right)^{n}, \\
& \mathrm{e}^{-x} x^{-\left(\sigma-\theta_{\infty}\right) / 2} g_{+}(\mathbf{c}, \sigma, x)=\gamma_{+}^{x}\left(1+\left[x^{-1}\right]\right)+2 \gamma_{-}^{0}\left(\gamma_{+}^{x}\right)^{2}\left(1+\left[x^{-1}\right]\right) \mathrm{e}^{x} x^{\sigma-2} \\
& +\sum_{n=2}^{\infty} \gamma_{+}^{x}\left(\gamma_{-}^{0} \gamma_{+}^{x}\right)^{n}\left[x^{-n}\right]\left(\mathrm{e}^{x} x^{\sigma-1}\right)^{n}-\gamma_{+}^{0}\left(\left(\sigma+\theta_{\infty}\right) / 2+\left[x^{-1}\right]\right) \mathrm{e}^{-x} x^{-\sigma-1} \\
& -\left(\gamma_{+}^{0}\right)^{2} \gamma_{-}^{x}\left(1+\left[x^{-1}\right]\right)\left(\mathrm{e}^{-x} x^{-\sigma-1}\right)^{2}+\sum_{n=3}^{\infty} \gamma_{+}^{0}\left(\gamma_{+}^{0} \gamma_{-}^{x}\right)^{n-1}\left[x^{-n+2}\right]\left(\mathrm{e}^{-x} x^{-\sigma-1}\right)^{n}, \\
& x^{-\left(\sigma+\theta_{\infty}\right) / 2} f_{-}(\mathbf{c}, \sigma, x)=\gamma_{-}^{0}\left(1+\left[x^{-1}\right]\right)+2\left(\gamma_{-}^{0}\right)^{2} \gamma_{+}^{x}\left(1+\left[x^{-1}\right]\right) \mathrm{e}^{x} x^{\sigma-2} \\
& +\sum_{n=2}^{\infty} \gamma_{-}^{0}\left(\gamma_{-}^{0} \gamma_{+}^{x}\right)^{n}\left[x^{-n}\right]\left(\mathrm{e}^{x} x^{\sigma-1}\right)^{n}-\gamma_{-}^{x}\left(\left(\sigma-\theta_{\infty}\right) / 2+\left[x^{-1}\right]\right) \mathrm{e}^{-x} x^{-\sigma-1} \\
& -\gamma_{+}^{0}\left(\gamma_{-}^{x}\right)^{2}\left(1+\left[x^{-1}\right]\right)\left(\mathrm{e}^{-x} x^{-\sigma-1}\right)^{2}+\sum_{n=3}^{\infty} \gamma_{-}^{x}\left(\gamma_{+}^{0} \gamma_{-}^{x}\right)^{n-1}\left[x^{-n+2}\right]\left(\mathrm{e}^{-x} x^{-\sigma-1}\right)^{n}, \\
& \mathrm{e}^{x} x^{\left(\sigma-\theta_{\infty}\right) / 2} g_{-}(\mathbf{c}, \sigma, x)=\gamma_{-}^{x}\left(1+\left[x^{-1}\right]\right)-\gamma_{-}^{0}\left(\left(\sigma+\theta_{\infty}\right) / 2+\left[x^{-1}\right]\right) \mathrm{e}^{x} x^{\sigma-1} \\
& -\left(\gamma_{-}^{0}\right)^{2} \gamma_{+}^{x}\left(1+\left[x^{-1}\right]\right)\left(\mathrm{e}^{x} x^{\sigma-1}\right)^{2}+\sum_{n=3}^{\infty} \gamma_{-}^{0}\left(\gamma_{-}^{0} \gamma_{+}^{x}\right)^{n-1}\left[x^{-n+2}\right]\left(\mathrm{e}^{x} x^{\sigma-1}\right)^{n} \\
& +2 \gamma_{+}^{0}\left(\gamma_{-}^{x}\right)^{2}\left(1+\left[x^{-1}\right]\right) \mathrm{e}^{-x} x^{-\sigma-2}+\sum_{n=2}^{\infty} \gamma_{-}^{x}\left(\gamma_{+}^{0} \gamma_{-}^{x}\right)^{n}\left[x^{-n}\right]\left(\mathrm{e}^{-x} x^{-\sigma-1}\right)^{n}
\end{aligned}
$$

Here

(i) $\varepsilon=\varepsilon\left(B_{0}, B_{x}, B_{*}, \delta\right)$ (respectively, $\left.x_{\infty}=x_{\infty}\left(B_{0}, B_{x}, B_{*}, \delta\right)>\varepsilon^{-1}\right)$ is a sufficiently small (respectively, large) positive number depending on $\left(B_{0}, B_{x}, B_{*}, \delta\right)$;

(ii) $\gamma_{ \pm}^{0}=\gamma_{ \pm}^{0}(\mathbf{c}, \sigma), \gamma_{ \pm}^{x}=\gamma_{ \pm}^{x}(\mathbf{c}, \sigma)$ denote

$$
\begin{aligned}
& \gamma_{+}^{0}=c_{0}\left(\sigma+2 \theta_{0}-\theta_{\infty}\right) / 4, \quad \gamma_{-}^{0}=c_{0}^{-1}\left(-\sigma+2 \theta_{0}+\theta_{\infty}\right) / 4, \\
& \gamma_{+}^{x}=c_{x}\left(-\sigma+2 \theta_{x}-\theta_{\infty}\right) / 4, \quad \gamma_{-}^{x}=c_{x}^{-1}\left(\sigma+2 \theta_{x}+\theta_{\infty}\right) / 4 ;
\end{aligned}
$$

(iii) the asymptotic series for $\left[x^{-1}\right],\left[x^{-n}\right], \ldots$ are valid uniformly in $(\mathbf{c}, \sigma) \in B_{0} \times B_{x} \times B_{*}$ as $x$ tends to $\infty$ through the sector $|\arg x-\pi / 2|<\pi / 2-\delta,|x|>x_{\infty}$.

Remark 2.2. The restriction (a) implies the relations $f_{0}(\mathbf{c}, \sigma, x)^{2}+f_{+}(\mathbf{c}, \sigma, x) f_{-}(\mathbf{c}, \sigma, x) \equiv \theta_{0}^{2} / 4$ and $g_{0}(\mathbf{c}, \sigma, x)^{2}+g_{+}(\mathbf{c}, \sigma, x) g_{-}(\mathbf{c}, \sigma, x) \equiv \theta_{x}^{2} / 4$.

Remark 2.3. More precisely, $\varepsilon=\varepsilon\left(B_{0}, B_{x}, B_{*}, \delta\right)$ may be chosen in such a way that

$$
\left(\left|\gamma_{-}^{0} \gamma_{+}^{x}\right|+\left|\gamma_{+}^{0} \gamma_{-}^{x}\right|+\left|\gamma_{+}^{0}\right|+\left|\gamma_{-}^{0}\right|+\left|\gamma_{+}^{x}\right|+\left|\gamma_{-}^{x}\right|+1\right)\left(\left|\gamma_{+}^{0}\right|+\left|\gamma_{-}^{0}\right|+\left|\gamma_{+}^{x}\right|+\left|\gamma_{-}^{x}\right|+1\right) \varepsilon \leq r_{0}(\delta)
$$

for every $\left(c_{0}, c_{x}, \sigma\right) \in B_{0} \times B_{x} \times B_{*}$, where $r_{0}(\delta)<1$ is a sufficiently small positive number depending on $\delta$ (see Sections 5.2, 5.4 and Proposition 5.3).

Remark 2.4. The sector-like domain $\Sigma\left(\sigma, \varepsilon, x_{\infty}, \delta\right)$ is given by $|x|>x_{\infty}$ and

$$
\begin{aligned}
-(1+\operatorname{Re} \sigma) \log |x|+\operatorname{Im} \sigma \cdot \arg x+\log \left(\varepsilon^{-1}\right) \\
\quad<\operatorname{Re} x<(1-\operatorname{Re} \sigma) \log |x|+\operatorname{Im} \sigma \cdot \arg x-\log \left(\varepsilon^{-1}\right),
\end{aligned}
$$

where $\operatorname{Im} \sigma \cdot \arg x=O(1)$ since $|\arg x-\pi / 2|<\pi / 2-\delta$ (cf. Fig. 2.1). 


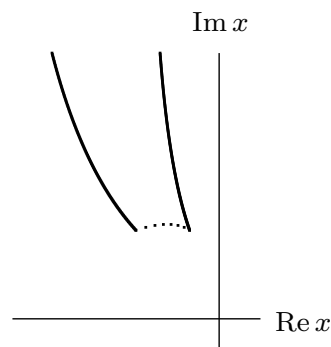

(a) $\operatorname{Re} \sigma>1$

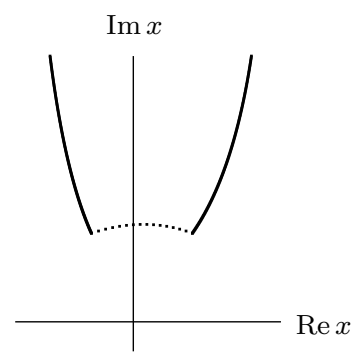

(b) $|\operatorname{Re} \sigma|<1$

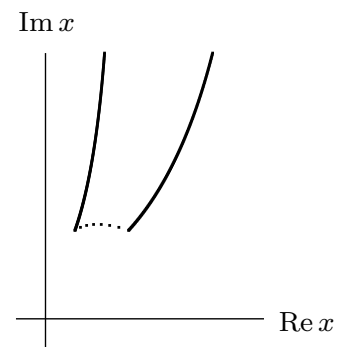

(c) $\operatorname{Re} \sigma<-1$

Figure 2.1. $\Sigma\left(\sigma, \varepsilon, x_{\infty}, \delta\right)$.

Remark 2.5. The asymptotic series for $\left[x^{-1}\right],\left[x^{-n}\right], \ldots$ seem to be valid in an extended sector $|\arg x-\pi / 2|<\pi-\delta$ (cf. [22, 26]).

Remark 2.6. For $k \in \mathbb{Z}$, let $\Sigma_{k}\left(\sigma, \varepsilon, x_{\infty}, \delta\right) \subset \mathcal{R}(\mathbb{C} \backslash\{0\})$ be the sector-like domain defined by

$$
|\arg x-(1 / 2+k) \pi|<\pi / 2-\delta, \quad\left|\mathrm{e}^{x} x^{\sigma-1}\right|<\varepsilon, \quad\left|\mathrm{e}^{-x} x^{-\sigma-1}\right|<\varepsilon, \quad|x|>x_{\infty}
$$

(note that $\Sigma_{0}\left(\sigma, \varepsilon, x_{\infty}, \delta\right)=\Sigma\left(\sigma, \varepsilon, x_{\infty}, \delta\right)$ ). Then in the domain $D_{k}\left(B_{*}, \varepsilon, x_{\infty}, \delta\right):=\bigcup_{\sigma \in B_{*}}\{\sigma\} \times$ $\Sigma_{k}\left(\sigma, \varepsilon, x_{\infty}, \delta\right)$ equation (1.3) admits a family of solutions $\left\{\left(A_{0}^{(k)}(\mathbf{c}, \sigma, x), A_{x}^{(k)}(\mathbf{c}, \sigma, x)\right)\right\}$ having an expression of the same form as in Theorem 2.1 with $\left[x^{-1}\right],\left[x^{-n}\right], \ldots$ in the sector $\mid \arg x-$ $(1 / 2+k) \pi \mid<\pi / 2-\delta$.

Remark 2.7. By Theorem 2.1 and Remark 5.6 the tau-function is given by

$$
\begin{aligned}
\frac{\mathrm{d}}{\mathrm{d} x} \log \tau_{\mathrm{V}}(x)= & x^{-1} \operatorname{tr}\left(A_{0} A_{x}\right)-\operatorname{tr}\left(A_{0} J / 2\right)-\theta_{\infty} / 2 \\
= & \left(A_{x}\right)_{11}+x^{-1}\left(2\left(A_{0}\right)_{11}\left(A_{x}\right)_{11}+\left(A_{0}\right)_{12}\left(A_{x}\right)_{21}+\left(A_{0}\right)_{21}\left(A_{x}\right)_{12}\right) \\
= & -\left(\sigma+\theta_{\infty}\right) / 4-\left(\sigma^{2}-\theta_{\infty}^{2}\right) x^{-1} / 8 \\
& -\left(\left(\sigma+\theta_{\infty}\right) \gamma_{+}^{0} \gamma_{-}^{0}+\left(\sigma-\theta_{\infty}\right) \gamma_{+}^{x} \gamma_{-}^{x}\right) x^{-2} / 2+\left[x^{-3}\right] \\
& -\gamma_{-}^{0} \gamma_{+}^{x}\left(1+\left[x^{-1}\right]\right) \mathrm{e}^{x} x^{\sigma-2}+\gamma_{+}^{0} \gamma_{-}^{x}\left(1+\left[x^{-1}\right]\right) \mathrm{e}^{-x} x^{-\sigma-2} \\
& +\sum_{n=2}^{\infty}\left(\gamma_{-}^{0} \gamma_{+}^{x}\right)^{n}\left[x^{-n+1}\right]\left(\mathrm{e}^{x} x^{\sigma-1}\right)^{n}+\sum_{n=2}^{\infty}\left(\gamma_{+}^{0} \gamma_{-}^{x}\right)^{n}\left[x^{-n+1}\right]\left(\mathrm{e}^{-x} x^{-\sigma-1}\right)^{n} .
\end{aligned}
$$

It may be checked that first some terms agree with those of $\tau(t \rightarrow \mathrm{i} \infty)$ in [19, equation (1.12a)]. It was conjectured by $[3,20]$ that $\tau_{\mathrm{V}}(x)$ is represented by an infinite sum of irregular conformal blocks, whose full structure may be observed explicitly. Conformal field theory (with the Fredholm determinant) yields such expansions not via $(\mathrm{d} / \mathrm{d} x) \log \tau_{\mathrm{V}}(x)$. On the other hand, from the fourth-order bilinear equation $[2,14,15]$

$$
\begin{aligned}
& x^{3}\left(\tau \tau^{(4)}-4 \tau^{\prime} \tau^{(3)}+3\left(\tau^{\prime \prime}\right)^{2}\right)+4 x^{2}\left(\tau \tau^{(3)}-\tau^{\prime} \tau^{\prime \prime}\right)-\left(x^{2}-2 \theta_{\infty} x+\theta_{0}^{2}+\theta_{x}^{2}\right) x\left(\tau \tau^{\prime \prime}-\left(\tau^{\prime}\right)^{2}\right) \\
& +2 x \tau \tau^{\prime \prime}+\left(\theta_{\infty} x-\theta_{0}^{2}-\theta_{x}^{2}\right) \tau \tau^{\prime}-\theta_{x}^{2} \theta_{\infty} \tau^{2} / 2=0
\end{aligned}
$$

which does not apparently contain the logarithmic derivative, first some terms of $\tau_{\mathrm{V}}(x)$ may be obtained by an argument similar to that in [8, Section 3] for $\tau_{\mathrm{VI}}(x)$. It seems difficult to derive the full expansion without finding a suitable structure of this equation.

For special values of $\sigma, c_{0}, c_{x}$, we have a two-parameter or one-parameter family of solutions. If $\sigma=-2 \theta_{x}-\theta_{\infty}$, namely, $\gamma_{-}^{x}=0$, we have 
Theorem 2.8. Suppose that $\theta_{x} \neq 0$ and $\sigma=\sigma_{0}:=-2 \theta_{x}-\theta_{\infty}$. Let $\Sigma_{*}\left(\varepsilon, x_{\infty}, \delta\right) \subset \mathcal{R}(\mathbb{C} \backslash\{0\})$ be the domain defined by

$$
\Sigma_{*}\left(\varepsilon, x_{\infty}, \delta\right): \quad-(\pi / 2-\delta)<\arg x-\pi / 2<\pi-\delta, \quad\left|\mathrm{e}^{x} x^{\sigma_{0}-1}\right|<\varepsilon, \quad|x|>x_{\infty},
$$

where $\delta<\pi / 2$ is a given positive number. Let $\tilde{B} \subset \mathbb{C}$ be a given bounded domain, and $B_{0}$ as in Theorem 2.1. Then equation (1.3) admits a two-parameter family of solutions

$$
\left\{\left(A_{0}(\mathbf{c}, x), A_{x}(\mathbf{c}, x)\right) ; \mathbf{c}=\left(c_{0}, c_{x}\right) \in B_{0} \times \tilde{B}\right\}
$$

with

$$
\begin{aligned}
& A_{0}(\mathbf{c}, x)=f_{0}(\mathbf{c}, x) J+f_{+}(\mathbf{c}, x) \Delta_{+}+f_{-}(\mathbf{c}, x) \Delta_{-}, \\
& A_{x}(\mathbf{c}, x)=g_{0}(\mathbf{c}, x) J+g_{+}(\mathbf{c}, x) \Delta_{+}+g_{-}(\mathbf{c}, x) \Delta_{-}
\end{aligned}
$$

such that the entries are holomorphic in $(\mathbf{c}, x) \in B_{0} \times \tilde{B} \times \Sigma_{*}\left(\varepsilon, x_{\infty}, \delta\right)$ and are represented by the convergent series in powers of $\mathrm{e}^{x} x^{\sigma_{0}-1}$ as follows:

$$
\begin{aligned}
& f_{0}(\mathbf{c}, x)=-\left(\theta_{x}+\theta_{\infty}\right) / 2+\theta_{x} \gamma_{+*}^{0} \gamma_{-*}^{0} x^{-2}+\left[x^{-3}\right] \\
& +\gamma_{-*}^{0} \gamma_{+*}^{x}\left(1+\left[x^{-1}\right]\right) \mathrm{e}^{x} x^{\sigma_{0}-1}+\sum_{n=2}^{\infty}\left(\gamma_{-*}^{0} \gamma_{+*}^{x}\right)^{n}\left[x^{-n+1}\right]\left(\mathrm{e}^{x} x^{\sigma_{0}-1}\right)^{n}, \\
& g_{0}(\mathbf{c}, x)=-\theta_{\infty} / 2-f_{0}(\mathbf{c}, x) \text {, } \\
& x^{-\theta_{x}} f_{+}(\mathbf{c}, x)=\gamma_{+*}^{0}\left(1+\left[x^{-1}\right]\right)+\gamma_{+*}^{x}\left(\theta_{x}+\theta_{\infty}+\left[x^{-1}\right]\right) \mathrm{e}^{x} x^{\sigma_{0}-1} \\
& -\gamma_{-*}^{0}\left(\gamma_{+*}^{x}\right)^{2}\left(1+\left[x^{-1}\right]\right)\left(\mathrm{e}^{x} x^{\sigma_{0}-1}\right)^{2}+\sum_{n=3}^{\infty} \gamma_{+*}^{x}\left(\gamma_{-*}^{0} \gamma_{+*}^{x}\right)^{n-1}\left[x^{-n+2}\right]\left(\mathrm{e}^{x} x^{\sigma_{0}-1}\right)^{n}, \\
& \mathrm{e}^{-x} x^{\theta_{x}+\theta_{\infty}} g_{+}(\mathbf{c}, x)=\gamma_{+*}^{x}\left(1+\left[x^{-1}\right]\right)+2 \gamma_{-*}^{0}\left(\gamma_{+*}^{x}\right)^{2}\left(1+\left[x^{-1}\right]\right) \mathrm{e}^{x} x^{\sigma_{0}-2} \\
& +\sum_{n=2}^{\infty} \gamma_{+*}^{x}\left(\gamma_{-*}^{0} \gamma_{+*}^{x}\right)^{n}\left[x^{-n}\right]\left(\mathrm{e}^{x} x^{\sigma_{0}-1}\right)^{n}+\gamma_{+*}^{0}\left(\theta_{x}+\left[x^{-1}\right]\right) \mathrm{e}^{-x} x^{-\sigma_{0}-1}, \\
& x^{\theta_{x}} f_{-}(\mathbf{c}, x)=\gamma_{-*}^{0}\left(1+\left[x^{-1}\right]\right)+2\left(\gamma_{-*}^{0}\right)^{2} \gamma_{+*}^{x}\left(1+\left[x^{-1}\right]\right) \mathrm{e}^{x} x^{\sigma_{0}-2} \\
& +\sum_{n=2}^{\infty} \gamma_{-*}^{0}\left(\gamma_{-*}^{0} \gamma_{+*}^{x}\right)^{n}\left[x^{-n}\right]\left(\mathrm{e}^{x} x^{\sigma_{0}-1}\right)^{n}, \\
& \mathrm{e}^{x} x^{-\theta_{x}-\theta_{\infty}} g_{-}(\mathbf{c}, x)=\gamma_{-*}^{0}\left(\theta_{x}+\left[x^{-1}\right]\right) \mathrm{e}^{x} x^{\sigma_{0}-1}-\left(\gamma_{-*}^{0}\right)^{2} \gamma_{+*}^{x}\left(1+\left[x^{-1}\right]\right)\left(\mathrm{e}^{x} x^{\sigma_{0}-1}\right)^{2} \\
& +\sum_{n=3}^{\infty} \gamma_{-*}^{0}\left(\gamma_{-*}^{0} \gamma_{+*}^{x}\right)^{n-1}\left[x^{-n+2}\right]\left(\mathrm{e}^{x} x^{\sigma_{0}-1}\right)^{n}
\end{aligned}
$$

Here

(i) $\varepsilon=\varepsilon\left(B_{0}, \tilde{B}, \delta\right)$ (respectively, $x_{\infty}=x_{\infty}\left(B_{0}, \tilde{B}, \delta\right)$ ) is a sufficiently small (respectively, large) positive number depending on $\left(B_{0}, \tilde{B}, \delta\right)$;

(ii) $\gamma_{ \pm *}^{0}:=\gamma_{ \pm}^{0}\left(\mathbf{c}, \sigma_{0}\right), \gamma_{+*}^{x}:=\gamma_{+}^{x}\left(\mathbf{c}, \sigma_{0}\right)$, that is,

$$
\gamma_{+*}^{0}=c_{0}\left(\theta_{0}-\theta_{x}-\theta_{\infty}\right) / 2, \quad \gamma_{-*}^{0}=c_{0}^{-1}\left(\theta_{0}+\theta_{x}+\theta_{\infty}\right) / 2, \quad \gamma_{+*}^{x}=c_{x} \theta_{x} ;
$$

(iii) the asymptotic series for $\left[x^{-1}\right],\left[x^{-n}\right], \ldots$ are valid uniformly in $\mathbf{c} \in B_{0} \times \tilde{B}$ as $x$ tends to $\infty$ through the sector $-(\pi / 2-\delta)<\arg x-\pi / 2<\pi-\delta,|x|>x_{\infty}$, and the coefficients of the series are in $\mathbb{Q}\left[\theta_{0}, \theta_{x}, \theta_{\infty}, c_{0}, c_{0}^{-1}, c_{x}\right] \subset \mathbb{Q}_{*}$. 
In addition to $\sigma=\sigma_{0}$, if $c_{x}=0$, then (1.3) admits a one-parameter family of solutions

$$
\left\{\left(A_{0}\left(c_{0}, x\right), A_{x}\left(c_{0}, x\right)\right) ; c_{0} \in B_{0}\right\}
$$

with

$$
\begin{aligned}
& A_{0}\left(c_{0}, x\right)=f_{0}\left(c_{0}, x\right) J+f_{+}\left(c_{0}, x\right) \Delta_{+}+f_{-}\left(c_{0}, x\right) \Delta_{-}, \\
& A_{x}\left(c_{0}, x\right)=g_{0}\left(c_{0}, x\right) J+g_{+}\left(c_{0}, x\right) \Delta_{+}+g_{-}\left(c_{0}, x\right) \Delta_{-},
\end{aligned}
$$

whose entries are holomorphic in $\left(c_{0}, x\right) \in B_{0} \times \Sigma_{* *}\left(x_{\infty}, \delta\right)$ with $\Sigma_{* *}\left(x_{\infty}, \delta\right):|\arg x-\pi / 2|<\pi-\delta$, $|x|>x_{\infty}$ for some $x_{\infty}=x_{\infty}\left(B_{0}, \delta\right)$, and are represented by the asymptotic series

$$
\begin{aligned}
& f_{0}\left(c_{0}, x\right)=-\left(\theta_{x}+\theta_{\infty}\right) / 2+\theta_{x}\left(\theta_{0}^{2}-\left(\theta_{x}+\theta_{\infty}\right)^{2}\right) x^{-2} / 4+\left[x^{-3}\right], \\
& g_{0}\left(c_{0}, x\right)=-\theta_{\infty} / 2-f_{0}\left(c_{0}, x\right), \\
& x^{-\theta_{x}} f_{+}\left(c_{0}, x\right)=c_{0}\left(\theta_{0}-\theta_{x}-\theta_{\infty}\right)\left(1 / 2+\left[x^{-1}\right]\right), \\
& x^{-\theta_{x}+1} g_{+}\left(c_{0}, x\right)=c_{0}\left(\theta_{0}-\theta_{x}-\theta_{\infty}\right)\left(\theta_{x} / 2+\left[x^{-1}\right]\right), \\
& x^{\theta_{x}} f_{-}\left(c_{0}, x\right)=c_{0}^{-1}\left(\theta_{0}+\theta_{x}+\theta_{\infty}\right)\left(1 / 2+\left[x^{-1}\right]\right), \\
& x^{\theta_{x}+1} g_{-}\left(c_{0}, x\right)=c_{0}^{-1}\left(\theta_{0}+\theta_{x}+\theta_{\infty}\right)\left(\theta_{x} / 2+\left[x^{-1}\right]\right),
\end{aligned}
$$

uniformly in $c_{0} \in B_{0}$ as $x$ tends to $\infty$ through $\Sigma_{* *}\left(x_{\infty}, \delta\right)$, the coefficients of $\left[x^{-1}\right], \ldots$ being in $\mathbb{Q}\left[\theta_{0}, \theta_{x}, \theta_{\infty}, c_{0}, c_{0}^{-1}\right]$.

Remark 2.9. If we put $\sigma=\sigma_{0}^{\prime}=2 \theta_{0}+\theta_{\infty}$ under the supposition $\theta_{0} \neq 0$, we get a two-parameter family of solutions represented by a power series in $\mathrm{e}^{-x} x^{-\sigma_{0}^{\prime}-1}$ in the domain $\Sigma_{*}^{\prime}\left(\varepsilon, x_{\infty}, \delta\right)$ : $-(\pi-\delta)<\arg x-\pi / 2<\pi / 2-\delta,\left|\mathrm{e}^{-x} x^{-\sigma_{0}^{\prime}-1}\right|<\varepsilon,|x|>x_{\infty}$. If $\sigma=2 \theta_{x}-\theta_{\infty}, \theta_{x} \neq 0$ (respectively, $\left.\sigma=-2 \theta_{0}+\theta_{\infty}, \theta_{0} \neq 0\right)$, then there exist solutions expanded into series in $\mathrm{e}^{-x} x^{-2 \theta_{x}+\theta_{\infty}-1}$ (respectively, $\mathrm{e}^{x} x^{-2 \theta_{0}+\theta_{\infty}-1}$ ).

\subsection{Monodromy data}

System (1.1) with (a) and (b) admits a fundamental matrix solution of the form

$$
Y(x, \lambda)=\left(I+O\left(\lambda^{-1}\right)\right) \mathrm{e}^{(\lambda / 2) J} \lambda^{-\left(\theta_{\infty} / 2\right) J}
$$

as $\lambda \rightarrow \infty$ through the sector $-\pi / 2<\arg \lambda<3 \pi / 2$. Denote by $Y_{1}(x, \lambda)$ and $Y_{2}(x, \lambda)$ the matrix solutions having asymptotic representations of the same form as in (2.1) in the sectors $-3 \pi / 2<\arg \lambda<\pi / 2$ and $\pi / 2<\arg \lambda<5 \pi / 2$, respectively. In accordance with [2, Section 2], [24, Section 2.4] let $S_{1}=I+s_{1} \Delta_{-}$and $S_{2}=I+s_{2} \Delta_{+}$be the Stokes multipliers given by

$$
Y(x, \lambda)=Y_{1}(x, \lambda) S_{1}, \quad Y_{2}(x, \lambda)=Y(x, \lambda) S_{2},
$$

and let $M_{0}, M_{x}, M_{\infty} \in \mathrm{SL}_{2}(\mathbb{C})$ be the monodromy matrices defined by the analytic continuation of $Y(x, \lambda)$ along loops $l_{0}, l_{x}, l_{\infty} \in \pi_{1}\left(P^{1}(\mathbb{C}) \backslash\{0, x, \infty\}\right)$ located as in Fig. 2.2 for $x$ such that $-\pi<\arg x<\pi$. They surround, respectively, $\lambda=0, x, \infty$ in the positive sense and satisfy $l_{0} l_{x} l_{\infty}=\mathrm{id}$, which implies $M_{\infty} M_{x} M_{0}=I$.

System (1.1) has the isomonodromy property, that is, the matrices $M_{0}, M_{x}, S_{1}, S_{2}$ are invariant under the change of $x$ if and only if $\left(A_{0}, A_{x}\right)$ solves (1.3). Then each solution of (1.3) corresponds to some $\left(M_{0}, M_{x}\right) \in \mathrm{SL}_{2}(\mathbb{C})^{2}$ not depending on $x$, and then, by

$$
M_{\infty}=M_{0}^{-1} M_{x}^{-1}=S_{2} \mathrm{e}^{\pi \mathrm{i} \theta_{\infty} J} S_{1}
$$

(cf. [2, Section 2]), we have $\left(M_{x} M_{0}\right)_{21}=-\mathrm{e}^{-\pi \mathrm{i} \theta_{\infty}} s_{1},\left(M_{x} M_{0}\right)_{12}=-\mathrm{e}^{-\pi \mathrm{i} \theta_{\infty}} s_{2}, \operatorname{tr}\left(M_{x} M_{0}\right)=$ $2 \cos \pi \theta_{\infty}+\mathrm{e}^{-\pi \mathrm{i} \theta \infty} s_{1} s_{2}$. As will be seen in Remark 2.12 and Corollary 2.13, using the relations in the following theorems we may explicitly represent $\left(M_{0}, M_{x}, S_{1}, S_{2}\right)$ for each solution of (1.3) in terms of $\theta_{0}, \theta_{x}, \theta_{\infty}$ and the integration constants $c_{0}, c_{x}, \sigma$. 


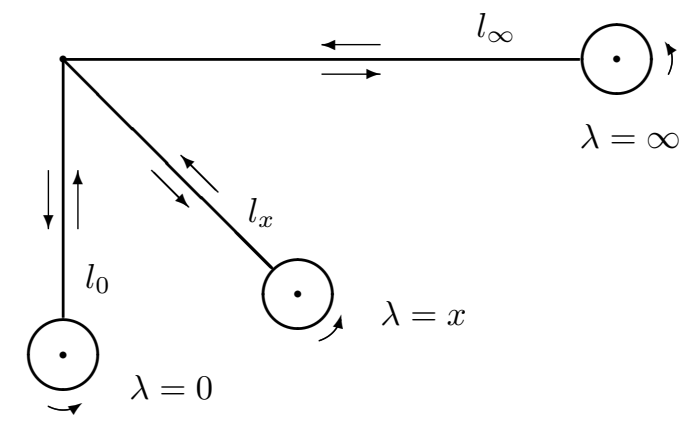

Figure 2.2. $l_{0}, l_{x}$ and $l_{\infty}$.

Theorem 2.10. Suppose that $\theta_{0}, \theta_{x} \notin \mathbb{Z}$. Then, for each $\left(A_{0}(\mathbf{c}, \sigma, x), A_{x}(\mathbf{c}, \sigma, x)\right)$ of Theorem 2.1, the corresponding matrices $M_{0}, M_{x}, S_{1}, S_{2}$ satisfy

$$
\begin{aligned}
& S_{1} M_{x} M_{0} M_{x}^{-1} S_{1}^{-1}=\left(C_{0}^{1}\right)^{-1} \mathrm{e}^{\pi \mathrm{i} \theta_{0} J} C_{0}^{1}, \quad S_{2}^{-1} M_{0} S_{2}=\left(C_{0}^{2}\right)^{-1} \mathrm{e}^{\pi \mathrm{i} \theta_{0} J} C_{0}^{2}, \\
& M_{x}=C_{x}^{-1} \mathrm{e}^{\pi \mathrm{i} \theta_{x} J} C_{x},
\end{aligned}
$$

where $C_{0}^{1}, C_{0}^{2}$ and $C_{x}$ are given by

$$
C_{0}^{1}=V_{0} S_{*}^{-1} \mathrm{e}^{\pi \mathrm{i}\left(\sigma+\theta_{\infty}\right) J / 4} c_{0}^{-J / 2}, \quad C_{0}^{2}=V_{0} S_{* *}^{-1} \mathrm{e}^{-\pi \mathrm{i}\left(\sigma+\theta_{\infty}\right) J / 4} c_{0}^{-J / 2}, \quad C_{x}=V_{x} c_{x}^{-J / 2}
$$

with

$$
\begin{aligned}
V_{0} & =\left(\begin{array}{cc}
\frac{\mathrm{e}^{\pi \mathrm{i}\left(\sigma-2 \theta_{0}-\theta_{\infty}\right) / 4} \Gamma\left(-\theta_{0}\right)}{\Gamma\left(1-\left(\sigma+2 \theta_{0}-\theta_{\infty}\right) / 4\right)} & \frac{\Gamma\left(-\theta_{0}\right)}{\Gamma\left(1+\left(\sigma-2 \theta_{0}-\theta_{\infty}\right) / 4\right)} \\
\frac{\mathrm{e}^{\pi \mathrm{i}\left(\sigma+2 \theta_{0}-\theta_{\infty}\right) / 4} \Gamma\left(\theta_{0}\right)}{\Gamma\left(-\left(\sigma-2 \theta_{0}-\theta_{\infty}\right) / 4\right)} & -\frac{\Gamma\left(\theta_{0}\right)}{\Gamma\left(\left(\sigma+2 \theta_{0}-\theta_{\infty}\right) / 4\right)}
\end{array}\right), \\
V_{x} & =\left.V_{0}\right|_{\left(\sigma, \theta_{0}\right) \mapsto\left(-\sigma, \theta_{x}\right)} \quad \text { i.e., the result of the substitution }\left(\sigma, \theta_{0}\right) \mapsto\left(-\sigma, \theta_{x}\right) \text { in } V_{0}, \\
S_{*} & =I-\frac{2 \pi \mathrm{i}}{\Gamma\left(-\left(\sigma-2 \theta_{0}-\theta_{\infty}\right) / 4\right) \Gamma\left(1-\left(\sigma+2 \theta_{0}-\theta_{\infty}\right) / 4\right)} \Delta_{-}, \\
S_{* *} & =I+\frac{2 \pi \mathrm{ie}}{\Gamma\left(-\pi \mathrm{i}\left(\sigma-\theta_{\infty}\right) / 2\right.} \Delta_{+} .
\end{aligned}
$$

Theorem 2.11. In the case where $\theta_{0}$ or $\theta_{x}$ is an integer, the formulas in Theorem 2.10 are to be replaced as follows:

(1) if $\theta_{0} \in \mathbb{Z}$, then

$$
S_{1} M_{x} M_{0} M_{x}^{-1} S_{1}^{-1}=\left(\hat{C}_{0}^{1}\right)^{-1} \mathrm{e}^{2 \pi \mathrm{i} \Delta_{*}} \hat{C}_{0}^{1}, \quad S_{2}^{-1} M_{0} S_{2}=\left(\hat{C}_{0}^{2}\right)^{-1} \mathrm{e}^{2 \pi \mathrm{i} \Delta_{*}} \hat{C}_{0}^{2},
$$

where $\Delta_{*}$ denotes $\Delta_{+}$if $\theta_{0} \in \mathbb{N} \cup\{0\}$, and $\Delta_{-}$if $-\theta_{0} \in \mathbb{N}$, and $\hat{C}_{0}^{1}$ and $\hat{C}_{0}^{2}$ are given by

$$
\hat{C}_{0}^{1}=\hat{V}_{0} S_{*}^{-1} \mathrm{e}^{\pi \mathrm{i}\left(\sigma+\theta_{\infty}\right) J / 4} c_{0}^{-J / 2}, \quad \hat{C}_{0}^{2}=\hat{V}_{0} S_{* *}^{-1} \mathrm{e}^{-\pi \mathrm{i}\left(\sigma+\theta_{\infty}\right) J / 4} c_{0}^{-J / 2}
$$


with $\hat{V}_{0}$ written in the form

$$
\begin{aligned}
& \hat{V}_{0}=\left(\begin{array}{cc}
\left(\hat{V}_{0}\right)_{11} & \left(\hat{V}_{0}\right)_{12} \\
1 & 1
\end{array}\right) \\
& \times \operatorname{diag}\left[\frac{\mathrm{e}^{\pi \mathrm{i}\left(\sigma+2 \theta_{0}-\theta_{\infty}\right) / 4}}{\theta_{0} ! \Gamma\left(1-\left(\sigma+2 \theta_{0}-\theta_{\infty}\right) / 4\right)} \frac{(-1)^{\theta_{0}}}{\theta_{0} ! \Gamma\left(1+\left(\sigma-2 \theta_{0}-\theta_{\infty}\right) / 4\right)}\right], \\
& \left(\hat{V}_{0}\right)_{11}=\psi\left(-\left(\sigma-2 \theta_{0}-\theta_{\infty}\right) / 4\right)-\psi(1)-\psi\left(1+\theta_{0}\right)-\pi \mathrm{i} \text {, } \\
& \left(\hat{V}_{0}\right)_{12}=\psi\left(1+\left(\sigma+2 \theta_{0}-\theta_{\infty}\right) / 4\right)-\psi(1)-\psi\left(1+\theta_{0}\right)
\end{aligned}
$$

if $\theta_{0} \in \mathbb{N} \cup\{0\}$, and

$$
\begin{aligned}
& \hat{V}_{0}=\left(\begin{array}{cc}
1 & 1 \\
\left(\hat{V}_{0}\right)_{21} & \left(\hat{V}_{0}\right)_{22}
\end{array}\right) \\
& \quad \times \operatorname{diag}\left[\frac{-\mathrm{e}^{\pi \mathrm{i}\left(\sigma-2 \theta_{0}-\theta_{\infty}\right) / 4}}{\left(-\theta_{0}\right) ! \Gamma\left(-\left(\sigma-2 \theta_{0}-\theta_{\infty}\right) / 4\right)} \frac{(-1)^{\theta_{0}}}{\left(-\theta_{0}\right) ! \Gamma\left(\left(\sigma+2 \theta_{0}-\theta_{\infty}\right) / 4\right)}\right], \\
& \left(\hat{V}_{0}\right)_{21}=\psi\left(-\left(\sigma+2 \theta_{0}-\theta_{\infty}\right) / 4\right)-\psi(1)-\psi\left(1-\theta_{0}\right)-\pi \mathrm{i}, \\
& \left(\hat{V}_{0}\right)_{22}=\psi\left(1+\left(\sigma-2 \theta_{0}-\theta_{\infty}\right) / 4\right)-\psi(1)-\psi\left(1-\theta_{0}\right)
\end{aligned}
$$

if $-\theta_{0} \in \mathbb{N}, \psi(t)$ being the di-Gamma function $\psi(t)=\Gamma^{\prime}(t) / \Gamma(t)$;

(2) if $\theta_{x} \in \mathbb{Z}$, then

$$
M_{x}=\hat{C}_{x}^{-1} \mathrm{e}^{2 \pi \mathrm{i} \Delta_{*}} \hat{C}_{x}, \quad \hat{C}_{x}=\hat{V}_{x} c_{x}^{-J / 2},
$$

where $\Delta_{*}$ is as in (1), and $\hat{V}_{x}=\left.\hat{V}_{0}\right|_{\left(\sigma, \theta_{0}\right) \mapsto\left(-\sigma, \theta_{x}\right)}$.

Remark 2.12. Combining $M_{x} M_{0}=S_{1}^{-1} \mathrm{e}^{-\pi \mathrm{i} \theta_{\infty} J} S_{2}^{-1}$ (cf. (2.2)) with the first relation in (2.3) we have

$$
S_{2}^{-1} M_{x}^{-1} S_{1}^{-1}=S_{2}^{-1} C_{x}^{-1} \mathrm{e}^{-\pi \mathrm{i} \theta_{x} J} C_{x} S_{1}^{-1}=\mathrm{e}^{\pi \mathrm{i} \theta_{\infty} J}\left(C_{0}^{1}\right)^{-1} \mathrm{e}^{\pi \mathrm{i} \theta_{0} J} C_{0}^{1},
$$

if $\theta_{0}, \theta_{x} \notin \mathbb{Z}$. The $(2,1)$ - and $(1,2)$-entries of this yield $s_{1}$ and $s_{2}$, respectively, which reveal the Stokes multipliers $S_{1}$ and $S_{2}$, and then $\left(M_{0}, M_{x}, S_{1}, S_{2}\right)$ may be written in terms of $\theta_{0}$, $\theta_{x}, \theta_{\infty}, \sigma, c_{0}, c_{x}$ as in the corollary below (note that $\operatorname{tr} M_{0}=2 \cos \pi \theta_{0}, \operatorname{tr} M_{x}=2 \cos \pi \theta_{x}$, $\left.\operatorname{det} M_{0}=\operatorname{det} M_{x}=1\right)$. In the case where $\theta_{0}$ or $\theta_{x} \in \mathbb{Z}$ as well, these matrices are obtained by the same argument. Such $\left(M_{0}, M_{x}\right)$ is a point on the manifold of the monodromy data (cf. [2, Proposition 2.2, Remark 2.3]).

Corollary 2.13. If $\theta_{0}, \theta_{x} \notin \mathbb{Z}$, then

$$
\begin{aligned}
\left(M_{0}\right)_{11}= & \mathrm{e}^{\pi \mathrm{i}\left(\sigma-\theta_{\infty}\right) / 2}\left(1-\frac{2 \pi \mathrm{i} c_{0}^{-1}}{\Gamma\left(1-\left(\sigma+2 \theta_{0}-\theta_{\infty}\right) / 4\right) \Gamma\left(-\left(\sigma-2 \theta_{0}-\theta_{\infty}\right) / 4\right)}\right. \\
& \left.\times \frac{2 \pi \mathrm{i} c_{x}}{\Gamma\left(1-\left(\sigma+2 \theta_{x}+\theta_{\infty}\right) / 4\right) \Gamma\left(-\left(\sigma-2 \theta_{x}+\theta_{\infty}\right) / 4\right)}\right), \\
\left(M_{0}\right)_{21}= & \frac{2 \pi \mathrm{ie}}{\Gamma\left(1-\left(\sigma+2 \theta_{\infty} c_{0}^{-1}\right.\right.}, \\
\left(M_{x}\right)_{11}=\mathrm{e}^{-\pi \mathrm{i}\left(\sigma+\theta_{\infty}\right) / 2}, & \\
\left(M_{x}\right)_{12}= & \frac{2 \pi \mathrm{i} c_{x}}{\Gamma\left(1-\left(\sigma+2 \theta_{x}+\theta_{\infty}\right) / 4\right) \Gamma\left(-\left(\sigma-2 \theta_{x}+\theta_{\infty}\right) / 4\right)},
\end{aligned}
$$




$$
\begin{aligned}
s_{1}= & -\frac{2 \pi \mathrm{ie}^{\pi \mathrm{i}\left(\sigma+\theta_{\infty}\right) / 2} c_{0}^{-1}}{\Gamma\left(1-\left(\sigma+2 \theta_{0}-\theta_{\infty}\right) / 4\right) \Gamma\left(-\left(\sigma-2 \theta_{0}-\theta_{\infty}\right) / 4\right)} \\
& -\frac{2 \pi \mathrm{i} c_{x}^{-1}}{\Gamma\left(1+\left(\sigma-2 \theta_{x}+\theta_{\infty}\right) / 4\right) \Gamma\left(\left(\sigma+2 \theta_{x}+\theta_{\infty}\right) / 4\right)}, \\
s_{2}= & -\frac{2 \pi \mathrm{ie}^{\pi \mathrm{i} \theta_{\infty}} c_{0}}{\Gamma\left(1+\left(\sigma-2 \theta_{0}-\theta_{\infty}\right) / 4\right) \Gamma\left(\left(\sigma+2 \theta_{0}-\theta_{\infty}\right) / 4\right)} \\
& -\frac{2 \pi \mathrm{ie}^{\pi \mathrm{i}\left(\sigma+\theta_{\infty}\right) / 2} c_{x}}{\Gamma\left(1-\left(\sigma+2 \theta_{x}+\theta_{\infty}\right) / 4\right) \Gamma\left(-\left(\sigma-2 \theta_{x}+\theta_{\infty}\right) / 4\right)} .
\end{aligned}
$$

Remark 2.14. The results above combined with the monodromy data for solutions of (V) around $x=0[2,14,24]$ yield the parametric connection formula between $x=0$ and $x=\mathrm{i} \infty$ (cf. Remark 2.19), which corresponds to that for $\tau_{\mathrm{V}}(x)$ of [19, Conjecture $\left.\mathrm{C}\right]$.

Suppose that, for every $k \in \mathbb{Z} \backslash\{0\},\left(A_{0}^{(k)}\left(\mathbf{c}^{(k)}, \sigma^{(k)}, x\right), A_{x}^{(k)}\left(\mathbf{c}^{(k)}, \sigma^{(k)}, x\right)\right)$ with $\left(\mathbf{c}^{(k)}, \sigma^{(k)}\right)=$ $\left(c_{0}^{(k)}, c_{x}^{(k)}, \sigma^{(k)}\right) \in(\mathbb{C} \backslash\{0\})^{2} \times \mathbb{C}$ is the analytic continuation of $\left(A_{0}(\mathbf{c}, \sigma, x), A_{x}(\mathbf{c}, \sigma, x)\right)$ to the domain $\Sigma_{k}\left(\sigma^{(k)}, \varepsilon, x_{\infty}, \delta\right.$ ) (cf. Remark 2.6). For every $j \in \mathbb{Z}$, let $l_{0}^{(j)}$ and $l_{x}^{(j)}$ be the loops in the $\lambda$-plane defined for $(2 j-1) \pi<\arg x<(2 j+1) \pi$ in the same way as in Fig. 2.2, and let $\left(M_{0}^{(k)}, M_{x}^{(k)}\right)$ with $k=2 j$ or $2 j-1$ correspond to $\left(A_{0}^{(k)}\left(\mathbf{c}^{(k)}, \sigma^{(k)}, x\right), A_{x}^{(k)}\left(\mathbf{c}^{(k)}, \sigma^{(k)}, x\right)\right)$ for $x \in \Sigma_{k}\left(\sigma^{(k)}, \varepsilon, x_{\infty}, \delta\right)$, where $M_{0}^{(k)}$ and $M_{x}^{(k)}$ are the monodromy matrices given by the analytic continuation of $Y(x, \lambda)$ along $l_{0}^{(j)}$ and $l_{x}^{(j)}$, respectively (note that $l_{0}^{(0)}=l_{0}, l_{x}^{(0)}=l_{x}, M_{0}^{(0)}=M_{0}$, $\left.M_{x}^{(0)}=M_{x}\right)$. Then by definition, for every $j \in \mathbb{Z}$,

$$
\begin{gathered}
\left(M_{0}^{(2 j)}, M_{x}^{(2 j)}\right)=\left(M_{0}^{(2 j)}, M_{x}^{(2 j)}\right)\left(\mathbf{c}^{(2 j)}, \sigma^{(2 j)}\right)=\left.\left(M_{0}, M_{x}\right)\right|_{(\mathbf{c}, \sigma) \mapsto\left(\mathbf{c}^{(2 j)}, \sigma^{(2 j)}\right)}, \\
\begin{aligned}
\left(M_{0}^{(2 j-1)}, M_{x}^{(2 j-1)}\right) & =\left(M_{0}^{(2 j-1)}, M_{x}^{(2 j-1)}\right)\left(\mathbf{c}^{(2 j-1)}, \sigma^{(2 j-1)}\right) \\
& =\left.\left(M_{0}^{(-1)}, M_{x}^{(-1)}\right)\right|_{\left(\mathbf{c}^{(-1)}, \sigma^{(-1)}\right) \mapsto\left(\mathbf{c}^{(2 j-1)}, \sigma^{(2 j-1)}\right)} .
\end{aligned}
\end{gathered}
$$

Remark 2.15. For $\left(A_{0}^{(-1)}\left(\mathbf{c}^{(-1)}, \sigma^{(-1)}, x\right), A_{x}^{(-1)}\left(\mathbf{c}^{(-1)}, \sigma^{(-1)}, x\right)\right)$ in $\Sigma_{-1}\left(\sigma^{(-1)}, \varepsilon, x_{\infty}, \delta\right)$ the corresponding matrices $M_{0}^{(-1)}$ and $M_{x}^{(-1)}$ are defined along the loops $l_{0}$ and $l_{x}$, respectively, as in Fig. 2.2 for $-\pi<\arg x<\pi$. If $\theta_{0}, \theta_{x} \notin \mathbb{Z}$,

$$
\begin{aligned}
& M_{0}^{(-1)}=C_{0}^{-1} \mathrm{e}^{\pi \mathrm{i} \theta_{0} J} C_{0}, \\
& S_{1} M_{x}^{(-1)} S_{1}^{-1}=\left(C_{x}^{1}\right)^{-1} \mathrm{e}^{\pi \mathrm{i} \theta_{x} J} C_{x}^{1}, \quad S_{2}\left(M_{0}^{(-1)}\right)^{-1} M_{x}^{(-1)} M_{0}^{(-1)} S_{2}^{-1}=\left(C_{x}^{2}\right)^{-1} \mathrm{e}^{\pi \mathrm{i} \theta_{x} J} C_{x}^{2},
\end{aligned}
$$

where

$$
\begin{aligned}
& C_{0}=\tilde{V}_{0} \mathrm{e}^{-\pi \mathrm{i}\left(\sigma+\theta_{\infty}\right) J / 4}\left(c_{0}^{(-1)}\right)^{-J / 2}, \quad C_{x}^{1}=\tilde{V}_{x} \tilde{S}_{*}^{-1}\left(c_{x}^{(-1)}\right)^{-J / 2}, \\
& C_{x}^{2}=\tilde{V}_{x} \tilde{S}_{* *}^{-1} \mathrm{e}^{\pi \mathrm{i}\left(\sigma-\theta_{\infty}\right) J / 2}\left(c_{x}^{(-1)}\right)^{-J / 2}
\end{aligned}
$$

with

$$
\begin{aligned}
& \tilde{V}_{0}=\left.V_{0}\right|_{\sigma \mapsto \sigma^{(-1)}}, \quad \tilde{V}_{x}=\left.V_{x}\right|_{\sigma \mapsto \sigma^{(-1)}}, \\
& \tilde{S}_{*}=\left.S_{*}\right|_{\left(\sigma, \theta_{0}\right) \mapsto\left(-\sigma^{(-1)}, \theta_{x}\right)}, \quad \tilde{S}_{* *}=\left.S_{* *}\right|_{\left(\sigma, \theta_{0}\right) \mapsto\left(-\sigma^{(-1)}, \theta_{x}\right)} .
\end{aligned}
$$

If $\theta_{0}$ or $\theta_{x} \in \mathbb{Z}$, then $\tilde{V}_{0}$ or $\tilde{V}_{x}$ is to be replaced by $\left.\hat{V}_{0}\right|_{\sigma \mapsto \sigma^{(-1)}}$ or $\left.\hat{V}_{x}\right|_{\sigma \mapsto \sigma^{(-1)}}$, respectively, as in Theorem 2.11 (cf. Section 7.5). The isomonodromy property implies $\left(M_{0}, M_{x}\right)(\mathbf{c}, \sigma)=$ $\left(M_{0}^{(-1)}, M_{x}^{(-1)}\right)\left(\mathbf{c}^{(-1)}, \sigma^{(-1)}\right)$, which gives the relation between $\left(\mathbf{c}^{(-1)}, \sigma^{(-1)}\right)$ and $(\mathbf{c}, \sigma)$. 
The following proposition gives the connection formulas between $\left(\mathbf{c}^{(k \pm 2)}, \sigma^{(k \pm 2)}\right)$ and $\left(\mathbf{c}^{(k)}, \sigma^{(k)}\right)$. This is obtained by deformation of the loops $l_{0}^{(j)}, l_{x}^{(j)}$ or by action of the braid $\beta_{1}^{2}$ (see [4, Section 1.2.3], [11, p. 331]).

Proposition 2.16. For every $k \in \mathbb{Z}$

$$
\begin{aligned}
& M_{0}^{(k+2)}=M_{x}^{(k)} M_{0}^{(k)}\left(M_{x}^{(k)}\right)^{-1}, \quad M_{x}^{(k+2)}=M_{x}^{(k)} M_{0}^{(k)} M_{x}^{(k)}\left(M_{0}^{(k)}\right)^{-1}\left(M_{x}^{(k)}\right)^{-1}, \\
& M_{0}^{(k-2)}=\left(M_{0}^{(k)}\right)^{-1}\left(M_{x}^{(k)}\right)^{-1} M_{0}^{(k)} M_{x}^{(k)} M_{0}^{(k)}, \quad M_{x}^{(k-2)}=\left(M_{0}^{(k)}\right)^{-1} M_{x}^{(k)} M_{0}^{(k)} .
\end{aligned}
$$

Remark 2.17. For the solution $\left(A_{0}(\mathbf{c}, x), A_{x}(\mathbf{c}, x)\right)$ with $\sigma_{0}=-2 \theta_{x}-\theta_{\infty}$ in Theorem 2.8, the corresponding monodromy matrices are given by $\left.\left(M_{0}, M_{x}\right)\right|_{\sigma=\sigma_{0}=-2 \theta_{x}-\theta_{\infty}}$. For the solution $\left(A_{0}\left(c_{0}, x\right), A_{x}\left(c_{0}, x\right)\right)$ we have $\left.\left(M_{0}, \mathrm{e}^{\pi \mathrm{i} \theta_{x} J}\right)\right|_{\left(c_{x}, \sigma\right)=\left(0, \sigma_{0}\right)}$.

\subsection{Fifth Painlevé transcendents, zeros and poles}

From Theorem 2.1 and (1.2) we may derive a solution of $(\mathrm{V})$ written in the form

$$
y=\frac{g_{+}(\mathbf{c}, \sigma, x)\left(f_{0}(\mathbf{c}, \sigma, x)+\theta_{0} / 2\right)}{f_{+}(\mathbf{c}, \sigma, x)\left(g_{0}(\mathbf{c}, \sigma, x)+\theta_{x} / 2\right)}
$$

parametrised by $(c, \sigma)=\left(c_{x} / c_{0}, \sigma\right)$ or $\left(c^{\prime}, \sigma\right)=\left(c_{0} / c_{x}, \sigma\right)$. This is meromorphic in $\Sigma\left(\sigma, \varepsilon, x_{\infty}, \delta\right)$ and is expanded into a convergent series in a subdomain of $\Sigma\left(\sigma, \varepsilon, x_{\infty}, \delta\right)$. Let $\delta$ be a given positive number such that $\delta<\pi / 2$.

Theorem 2.18. Let $B \subset \mathbb{C} \backslash\{0\}$ and $B_{*} \subset \mathbb{C}$ be given domains. Suppose that $\operatorname{dist}\left(\left\{-2 \theta_{0}+\right.\right.$ $\left.\left.\theta_{\infty}, 2 \theta_{x}-\theta_{\infty}\right\}, B_{*}\right)>0$. Then $(\mathrm{V})$ admits a two-parameter family of solutions $\{y(c, \sigma, x) ;(c, \sigma) \in$ $\left.B \times B_{*}\right\}$ such that $y(c, \sigma, x)$ is holomorphic in $(c, \sigma, x) \in B \times D\left(B_{*}, \varepsilon^{\prime}, x_{\infty}^{\prime}, \delta\right)$ and expanded into the convergent series in $\left(\mathrm{e}^{x} x^{\sigma-1}, \mathrm{e}^{-x} x^{-\sigma-1}\right)$

$$
\begin{aligned}
y(c, \sigma, x)= & c\left(1+\left[x^{-1}\right]_{*}\right) \mathrm{e}^{x} x^{\sigma} \\
& \times\left(1+\sum_{n=1}^{\infty}\left(a_{n}+\left[x^{-1}\right]_{*}\right)\left(\mathrm{e}^{x} x^{\sigma-1}\right)^{n}+\sum_{n=1}^{\infty}\left(b_{n}+\left[x^{-1}\right]_{*}\right)\left(\mathrm{e}^{-x} x^{-\sigma-1}\right)^{n}\right)
\end{aligned}
$$

with $a_{n}, b_{n} \in \mathbb{Q}_{0}:=\mathbb{Q}\left[\theta_{0}, \theta_{x}, \theta_{\infty}, c, c^{-1}, \sigma,\left(\sigma+2 \theta_{0}-\theta_{\infty}\right)^{-1},\left(-\sigma+2 \theta_{x}-\theta_{\infty}\right)^{-1}\right]$, in particular,

$$
a_{1}=c\left(-\sigma+\theta_{0}+\theta_{x}\right) / 2, \quad b_{1}=c^{-1}\left(\sigma+\theta_{0}+\theta_{x}\right) / 2 .
$$

Here $\varepsilon^{\prime}=\varepsilon^{\prime}\left(B, B_{*}, \delta\right)$ (respectively, $\left.x_{\infty}^{\prime}=x_{\infty}^{\prime}\left(B, B_{*}, \delta\right)\right)$ is a sufficiently small (respectively, large) positive number depending on $\left(B, B_{*}, \delta\right)$, and each $\left[x^{-1}\right]_{*}$ is represented by an asymptotic series with coefficients in $\mathbb{Q}_{0}$ valid in $|\arg x-\pi / 2|<\pi / 2-\delta,|x|>x_{\infty}^{\prime}$.

Remark 2.19. For $y(c, \sigma, x)$ the corresponding monodromy matrices are obtained by putting $\left(c_{0}, c_{x}\right)=(1, c)$ in $M_{0}, M_{x}$ of Theorem 2.10.

Remark 2.20. The solution $y(c, \sigma, x)$ corresponds to $y_{\mathrm{V}, 0, *}(\mathbf{c}, x)$ of $[22$, Theorem 2.10 and Section 2.3] (see also [26]) and converges in the domain larger than the previously known one (note that $\left.(\mathrm{V})\right|_{x \mapsto \mathrm{i} x}$ is treated in [22]).

Theorem 2.21. Let $\tilde{B} \subset \mathbb{C}$ be a given domain. 
(1) Suppose that $\theta_{x}\left(\theta_{0}-\theta_{x}-\theta_{\infty}\right) \neq 0$, and set $\sigma_{0}=-2 \theta_{x}-\theta_{\infty}$. Then (V) admits a oneparameter family of solutions $\left\{y_{+}(c, x) ; c \in \tilde{B}\right\}$ such that $y_{+}(c, x)$ is holomorphic in $(c, x) \in$ $\tilde{B} \times \Sigma_{*}\left(\varepsilon^{\prime}, x_{\infty}^{\prime}, \delta\right)$ and is expanded into a convergent series in $\mathrm{e}^{x} x^{\sigma_{0}-1}$ of the form

$$
\begin{aligned}
y_{+}(c, x)= & \frac{1}{2}\left(\theta_{0}-\theta_{x}-\theta_{\infty}\right) x^{-1}\left(1+\left[x^{-1}\right]_{*}\right) \\
& +c\left(1+\left[x^{-1}\right]_{*}\right) \mathrm{e}^{x} x^{\sigma_{0}}\left(1+\sum_{n=1}^{\infty}\left(\tilde{a}_{n}+\left[x^{-1}\right]_{*}\right)\left(\mathrm{e}^{x} x^{\sigma_{0}-1}\right)^{n}\right)
\end{aligned}
$$

with $\tilde{a}_{n} \in \mathbb{Q}_{1}:=\mathbb{Q}\left[\theta_{0}, \theta_{x}, \theta_{\infty}, c,\left(\theta_{0}-\theta_{x}-\theta_{\infty}\right)^{-1}, \theta_{x}^{-1}\right]$. Here $\varepsilon^{\prime}=\varepsilon^{\prime}(\tilde{B}, \delta)$ (respectively, $\left.x_{\infty}^{\prime}=x_{\infty}^{\prime}(\tilde{B}, \delta)\right)$ is a sufficiently small (respectively, large) positive number depending on $(\tilde{B}, \delta)$, and each $\left[x^{-1}\right]_{*}$ is represented by an asymptotic series with coefficients in $\mathbb{Q}_{1}$ valid in $-(\pi / 2-\delta)<\arg x-\pi / 2<\pi-\delta,|x|>x_{\infty}^{\prime}$.

(2) Suppose that $\theta_{0}\left(\theta_{0}-\theta_{x}+\theta_{\infty}\right) \neq 0$, and set $\sigma_{0}^{\prime}=2 \theta_{0}+\theta_{\infty}$. Then $(\mathrm{V})$ admits a oneparameter family of solutions $\left\{y_{-}\left(c^{\prime}, x\right) ; c^{\prime} \in \tilde{B}\right\}$ such that $y_{-}\left(c^{\prime}, x\right)$ is holomorphic in $\left(c^{\prime}, x\right) \in \tilde{B} \times \Sigma_{*}^{\prime}\left(\varepsilon^{\prime \prime}, x_{\infty}^{\prime \prime}, \delta\right)$ and that the reciprocal is expanded into a convergent series in $\mathrm{e}^{-x} x^{-\sigma_{0}^{\prime}-1}$ of the form

$$
\begin{aligned}
1 / y_{-}\left(c^{\prime}, x\right)= & \frac{1}{2}\left(\theta_{0}-\theta_{x}+\theta_{\infty}\right) x^{-1}\left(1+\left[x^{-1}\right]_{*}\right) \\
& +c^{\prime}\left(1+\left[x^{-1}\right]_{*}\right) \mathrm{e}^{-x} x^{-\sigma_{0}^{\prime}}\left(1+\sum_{n=1}^{\infty}\left(\tilde{b}_{n}+\left[x^{-1}\right]_{*}\right)\left(\mathrm{e}^{-x} x^{-\sigma_{0}^{\prime}-1}\right)^{n}\right)
\end{aligned}
$$

with $\tilde{b}_{n} \in \mathbb{Q}_{2}:=\mathbb{Q}\left[\theta_{0}, \theta_{x}, \theta_{\infty}, c^{\prime},\left(\theta_{0}-\theta_{x}+\theta_{\infty}\right)^{-1}, \theta_{0}^{-1}\right]$. Here $\varepsilon^{\prime \prime}=\varepsilon^{\prime \prime}(\tilde{B}, \delta)$ (respectively, $\left.x_{\infty}^{\prime \prime}=x_{\infty}^{\prime \prime}(\tilde{B}, \delta)\right)$ is a sufficiently small (respectively, large) positive number depending on $(\tilde{B}, \delta)$, and each $\left[x^{-1}\right]_{*}$ is represented by an asymptotic series with coefficients in $\mathbb{Q}_{2}$ valid in $-(\pi-\delta)<\arg x-\pi / 2<\pi / 2-\delta,|x|>x_{\infty}^{\prime \prime}$.

Remark 2.22. The reciprocal $1 / y_{-}\left(c^{\prime}, x\right)$ itself solves $(\mathrm{V})$ with $\left(\theta_{0}, \theta_{x},-\theta_{\infty}\right)$.

Remark 2.23. There exist the asymptotic solutions $y_{+}(0, x)=(1 / 2)\left(\theta_{0}-\theta_{x}-\theta_{\infty}\right) x^{-1}(1+$ $\left.\left[x^{-1}\right]_{*}\right)$ and $y_{-}(0, x)=2\left(\theta_{0}-\theta_{x}+\theta_{\infty}\right)^{-1} x\left(1+\left[x^{-1}\right]_{*}\right)$ in the sector $|\arg x-\pi / 2|<\pi-\delta$.

Remark 2.24. For $\sigma=2 \theta_{x}-\theta_{\infty}$ (respectively, $\sigma=-2 \theta_{0}+\theta_{\infty}$ ) as well, under the condition $\left(\theta_{0}^{2}-\left(\theta_{x}-\theta_{\infty}\right)^{2}\right) \theta_{x} \neq 0$ (respectively, $\left(\theta_{x}^{2}-\left(\theta_{0}-\theta_{\infty}\right)^{2}\right) \theta_{0} \neq 0$ ), there exists a family of solutions $\left\{\tilde{y}_{-}\left(c^{\prime}, x\right) ; c^{\prime} \in \tilde{B}\right\}$ (respectively, $\left.\left\{\tilde{y}_{+}(c, x) ; c \in \tilde{B}\right\}\right)$ such that

$$
\begin{aligned}
1 / \tilde{y}_{-}\left(c^{\prime}, x\right)= & -\frac{1}{2}\left(\theta_{0}-\theta_{x}+\theta_{\infty}\right) x^{-1}\left(1+\left[x^{-1}\right]_{*}\right) \\
& +c^{\prime}\left(1+\left[x^{-1}\right]_{*}\right) \mathrm{e}^{-x} x^{-2 \theta_{x}+\theta_{\infty}}\left(1+\sum_{n=1}^{\infty}\left(\tilde{\tilde{b}}_{n}+\left[x^{-1}\right]_{*}\right)\left(\mathrm{e}^{-x} x^{-2 \theta_{x}+\theta_{\infty}-1}\right)^{n}\right)
\end{aligned}
$$

$\left(\right.$ respectively, $\quad \tilde{y}_{+}(c, x)=-\frac{1}{2}\left(\theta_{0}-\theta_{x}-\theta_{\infty}\right) x^{-1}\left(1+\left[x^{-1}\right]_{*}\right)$

$$
\left.+c\left(1+\left[x^{-1}\right]_{*}\right) \mathrm{e}^{x} x^{-2 \theta_{0}+\theta_{\infty}}\left(1+\sum_{n=1}^{\infty}\left(\tilde{\tilde{a}}_{n}+\left[x^{-1}\right]_{*}\right)\left(\mathrm{e}^{x} x^{-2 \theta_{0}+\theta_{\infty}-1}\right)^{n}\right)\right)
$$

in $\Sigma_{*}^{\prime}\left(\varepsilon^{\prime \prime}, x_{\infty}^{\prime \prime}, \delta\right)$ (respectively, $\left.\Sigma_{*}\left(\varepsilon^{\prime}, x_{\infty}^{\prime}, \delta\right)\right)$.

Remark 2.25. Using (1.2) we may derive convergent series representations for $z(x)$ and $u(x)=$ $x^{-\theta_{\infty}} u_{\mathrm{AK}}(x)$, which are parametrised by $\left(\sigma, c_{x} / c_{0}\right)$ and $\left(\sigma, c_{x} / c_{0}, c_{0}\right)$, respectively. 
From the quotient expression (2.6) we obtain a sequence of zeros or poles of $y(c, \sigma, x)$.

Theorem 2.26. Let $R_{0}$ be a given positive number.

(1) Suppose that $\theta_{x}\left(\theta_{0} \pm \theta_{x}-\theta_{\infty}\right) \neq 0$. Then there exists a small positive number $\varepsilon_{0}=$ $\varepsilon_{0}\left(R_{0}\right)=\varepsilon_{0}\left(R_{0}, \theta_{0}, \theta_{x}, \theta_{\infty}\right)$ such that, for every $(c, \sigma) \in \mathbb{C}^{2}$ satisfying $0<|c|<R_{0}$, $\sigma \neq \pm 2 \theta_{0}+\theta_{\infty}, \pm 2 \theta_{x}-\theta_{\infty},\left|\sigma+2 \theta_{x}+\theta_{\infty}\right|<R_{0}|c|$ (respectively, $\left.\left|\sigma+2 \theta_{0}-\theta_{\infty}\right|<R_{0}|c|\right)$ and $\left|4 c /\left(\sigma+2 \theta_{0}-\theta_{\infty}\right)\right|<\varepsilon_{0}$ (respectively, $\left.\left|4 c /\left(\sigma+2 \theta_{x}+\theta_{\infty}\right)\right|<\varepsilon_{0}\right)$, the solution $y(c, \sigma, x)$ has a sequence of zeros $\left\{x_{m}^{(0)}\right\}, m \geq m_{0}$, such that

$$
x_{m}^{(0)}=2 m \pi \mathrm{i}-(\sigma+1) \log (2 m \pi \mathrm{i})-\log \left(\rho_{0}(\sigma) c\right)+O\left(m^{-1} \log m\right),
$$

where $m_{0}$ is some large positive integer and $\rho_{0}(\sigma)=-4 /\left(\sigma+2 \theta_{0}-\theta_{\infty}\right)$ (respectively, $\left.-4 /\left(\sigma+2 \theta_{x}+\theta_{\infty}\right)\right)$.

(2) Suppose that $\theta_{0}\left( \pm \theta_{0}-\theta_{x}+\theta_{\infty}\right) \neq 0$. Then there exists a small positive number $\varepsilon_{0}^{\prime}=$ $\varepsilon_{0}^{\prime}\left(R_{0}\right)=\varepsilon_{0}^{\prime}\left(R_{0}, \theta_{0}, \theta_{x}, \theta_{\infty}\right)$ such that, for every $(c, \sigma) \in \mathbb{C}^{2}$ satisfying $|c|>1 / R_{0}, \sigma \neq$ $\pm 2 \theta_{0}+\theta_{\infty}, \pm 2 \theta_{x}-\theta_{\infty},\left|c\left(\sigma-2 \theta_{0}-\theta_{\infty}\right)\right|<R_{0}$ (respectively, $\left.\left|c\left(\sigma-2 \theta_{x}+\theta_{\infty}\right)\right|<R_{0}\right)$ and $\left|4 c^{-1} /\left(\sigma-2 \theta_{x}+\theta_{\infty}\right)\right|<\varepsilon_{0}^{\prime}$ (respectively, $\left.\left|4 c^{-1} /\left(\sigma-2 \theta_{0}-\theta_{\infty}\right)\right|<\varepsilon_{0}^{\prime}\right)$, the solution $y(c, \sigma, x)$ has a sequence of poles $\left\{x_{m}^{(\infty)}\right\}, m \geq m_{0}$, such that

$$
x_{m}^{(\infty)}=2 m \pi \mathrm{i}-(\sigma-1) \log (2 m \pi \mathrm{i})-\log \left(\rho_{\infty}(\sigma) c\right)+O\left(m^{-1} \log m\right),
$$

where $\rho_{\infty}(\sigma)=-\left(\sigma-2 \theta_{x}+\theta_{\infty}\right) / 4$ (respectively, $\left.-\left(\sigma-2 \theta_{0}-\theta_{\infty}\right) / 4\right)$.

For one-parameter solutions we have

Theorem 2.27. Let $y_{+}(c, x)$ and $y_{-}\left(c^{\prime}, x\right)$ be the solutions given above.

(1) Suppose that $\theta_{x}\left(\theta_{0}-\theta_{x}-\theta_{\infty}\right) \neq 0$. If $c \neq 0$ is sufficiently small, then $y_{+}(c, x)$ has a sequence of zeros $\left\{x_{m}^{+(0)}\right\}, m \geq m_{0}$, such that

$$
x_{m}^{+(0)}=2 m \pi \mathrm{i}-(\sigma+1) \log (2 m \pi \mathrm{i})-\log \left(-2 c /\left(\theta_{0}-\theta_{x}-\theta_{\infty}\right)\right)+O\left(m^{-1} \log m\right),
$$

where $m_{0}$ is some large positive integer.

(2) Suppose that $\theta_{0}\left(\theta_{x}-\theta_{0}-\theta_{\infty}\right) \neq 0$. If $c^{\prime} \neq 0$ is sufficiently small, then $y_{-}\left(c^{\prime}, x\right)$ has a sequence of poles $\left\{x_{m}^{-(\infty)}\right\}, m \geq m_{0}$, such that

$$
x_{m}^{-(\infty)}=2 m \pi \mathrm{i}-(\sigma-1) \log (2 m \pi \mathrm{i})+\log \left(2 c^{\prime} /\left(\theta_{x}-\theta_{0}-\theta_{\infty}\right)\right)+O\left(m^{-1} \log m\right) .
$$

Remark 2.28. To $y(c, \sigma, x)$ of Theorem 2.18 applying the Bäcklund transformation and the substitution $\pi$ : $\left(\theta_{0}-\theta_{x}, \theta_{0}+\theta_{x}, \theta_{\infty}\right) \mapsto\left(1-\theta_{\infty}, 1-\theta_{0}+\theta_{x}, \theta_{0}+\theta_{x}-1\right)$, we obtain another solution of $(\mathrm{V})$ given by

$$
\hat{y}(c, \sigma, x)^{\pi}=\frac{Y(x, y(c, \sigma, x))^{\pi}}{1+Y(x, y(c, \sigma, x))^{\pi}}
$$

with

$$
\begin{aligned}
Y(x, y) & =x^{-1}(y-1)\left(\left(A_{x}\right)_{11}+\theta_{x} / 2-\left(\left(A_{x}\right)_{11}-\theta_{x} / 2\right) y^{-1}\right) \\
& =-2\left(A_{x}\right)_{11} x^{-1}+\left(\left(A_{x}\right)_{11}+\theta_{x} / 2\right) x^{-1} y+\left(\left(A_{x}\right)_{11}-\theta_{x} / 2\right) x^{-1} y^{-1}
\end{aligned}
$$


(cf. [10], [24, Lemma 6.1]). This is expressed as

$$
\begin{aligned}
\hat{y}(c, \sigma, x)^{\pi}= & {\left[x^{-1}\right]-\left(\left(\sigma+2 \theta_{0}-\theta_{\infty}-1\right) / 4+\left[x^{-1}\right]\right) c \mathrm{e}^{x} x^{\sigma-1}+\sum_{n=2}^{\infty}[1]_{*}\left(\mathrm{e}^{x} x^{\sigma-1}\right)^{n} } \\
& -\left(\left(\sigma+2 \theta_{x}+\theta_{\infty}-1\right) / 4+\left[x^{-1}\right]\right) c^{-1} \mathrm{e}^{-x} x^{-\sigma-1}+\sum_{n=2}^{\infty}[1]_{*}\left(\mathrm{e}^{-x} x^{-\sigma-1}\right)^{n}
\end{aligned}
$$

for $\left|\mathrm{e}^{x} x^{\sigma-1}\right|<\varepsilon^{\prime \prime},\left|\mathrm{e}^{-x} x^{-\sigma-1}\right|<\varepsilon^{\prime \prime}, \varepsilon^{\prime \prime}$ being sufficiently small, and admits a sequence of zeros $\left\{\tilde{x}_{m}\right\}$ with $\tilde{x}_{m}=-\log c+2 m \pi \mathrm{i}-\sigma \log (2 m \pi \mathrm{i})+O\left(m^{-1} \log m\right)$ in the domain $\left|\mathrm{e}^{x} x^{\sigma}\right|,\left|\mathrm{e}^{-x} x^{-\sigma}\right| \ll 1$.

\section{$3 \quad$ Families of series}

\section{$3.1 \quad$ Family $\mathfrak{A}$}

Let $B_{0}, B_{x}$ and $B_{*}$ be as in Section 2 , and $\Sigma_{0}\left(x_{\infty}, \delta\right)$ the sector $|\arg x-\pi / 2|<\pi / 2-\delta,|x|>x_{\infty}$. Denote by $\hat{\mathfrak{A}}=\hat{\mathfrak{A}}\left(B_{0}, B_{x}, B_{*}, \Sigma_{0}\left(x_{\infty}, \delta\right)\right)$ the family of pairs $\left(\phi,\left\{p_{n}^{+}(x), p_{n}^{-}(x), p_{0}(x)\right\}_{n \in \mathbb{N}}\right)$, where $\phi$ is a formal series of the form

$$
\phi=\phi(\mathbf{c}, \sigma, x)=\sum_{n=1}^{\infty} p_{n}^{+}(x)\left(\mathrm{e}^{x} x^{\sigma-1}\right)^{n}+\sum_{n=1}^{\infty} p_{n}^{-}(x)\left(\mathrm{e}^{-x} x^{-\sigma-1}\right)^{n}+p_{0}(x) x^{-1},
$$

and $p_{n}^{+}(x), p_{n}^{-}(x)$ and $p_{0}(x)$ are holomorphic in $(\mathbf{c}, \sigma, x)=\left(c_{0}, c_{x}, \sigma, x\right) \in B_{0} \times B_{x} \times B_{*} \times \Sigma_{0}\left(x_{\infty}, \delta\right)$ and admit asymptotic representations

$$
p_{n}^{+}(x) \sim \sum_{m=0}^{\infty} p_{n m}^{+} x^{-m}, \quad p_{n}^{-}(x) \sim \sum_{m=0}^{\infty} p_{n m}^{-} x^{-m}, \quad p_{0}(x) \sim \sum_{m=0}^{\infty} p_{0 m} x^{-m}
$$

with coefficients $p_{n m}^{ \pm}=p_{n m}^{ \pm}(\mathbf{c}, \sigma), p_{0 m}=p_{0 m}(\mathbf{c}, \sigma) \in \mathbb{Q}_{*}$ uniformly in $(\mathbf{c}, \sigma) \in B_{0} \times B_{x} \times B_{*}$ as $x \rightarrow \infty$ through the sector $\Sigma_{0}\left(x_{\infty}, \delta\right)$. Note the example $p_{1}(x) \mathrm{e}^{x} x^{\sigma-1}+p_{0}(x) x^{-1} \equiv 0 \cdot \mathrm{e}^{x} x^{\sigma-1}+$ $0 \cdot x^{-1}$ with $p_{1}(x)=\mathrm{e}^{2 \mathrm{i} x / \delta} \sim 0, p_{0}(x)=-\mathrm{e}^{(2 \mathrm{i} / \delta+1) x} x^{\sigma} \sim 0$ in $\Sigma_{0}\left(x_{\infty}, \delta\right)$. To avoid such an ambiguity $\hat{\mathfrak{A}}$ is defined as the set of the pairs as above. For simplicity, however, keeping the strict definition above in mind, we regard and deal with $\hat{\mathfrak{A}}$ as the family of the formal series $\phi=\phi(\mathbf{c}, \sigma, x)$. To $\phi \in \hat{\mathfrak{A}}$ written as above, we assign the function

$$
\begin{aligned}
\|\phi\| & =\|\phi\|(x, \eta)=\|\phi\|_{\mathbf{c}, \sigma}(x, \eta) \\
& =\sum_{n=1}^{\infty} M\left(p_{n}^{+},|x|\right)\left|\eta x^{-1}\right|^{n}+\sum_{n=1}^{\infty} M\left(p_{n}^{-},|x|\right)\left|\eta^{-1} x^{-1}\right|^{n}+M\left(p_{0},|x|\right)|x|^{-1},
\end{aligned}
$$

where $M(p,|x|)$ is a function of $(\mathbf{c}, \sigma,|x|)$ given by

$$
M(p,|x|):=M_{\mathbf{c}, \sigma}(p,|x|)=\sup \left\{|p(\mathbf{c}, \sigma, \xi)| ;|\xi| \geq|x|, \xi \in \Sigma_{0}\left(x_{\infty}, \delta\right)\right\} .
$$

Suppose that $x_{\infty}>\varepsilon^{-1}$. Let $\mathfrak{A}=\mathfrak{A}\left(B_{0}, B_{x}, B_{*}, \Sigma_{0}\left(x_{\infty}, \delta\right), \varepsilon\right)(\subset \hat{\mathfrak{A}})$ be the family of $\phi \in \hat{\mathfrak{A}}$ such that $\|\phi\|_{\mathbf{c}, \sigma}(x, \eta)$ converges uniformly in $(\mathbf{c}, \sigma, x, \eta) \in B_{0} \times B_{x} \times B_{*} \times \Xi\left(\Sigma_{0}\left(x_{\infty}, \delta\right), \varepsilon\right)$, where

$$
\Xi\left(\Sigma_{0}\left(x_{\infty}, \delta\right), \varepsilon\right)=\bigcup_{x \in \Sigma_{0}\left(x_{\infty}, \delta\right)}\{x\} \times\left\{\eta ;\left|\eta x^{-1}\right|<\varepsilon,\left|\eta^{-1} x^{-1}\right|<\varepsilon\right\} .
$$

Let $D\left(B_{*}, \varepsilon, x_{\infty}, \delta\right)$ be as in Section 2 . Then, as shown below, the sum and the product are canonically defined in $\mathfrak{A}$. 


\section{Proposition 3.1.}

(1) Every $\phi \in \mathfrak{A}\left(B_{0}, B_{x}, B_{*}, \Sigma_{0}\left(x_{\infty}, \delta\right)\right.$, $\left.\varepsilon\right)$ is holomorphic in $(\mathbf{c}, \sigma, x) \in B_{0} \times B_{x} \times D\left(B_{*}, \varepsilon, x_{\infty}, \delta\right)$, and satisfies $|\phi(\mathbf{c}, \sigma, x)| \leq\|\phi\|_{\mathbf{c}, \sigma}\left(x, \mathrm{e}^{x} x^{\sigma}\right)$.

(2) Let $\phi, \psi \in \mathfrak{A}$. Then $\phi+\psi, \phi \psi \in \mathfrak{A}$, and $\|\phi+\psi\| \leq\|\phi\|+\|\psi\|$, $\|\phi \psi\| \leq\|\phi\|\|\psi\|$. If $a=a(\mathbf{c}, \sigma) \in \mathbb{Q}_{*}$, then $a \phi \in \mathfrak{A}$ and $\|a \phi\|=|a|\|\phi\|$.

Proof. Suppose that $\phi, \psi \in \mathfrak{A}$ are written as

$$
\begin{aligned}
& \phi=\sum_{n=1}^{\infty} p_{n}^{+}(x)\left(\mathrm{e}^{x} x^{\sigma-1}\right)^{n}+\sum_{n=1}^{\infty} p_{n}^{-}(x)\left(\mathrm{e}^{-x} x^{-\sigma-1}\right)^{n}+p_{0}(x) x^{-1}, \\
& \psi=\sum_{n=1}^{\infty} q_{n}^{+}(x)\left(\mathrm{e}^{x} x^{\sigma-1}\right)^{n}+\sum_{n=1}^{\infty} q_{n}^{-}(x)\left(\mathrm{e}^{-x} x^{-\sigma-1}\right)^{n}+q_{0}(x) x^{-1} .
\end{aligned}
$$

It is natural to set

$$
\phi \psi=\sum_{n=1}^{\infty} \varpi_{n}^{+}(x)\left(\mathrm{e}^{x} x^{\sigma-1}\right)^{n}+\sum_{n=1}^{\infty} \varpi_{n}^{-}(x)\left(\mathrm{e}^{-x} x^{-\sigma-1}\right)^{n}+\varpi_{0}(x) x^{-1},
$$

where each coefficient as a formal series is given by

$$
\begin{aligned}
& \varpi_{n}^{ \pm}(x)=\sum_{\nu=1}^{n-1} p_{\nu}^{ \pm}(x) q_{n-\nu}^{ \pm}(x)+x^{-1}\left(p_{n}^{ \pm}(x) q_{0}(x)+p_{0}(x) q_{n}^{ \pm}(x)\right)+\sum_{\nu=n+1}^{\infty} x^{-2(\nu-n)} \varpi_{n, \nu}^{ \pm}(x), \\
& \varpi_{0}(x)=x^{-2} p_{0}(x) q_{0}(x)+\sum_{\nu=1}^{\infty} x^{-2 \nu} \varpi_{\nu}^{0}(x)
\end{aligned}
$$

with $\varpi_{n, \nu}^{ \pm}(x)=p_{\nu}^{ \pm}(x) q_{\nu-n}^{\mp}(x)+p_{\nu-n}^{\mp}(x) q_{\nu}^{ \pm}(x), \varpi_{\nu}^{0}(x)=p_{\nu}^{+}(x) q_{\nu}^{-}(x)+p_{\nu}^{-}(x) q_{\nu}^{+}(x)$. If $(\mathbf{c}, \sigma, x) \in$ $B_{0} \times B_{x} \times B_{*} \times \Sigma_{0}\left(x_{\infty}, \delta\right)$, then, by the definition of $\|\cdot\|$, for $\left|\eta x^{-1}\right|,\left|\eta^{-1} x^{-1}\right|<\varepsilon$

$$
\begin{aligned}
\left|\varpi_{n, \nu}^{+}(x)\right| & \leq M\left(p_{\nu}^{+},|x|\right) M\left(q_{\nu-n}^{-},|x|\right)+M\left(p_{\nu-n}^{-},|x|\right) M\left(q_{\nu}^{+},|x|\right) \\
& \leq 2\|\phi\|(x, \eta)\|\psi\|(x, \eta)\left|\eta x^{-1}\right|^{-\nu}\left|\eta^{-1} x^{-1}\right|^{-(\nu-n)} \ll \varepsilon^{-2 \nu+n},
\end{aligned}
$$

and hence $\left|x^{-2(\nu-n)} \varpi_{n, \nu}^{+}(x)\right| \ll \varepsilon^{-n}(\varepsilon x)^{-2(\nu-n)}$, the implied constant not depending on $(\varepsilon, x)$. This implies that $\varpi_{n}^{+}(x)$ is holomorphic in $(\mathbf{c}, \sigma, x) \in B_{0} \times B_{x} \times B_{*} \times \Sigma_{0}\left(x_{\infty}, \delta\right)$. Furthermore, for a given integer $N \geq 1$, we have $\Sigma_{\nu \geq n+N}\left|x^{-2(\nu-n)} \varpi_{n, \nu}^{+}(x)\right| \ll \varepsilon^{-n}(\varepsilon x)^{-2 N}$ in the domain $B_{0} \times B_{x} \times B_{*} \times \Sigma_{0}\left(2 x_{\infty}, \delta\right)$, which implies that $\varpi_{n}^{+}(x)$ is represented by an asymptotic series in $\Sigma_{0}\left(x_{\infty}, \delta\right)$ uniformly in $(\mathbf{c}, \sigma) \in B_{0} \times B_{x} \times B_{*}$. Thus we have shown that $\phi \psi \in \hat{\mathfrak{A}}$. To evaluate $\|\phi \psi\|$ we note that, for $\nu>n \geq 1$,

$$
\begin{aligned}
& \left\|p_{\nu}^{+}(x)\left(\mathrm{e}^{x} x^{\sigma-1}\right)^{\nu} \cdot q_{\nu-n}^{-}(x)\left(\mathrm{e}^{-x} x^{-\sigma-1}\right)^{\nu-n}\right\|=\left\|\left(\mathrm{e}^{x} x^{\sigma-1}\right)^{n} p_{\nu}^{+}(x) q_{\nu-n}^{-}(x) x^{-2(\nu-n)}\right\| \\
& \quad=\left|\eta x^{-1}\right|^{n} \sup \left\{\left|p_{\nu}^{+}(\xi) q_{\nu-n}^{-}(\xi) \xi^{-2(\nu-n)}\right| ;|\xi| \geq|x|, \xi \in \Sigma_{0}\left(x_{\infty}, \delta\right)\right\} \\
& \quad \leq\left|\eta x^{-1}\right|^{\nu}\left|\eta^{-1} x\right|^{\nu-n}|x|^{-2(\nu-n)} \sup \left\{\left|p_{\nu}^{+}(\xi)\right| ; \ldots\right\} \sup \left\{\left|q_{\nu-n}^{-}(\xi)\right| ; \ldots\right\} \\
& \quad=\left|\eta x^{-1}\right|^{\nu} M\left(p_{\nu}^{+},|x|\right)\left|\eta^{-1} x^{-1}\right|^{\nu-n} M\left(q_{\nu-n}^{-},|x|\right) \\
& \quad=\left\|p_{\nu}^{+}(x)\left(\mathrm{e}^{x} x^{\sigma-1}\right)^{\nu}\right\|\left\|q_{\nu-n}^{-}(x)\left(\mathrm{e}^{-x} x^{-\sigma-1}\right)^{\nu-n}\right\|,
\end{aligned}
$$

and that, for $\nu \geq 1$,

$$
\begin{aligned}
& \left\|p_{\nu}^{+}(x)\left(\mathrm{e}^{x} x^{\sigma-1}\right)^{\nu} \cdot q_{0}(x) x^{-1}\right\|=\left|\eta x^{-1}\right|^{\nu} \sup \left\{\left|p_{\nu}^{+}(\xi) q_{0}(\xi) \xi^{-1}\right| ;|\xi| \geq|x|, \xi \in \Sigma_{0}\left(x_{\infty}, \delta\right)\right\} \\
& \quad \leq\left|\eta x^{-1}\right|^{\nu}|x|^{-1} \sup \left\{\left|p_{\nu}^{+}(\xi)\right| ; \ldots\right\} \sup \left\{\left|q_{0}(\xi)\right| ; \ldots\right\} \\
& \quad=\left\|p_{\nu}^{+}(x)\left(\mathrm{e}^{x} x^{\sigma-1}\right)^{\nu}\right\|\left\|q_{0}(x) x^{-1}\right\| .
\end{aligned}
$$

Using these inequalities we have $\|\phi \psi\| \leq\|\phi\|\|\psi\|$. 
Example 3.2. In the sector $|\arg x-\pi / 2|<\pi-\delta$, we may take a path $\gamma_{*}(x)(\ni \xi)$ starting from $x$ in such a way that $\left|\mathrm{e}^{-\xi} \mathrm{e}^{x}\right|$ or $\left|\mathrm{e}^{\xi} \mathrm{e}^{-x}\right|$ is monotone decreasing and decays exponentially along $\gamma_{*}(x)$. Then for $n \in \mathbb{N}$

$$
\begin{aligned}
& -\int_{\gamma_{*}(x)}\left(\mathrm{e}^{\xi} \xi^{\sigma-1}\right)^{n} \mathrm{~d} \xi=\left(\mathrm{e}^{x} x^{\sigma-1}\right)^{n} P_{n}^{(1)}(x), \\
& -\int_{\gamma_{*}(x)}\left(\mathrm{e}^{-\xi} \xi^{-\sigma-1}\right)^{n} \mathrm{~d} \xi=\left(\mathrm{e}^{-x} x^{-\sigma-1}\right)^{n} P_{n}^{(2)}(x),
\end{aligned}
$$

where $P_{n}^{(\iota)}(x) \sim \sum_{m=0}^{\infty} p_{n m}^{(\iota)}(\sigma) x^{-m}$ with $p_{n m}^{(\iota)}(\sigma) \in \mathbb{Q}[\sigma], \iota=1,2$, as $x \rightarrow \infty$ through the sector $|\arg x-\pi / 2|<\pi-\delta$. Furthermore if $g(x) \sim \sum_{m=0}^{\infty} g_{m} x^{-m}$ as $x \rightarrow \infty$ through this sector, we have

$$
-\int_{\gamma_{*}(x)}\left(\mathrm{e}^{\xi} \xi^{\sigma-1}\right)^{n} g(\xi) \mathrm{d} \xi=\left(\mathrm{e}^{x} x^{\sigma-1}\right)^{n} G_{n}(x), \quad G_{n}(x) \sim \sum_{m=0}^{\infty} G_{n m}(\sigma) x^{-m} .
$$

Clearly these integrals belong to $\mathfrak{A}$.

Proposition 3.3. Let $\left\{\phi_{k}\right\}_{k \geq 1} \subset \mathfrak{A}\left(B_{0}, B_{x}, B_{*}, \Sigma_{0}\left(x_{\infty}, \delta\right), \varepsilon\right)$ with

$$
\phi_{k}=\sum_{n=1}^{\infty} p_{n}^{(k)+}(x)\left(\mathrm{e}^{x} x^{\sigma-1}\right)^{n}+\sum_{n=1}^{\infty} p_{n}^{(k)-}(x)\left(\mathrm{e}^{-x} x^{-\sigma-1}\right)^{n}+p_{0}^{(k)}(x) x^{-1}
$$

be such that $\left\|\phi_{k}\right\| \ll \tilde{\varepsilon}^{k-N}|x|^{-N+k_{0}}$ for every pair of integers $(k, N)$ with $1 \leq k_{0} \leq N \leq k$ if $\left|\eta x^{-1}\right|<\varepsilon,\left|\eta^{-1} x^{-1}\right|<\varepsilon,|x|>x_{\infty}$, where $k_{0}$ is a given positive integer, $\tilde{\varepsilon}$ is some number satisfying $0<\tilde{\varepsilon}<1$, and the implied constant does not depend on $k$. Then, $\phi^{\infty}=\sum_{k=1}^{\infty} \phi_{k}$ also belongs to $\mathfrak{A}\left(B_{0}, B_{x}, B_{*}, \Sigma_{0}\left(x_{\infty}, \delta\right), \varepsilon\right)$ and $\phi^{\infty}$ is given by

$$
\phi^{\infty}=\sum_{n=1}^{\infty} p_{n}^{\infty+}(x)\left(\mathrm{e}^{x} x^{\sigma-1}\right)^{n}+\sum_{n=1}^{\infty} p_{n}^{\infty-}(x)\left(\mathrm{e}^{-x} x^{-\sigma-1}\right)^{n}+p_{0}^{\infty}(x) x^{-1}
$$

with $p_{n}^{\infty \pm}(x)=\sum_{k=1}^{\infty} p_{n}^{(k) \pm}(x)=[1], p_{0}^{\infty}(x)=\sum_{k=1}^{\infty} p_{0}^{(k)}(x)=[1]$

Proof. By the condition with $N=k_{0}, \sum_{k=k_{0}}^{\infty} \phi_{k}$ as a double series converges uniformly and absolutely, and hence $p_{n}^{\infty \pm}(x)=\sum_{k=1}^{\infty} p_{n}^{(k) \pm}(x), p_{0}^{\infty}(x)=\sum_{k=1}^{\infty} p_{0}^{(k)}(x)$ are holomorphic in $\Sigma_{0}\left(x_{\infty}, \delta\right)$. It is sufficient to show that $\phi^{\infty} \in \hat{\mathfrak{A}}\left(B_{0}, B_{x}, B_{*}, \Sigma_{0}\left(x_{\infty}, \delta\right), \varepsilon\right)$, that is, $p_{n}^{\infty \pm}(x)$ and $p_{0}^{\infty}(x)$ may be represented by asymptotic series. For a given positive number $N \geq k_{0}$, if $k \geq N$ and if $\left|\eta x^{-1}\right|<\varepsilon,\left|\eta^{-1} x^{-1}\right|<\varepsilon,|x|>x_{\infty}>\varepsilon^{-1}$, then

$$
M\left(p_{n}^{(k)+},|x|\right) \leq\left\|\phi_{k}\right\|(x, \eta)\left|\eta x^{-1}\right|^{-n} \ll\left|\eta x^{-1}\right|^{-n} \tilde{\varepsilon}^{k-N}|x|^{-N+k_{0}},
$$

the implied constant possibly depending on $N$ only. For $x \in \Sigma_{0}\left(x_{\infty}, \delta\right)$, letting $\eta \rightarrow \varepsilon x$, we have $\left|p_{n}^{(k)+}(x)\right| \ll \varepsilon^{-n} \tilde{\varepsilon}^{k-N}|x|^{-N+k_{0}}$, which means $\left|\sum_{k=N}^{\infty} p_{n}^{(k)+}(x)\right| \ll \varepsilon^{-n}(1-\tilde{\varepsilon})^{-1}|x|^{-N+k_{0}}$ in $\Sigma_{0}\left(x_{\infty}, \delta\right)$. Substitution of this and $p_{n}^{(k)+}(x)$ with $k \leq N-1$ into $p_{n}^{\infty+}(x)$ yields the asymptotic representation of $p_{n}^{\infty+}(x)$. Thus we obtain the proposition. 
Remark 3.4. Under the supposition of Proposition 3.3, for $p_{n}^{(k) \pm}(x) \sim \sum_{m=0}^{\infty} p_{n m}^{(k) \pm} x^{-m}$ and $p_{0}^{(k)}(x) \sim \sum_{m=0}^{\infty} p_{0 m}^{(k)} x^{-m}$, the asymptotic representations of $p_{n}^{\infty \pm}(x)$ and $p_{0}^{\infty}(x)$ are written in the form $p_{n}^{\infty \pm}(x) \sim \sum_{m=0}^{\infty} p_{n m}^{\infty \pm} x^{-m}$ and $p_{0}^{\infty}(x) \sim \sum_{m=0}^{\infty} p_{0 m}^{\infty} x^{-m}$ with coefficients $p_{n m}^{\infty \pm}=\sum_{k=1}^{m+k_{0}} p_{n m}^{(k) \pm}$ and $p_{0 m}^{\infty}=\sum_{k=1}^{m+k_{0}} p_{0 m}^{(k)}$, respectively.

The following sums of the form $\sum_{k=1}^{\infty} \phi^{k}$ belong to $\mathfrak{A}\left(B_{0}, B_{x}, B_{*}, \Sigma_{0}\left(x_{\infty}, \delta\right), \varepsilon\right)$, although $\left\|\phi^{k}\right\|$ does not necessarily satisfy the condition of Proposition 3.3.

Example 3.5. For $\phi=p^{+}(x) \mathrm{e}^{x} x^{\sigma-1}+p^{-}(x) \mathrm{e}^{-x} x^{-\sigma-1} \in \mathfrak{A}\left(B_{0}, B_{x}, B_{*}, \Sigma_{0}\left(x_{\infty}, \delta\right), \varepsilon\right)$, if $\varepsilon^{\prime}<\varepsilon$ is sufficiently small, then $\sum_{k=1}^{\infty} \phi^{k} \in \mathfrak{A}\left(B_{0}, B_{x}, B_{*}, \Sigma_{0}\left(x_{\infty}, \delta\right), \varepsilon^{\prime}\right)$.

Verification. Suppose that $p^{ \pm}(x) \sim p_{0}^{ \pm}+\sum_{m=1}^{\infty} p_{m}^{ \pm} x^{-m}$ and that $\left|p^{ \pm}(x)-p_{0}^{ \pm}\right|<r_{0}$ for $(\mathbf{c}, \sigma, x) \in B_{0} \times B_{x} \times B_{*} \times \Sigma_{0}\left(x_{\infty}, \delta\right)$. We may choose $\varepsilon^{\prime}$ in such a way that $\sum_{k=1}^{\infty}\left(\left(\left|p_{0}^{+}\right|+\left|\xi_{+}\right|\right)\left|\eta_{+}\right|+\right.$ $\left.\left(\left|p_{0}^{-}\right|+\left|\xi_{-}\right|\right)\left|\eta_{-}\right|\right)^{k}$ is convergent for $\left(\mathbf{c}, \sigma, \xi_{+}, \xi_{-}, \eta_{+}, \eta_{-}\right)$satisfying $(\mathbf{c}, \sigma) \in B_{0} \times B_{x} \times B_{*},\left|\xi_{ \pm}\right|<$ $2 r_{0},\left|\eta_{ \pm}\right|<\varepsilon^{\prime}$. Then $\sum_{k=1}^{\infty}\left(\left(p_{0}^{+}+\xi_{+}\right) \eta x^{-1}+\left(p_{0}^{-}+\xi_{-}\right) \eta^{-1} x^{-1}\right)^{k}$ converges absolutely for $\left(\mathbf{c}, \sigma, \xi_{+}, \xi_{-}\right)$ as above and for $(\eta, x)$ satisfying $\left|\eta x^{-1}\right|,\left|\eta^{-1} x^{-1}\right|<\varepsilon^{\prime},|x|>1 / \varepsilon^{\prime}$, and is written in the form

$$
\sum_{n=1}^{\infty}\left(\pi_{n}^{+}\left(\xi_{+}, \xi_{-}, x^{-1}\right)\left(\eta x^{-1}\right)^{n}+\pi_{n}^{-}\left(\xi_{+}, \xi_{-}, x^{-1}\right)\left(\eta^{-1} x^{-1}\right)^{n}\right)+\pi_{0}\left(\xi_{+}, \xi_{-}, x^{-1}\right) x^{-1}
$$

where $\pi_{n}^{ \pm}\left(\xi_{+}, \xi_{-}, x^{-1}\right)=\sum_{m=0}^{\infty} \pi_{n m}^{ \pm}\left(\xi_{+}, \xi_{-}\right) x^{-m}, \pi_{0}\left(\xi_{+}, \xi_{-}, x^{-1}\right)=\sum_{m=0}^{\infty} \pi_{0 m}\left(\xi_{+}, \xi_{-}\right) x^{-m}$ with $\pi_{n m}^{ \pm}\left(\xi_{+}, \xi_{-}\right)=\sum_{k_{1}, k_{2}} \pi_{n m k_{1} k_{2}}^{ \pm} \xi_{+}^{k_{1}} \xi_{-}^{k_{2}}, \pi_{0 m}\left(\xi_{+}, \xi_{-}\right)=\sum_{k_{1}, k_{2}} \pi_{0 m k_{1} k_{2}} \xi_{+}^{k_{1}} \xi_{-}^{k_{2}}$ converge for $\left|\xi_{ \pm}\right|<2 r_{0}$, $|x|>1 / \varepsilon^{\prime}$. Inserting $\eta=\mathrm{e}^{x} x^{\sigma}, \xi_{ \pm}=p^{ \pm}(x)-p_{0}^{ \pm} \sim \sum_{m=1}^{\infty} p_{m}^{ \pm} x^{-m}$ into this we have the conclusion.

Example 3.6. For $\phi=p_{1}(x) \mathrm{e}^{x} x^{\sigma-1}+p_{2}(x)\left(\mathrm{e}^{x} x^{\sigma-1}\right)^{2} \in \mathfrak{A}\left(B_{0}, B_{x}, B_{*}, \Sigma_{0}\left(x_{\infty}, \delta\right), \varepsilon\right)$, we have $\sum_{k=1}^{\infty} \phi^{k} \in \mathfrak{A}\left(B_{0}, B_{x}, B_{*}, \Sigma_{0}\left(x_{\infty}, \delta\right), \varepsilon\right)$, because, for each $n \geq 1$, the coefficient of $\left(\mathrm{e}^{x} x^{\sigma-1}\right)^{n}$ is a finite sum of asymptotic series in $x^{-1}$.

The following lemma is used in evaluating the primitive function of $x^{-1} \phi$.

Lemma 3.7. For every $(\sigma, x) \in B_{*} \times \Sigma_{0}\left(\tilde{x}_{\infty}, \delta\right)$,

$$
\left|\mathrm{e}^{x} x^{\sigma-1}\right|^{-n} \int_{\gamma(x)}\left|\mathrm{e}^{\xi} \xi^{\sigma-1}\right|^{n} \frac{|\mathrm{d} \xi|}{|\xi|},\left|\mathrm{e}^{-x} x^{-\sigma-1}\right|^{-n} \int_{\gamma(x)}\left|\mathrm{e}^{-\xi} \xi^{-\sigma-1}\right|^{n} \frac{|\mathrm{d} \xi|}{|\xi|} \leq \frac{1}{n}\left(1+\frac{2}{\sin \delta}\right),
$$

$n=1,2,3, \ldots$ Here $\tilde{x}_{\infty}=\tilde{x}_{\infty}\left(B_{*}, \delta\right)$ is a sufficiently large number depending on $\left(B_{*}, \delta\right)$, and $\gamma(x)$ is a path with the properties:

(i) $\gamma(x)$ starts from $x$ and tends to $\infty \mathrm{e}^{\pi \mathrm{i} / 2}$, and $\xi \in \gamma(x)$ is given by $\xi=\mathrm{i} \tau \exp (-\mathrm{i} \theta(\tau))$ with $|x| \leq \tau<\infty$, where $\theta(\tau)$ satisfies $\theta(|x|)=\pi / 2-\arg x, \theta(\tau) \rightarrow 0$ as $\tau \rightarrow \infty$;

(ii) for every $\xi \in \gamma(x),\left|\mathrm{e}^{x} x^{\sigma}\right|=\left|\mathrm{e}^{\xi} \xi^{\sigma}\right|$;

(iii) for every $\xi \in \gamma(x),|\xi| \geq|x|$, and $|\pi / 2-\arg \xi|<\pi / 2-\delta$. 
Proof. We substitute $\xi=\mathrm{i} \tau \exp (-\mathrm{i} \theta(\tau))=\tau \exp (\mathrm{i}(\pi / 2-\theta(\tau)))$ into $\left|\mathrm{e}^{x} x^{\sigma}\right|=\left|\mathrm{e}^{\xi} \xi^{\sigma}\right|$ to obtain

$$
\operatorname{Re} x+\operatorname{Re} \sigma \cdot \log |x|-\operatorname{Im} \sigma \cdot \arg x=\tau \sin \theta(\tau)+\operatorname{Re} \sigma \cdot \log \tau-\operatorname{Im} \sigma \cdot(\pi / 2-\theta(\tau)) .
$$

Choose $\tilde{x}_{\infty}=\tilde{x}_{\infty}\left(B_{*}, \delta\right)$ in such a way that, for every $\sigma \in B_{*}$,

$$
\tilde{x}_{\infty}>100(|\sigma|+1)(\tan \delta+1 / \sin \delta)>200(|\sigma|+1) .
$$

Then, for any $x \in \Sigma_{0}\left(\tilde{x}_{\infty}, \delta\right)$, the function $\theta(\tau)$ satisfying (3.1) and $\theta(|x|)=\pi / 2-\arg x$ is uniquely determined near $\tau=|x|$ by the implicit function theorem. Note that

$$
\begin{aligned}
& \theta^{\prime}(\tau)=-\frac{\sin \theta(\tau)+\operatorname{Re} \sigma \cdot \tau^{-1}}{\tau \cos \theta(\tau)+\operatorname{Im} \sigma}, \\
& (\operatorname{Im} \xi(\tau))^{\prime}=\cos \theta(\tau)-\tau \theta^{\prime}(\tau) \sin \theta(\tau)=\frac{\tau+\operatorname{Im} \sigma \cdot \cos \theta(\tau)+\operatorname{Re} \sigma \cdot \sin \theta(\tau)}{\tau \cos \theta(\tau)+\operatorname{Im} \sigma}, \\
& (\operatorname{Re} \xi(\tau))^{\prime}=\sin \theta(\tau)+\tau \theta^{\prime}(\tau) \cos \theta(\tau)=\frac{\operatorname{Im} \sigma \cdot \sin \theta(\tau)-\operatorname{Re} \sigma \cdot \cos \theta(\tau)}{\tau \cos \theta(\tau)+\operatorname{Im} \sigma} .
\end{aligned}
$$

As long as $\tau \geq|x|>\tilde{x}_{\infty},|\theta(\tau)|<\pi / 2-\delta$, from (3.2), (3.4) and (3.5) it follows that

$$
\begin{gathered}
(\operatorname{Im} \xi(\tau))^{\prime} \geq \frac{\tau(1-1 / 100)}{\tau(1+1 / 100) \sin \delta} \geq \frac{1}{2 \sin \delta}>0, \\
\left|(\operatorname{Re} \xi(\tau))^{\prime}\right| \leq \frac{2|\sigma|}{\tau(1-1 / 100) \sin \delta}<\frac{3(|\sigma|+1)}{\tau \sin \delta},
\end{gathered}
$$

and hence $(\operatorname{Im} \xi(\tau))^{\prime} /\left|(\operatorname{Re} \xi(\tau))^{\prime}\right|>\tau(|\sigma|+1)^{-1} / 6>10 \tan \delta$. This fact implies that $\theta(\tau)$ may be prolonged for $\tau \geq|x|>\tilde{x}_{\infty}$ and that (ii) and (iii) are fulfilled. Then, by (3.3) with (3.2),

$$
\left|\theta^{\prime}(\tau)\right| \leq \frac{1+1 / 3}{\tau(1-1 / 3) \cos \theta(\tau)} \leq \frac{2 \tau^{-1}}{\sin \delta}
$$

and hence $|\mathrm{d} \xi|=|\mathrm{d} \xi / \mathrm{d} \tau| \mathrm{d} \tau \leq\left|\mathrm{ie}^{-\mathrm{i} \theta(\tau)}+\theta^{\prime}(\tau) \tau \mathrm{e}^{-\mathrm{i} \theta(\tau)}\right| \mathrm{d} \tau \leq\left(1+\left|\tau \theta^{\prime}(\tau)\right|\right) \mathrm{d} \tau \leq(1+2 / \sin \delta) \mathrm{d} \tau$. Using this and (ii) we obtain

$$
\left|\mathrm{e}^{x} x^{\sigma-1}\right|^{-n} \int_{\gamma(x)}\left|\mathrm{e}^{\xi} \xi^{\sigma-1}\right|^{n} \frac{|\mathrm{d} \xi|}{|\xi|}=|x|^{n} \int_{|x|}^{\infty} \tau^{-n-1}\left(1+\frac{2}{\sin \delta}\right) \mathrm{d} \tau \leq \frac{1}{n}\left(1+\frac{2}{\sin \delta}\right) .
$$

This completes the proof.

Remark 3.8. If $|\arg x-3 \pi / 2|<\pi / 2-\delta,|x|>\tilde{x}_{\infty}^{\prime}\left(B_{*}, \delta\right)$, then along the path $\gamma_{3 \pi / 2}(x)$ defined by $\xi=\mathrm{i} \tau \exp \left(-\mathrm{i} \theta_{3 \pi / 2}(\tau)\right)$ with $\theta_{3 \pi / 2}(\tau)=-\pi+\theta(\tau)$ (cf. (3.1)) the same estimates for the integrals are obtained. If $|\arg x+\pi / 2|<\pi / 2-\delta$, then $\gamma_{-\pi / 2}(x)$ given by $\xi=\mathrm{i} \tau \exp \left(-\mathrm{i} \theta_{-\pi / 2}(\tau)\right)$ with $\theta_{-\pi / 2}(\tau)=\pi+\theta(\tau)$ has the same property. These paths are obtained by making the substitutions $(x, \xi, \sigma) \mapsto\left(x \mathrm{e}^{-\pi \mathrm{i}}, \xi \mathrm{e}^{-\pi \mathrm{i}},-\sigma\right)$ and $(x, \xi, \sigma) \mapsto\left(x \mathrm{e}^{\pi \mathrm{i}}, \xi \mathrm{e}^{\pi \mathrm{i}},-\sigma\right)$, respectively, in the definition of $\gamma(x)$.

Furthermore we have

Lemma 3.9. For every $\sigma \in B_{*}$

$$
\left|\mathrm{e}^{x} x^{\sigma}\right|^{-n} \int_{\gamma_{\pi}(x)}\left|\mathrm{e}^{\xi} \xi^{\sigma}\right|^{n}|\mathrm{~d} \xi| \leq \frac{2}{n|\cos (\arg x)|}, \quad n=1,2,3, \ldots,
$$

in the sector $|\arg x-\pi|<\pi / 2-\delta,|x|>\tilde{x}_{\infty}^{\prime \prime}\left(B_{*}, \delta\right)$, where $\tilde{x}_{\infty}^{\prime \prime}\left(B_{*}, \delta\right)$ is sufficiently large and $\gamma_{\pi}(x)$ is a ray starting from $x$ and tending to $\infty \mathrm{e}^{\mathrm{i} \text { arg } x}$. 
Proof. The path $\gamma_{\pi}(x)$ is given by $\xi=\xi(t)=x+t \mathrm{e}^{\mathrm{i} \arg x}, 0 \leq t<\infty$. Then

$$
\left|\mathrm{e}^{\xi} \xi^{\sigma}\right|^{n}\left|\mathrm{e}^{x} x^{\sigma}\right|^{-n}=\exp (-r(t)), \quad-r(t)=n(t \cos (\arg x)+\operatorname{Re} \sigma \cdot \log (1+t /|x|)) .
$$

Since $-\mathrm{d} r / \mathrm{d} t=n(\cos (\arg x)+\operatorname{Re} \sigma /(|x|+t))$, we have $\mathrm{d} t / \mathrm{d} r \leq 2 /(n|\cos (\arg x)|)$, if $\tilde{x}_{\infty}^{\prime \prime}\left(B_{*}, \delta\right)$ is sufficiently large. Hence

$$
\left|\mathrm{e}^{x} x^{\sigma}\right|^{-n} \int_{\gamma_{\pi}(x)}\left|\mathrm{e}^{\xi} \xi^{\sigma}\right|^{n}|\mathrm{~d} \xi|=\int_{0}^{\infty} \mathrm{e}^{-r(t)} \frac{\mathrm{d} t}{\mathrm{~d} r} \mathrm{~d} r \leq \frac{2}{n|\cos (\arg x)|} \int_{0}^{\infty} \mathrm{e}^{-r} \mathrm{~d} r \leq \frac{2}{n|\cos (\arg x)|},
$$

which completes the proof.

Remark 3.10. Similarly, for $\sigma \in B_{*}$, in the sector $|\arg x|<\pi / 2-\delta,|x|>\tilde{x}_{\infty}^{\prime \prime}\left(B_{*}, \delta\right)$, we have

$$
\left|\mathrm{e}^{-x} x^{-\sigma}\right|^{-n} \int_{\gamma_{0}(x)}\left|\mathrm{e}^{-\xi} \xi^{-\sigma}\right|^{n}|\mathrm{~d} \xi| \leq \frac{2}{n \cos (\arg x)},
$$

where $\gamma_{0}(x)$ is a ray starting from $x$ and tending to $\infty \mathrm{e}^{\mathrm{i} \arg x}$.

Lemma 3.11. Let $B_{*}, \tilde{x}_{\infty}$ and $\gamma(x)$ be as in Lemma 3.7 and let $x_{\infty}>\tilde{x}_{\infty}$. Suppose that $p(x)$ is holomorphic in $(\mathbf{c}, \sigma, x) \in B_{0} \times B_{x} \times B_{*} \times \Sigma_{0}\left(x_{\infty}, \delta\right)$ and admits the asymptotic representation $p(x) \sim \sum_{m=0}^{\infty} p_{m} x^{-m}, p_{m} \in \mathbb{Q}_{*}$ uniformly in $(\mathbf{c}, \sigma) \in B_{0} \times B_{x} \times B_{*}$ as $x \rightarrow \infty$ through $\Sigma_{0}\left(x_{\infty}, \delta\right)$. Then, for any $n \in \mathbb{N}$,

$$
\begin{aligned}
& I_{\gamma(x)}^{+n}(p(x)):=-\left(\mathrm{e}^{x} x^{\sigma-1}\right)^{-n} \int_{\gamma(x)}\left(\mathrm{e}^{\xi} \xi^{\sigma-1}\right)^{n} p(\xi) \mathrm{d} \xi \\
& I_{\gamma(x)}^{-n}(p(x)):=-\left(\mathrm{e}^{-x} x^{-\sigma-1}\right)^{-n} \int_{\gamma(x)}\left(\mathrm{e}^{-\xi} \xi^{-\sigma-1}\right)^{n} p(\xi) \mathrm{d} \xi
\end{aligned}
$$

are holomorphic in $(\mathbf{c}, \sigma, x)$ and admit the asymptotic representations

$$
I_{\gamma(x)}^{+n}(p(x)) \sim \sum_{m=0}^{\infty} P_{n m}^{+} x^{-m}, \quad I_{\gamma(x)}^{-n}(p(x)) \sim \sum_{m=0}^{\infty} P_{n m}^{-} x^{-m}
$$

with $P_{n m}^{ \pm} \in \mathbb{Q}_{*}$ uniformly in $(\mathbf{c}, \sigma) \in B_{0} \times B_{x} \times B_{*}$ as $x \rightarrow \infty$ through $\Sigma_{0}\left(x_{\infty}, \delta\right)$. Furthermore, if $p(x)=O\left(x^{-1}\right)$,

$$
I_{\gamma(x)}^{0}(p(x)):=-x \int_{\gamma(x)} \xi^{-1} p(\xi) \mathrm{d} \xi \sim \sum_{m=0}^{\infty} P_{n m}^{0} x^{-m}
$$

with $P_{n m}^{0} \in \mathbb{Q}_{*}$.

Remark 3.12. The integrals $I_{\gamma(x)}^{ \pm 1}(p(x))$ are not necessarily absolutely convergent.

Remark 3.13. If $p(x) \sim \sum_{m=0}^{\infty} p_{m} x^{-m}$ in the sector $|\arg x-3 \pi / 2|<\pi / 2-\delta$, then

$$
I_{\gamma_{3 \pi / 2}(x)}^{ \pm n}(p(x)):=-\left(\mathrm{e}^{ \pm x} x^{ \pm \sigma-1}\right)^{-n} \int_{\gamma_{3 \pi / 2}(x)}\left(\mathrm{e}^{ \pm \xi} \xi^{ \pm \sigma-1}\right)^{n} p(\xi) \mathrm{d} \xi
$$

with $\gamma_{3 \pi / 2}(x)$ in Remark 3.8 admit asymptotic representations of the same form as above. Furthermore, for $p(x)$ in the sector $|\arg x+\pi / 2|<\pi / 2-\delta$, we may define $I_{\gamma_{-\pi / 2}(x)}^{ \pm n}(p(x))$ with $\gamma_{-\pi / 2}(x)$ as in Remark 3.8 having the same property. 
Proof of Lemma 3.11. For every $(k, n) \in(\mathbb{N} \cup\{0\}) \times \mathbb{N}$, integrating by parts and using (ii) of Lemma 3.7, we have

$$
\begin{aligned}
I_{n, k}(x):= & \left(\mathrm{e}^{x} x^{\sigma-1}\right)^{-n} \int_{\gamma(x)}\left(\mathrm{e}^{\xi} \xi^{\sigma-1}\right)^{n} \xi^{-k} \mathrm{~d} \xi=\left[\mathrm{e}^{-n x} x^{-n(\sigma-1)} \frac{\mathrm{e}^{n \xi}}{n} \xi^{n(\sigma-1)-k}\right]_{\gamma(x)} \\
& -\frac{n(\sigma-1)-k}{n}\left(\mathrm{e}^{x} x^{\sigma-1}\right)^{-n} \int_{\gamma(x)}\left(\mathrm{e}^{\xi} \xi^{\sigma-1}\right)^{n} \xi^{-k-1} \mathrm{~d} \xi \\
= & -\frac{x^{-k}}{n}-\frac{n(\sigma-1)-k}{n} I_{n, k+1}(x)
\end{aligned}
$$

in $\Sigma_{0}\left(x_{\infty}, \delta\right)$, because $\left(\left|\mathrm{e}^{\xi} \xi^{\sigma}\right| /\left|\mathrm{e}^{x} x^{\sigma}\right|\right)^{n}|x|^{n}|\xi|^{-n-k} \rightarrow 0$ as $\xi \rightarrow \infty$ along $\gamma(x)$. Furthermore, $I_{n, k+1}(x)$ converges absolutely. If $k \geq 1$, then, by (iii) of Lemma 3.7,

$$
\left|I_{n, k}(x)\right| \leq \int_{\gamma(x)}\left|\mathrm{e}^{x} x^{\sigma-1}\right|^{-n}\left|\mathrm{e}^{\xi} \xi^{\sigma-1}\right|^{n}|x|^{-(k-1)} \frac{|\mathrm{d} \xi|}{|\xi|} \ll|x|^{-(k-1)} .
$$

Similarly, if $g(\xi) \ll|\xi|^{-k}$,

$$
\left|\int_{\gamma(x)}\left(\mathrm{e}^{x} x^{\sigma-1}\right)^{-n}\left(\mathrm{e}^{\xi} \xi^{\sigma-1}\right)^{n} g(\xi) \mathrm{d} \xi\right| \ll|x|^{-(k-1)} .
$$

Combining these facts suitably, we may show that $I_{\gamma(x)}^{+n}(p(x))$ is holomorphic in $(\mathbf{c}, \sigma, x)$ and get the asymptotic expansion of $I_{\gamma(x)}^{+n}(p(x))$ as in the lemma.

Now we are ready to define the primitive function of $\phi \in \hat{\mathfrak{A}}$ or $\mathfrak{A}$. Let $x_{\infty} \geq \tilde{x}_{\infty}$ with $\tilde{x}_{\infty}$ as in Lemma 3.7. Suppose that

$$
\begin{aligned}
\phi= & \sum_{n=1}^{\infty} p_{n}^{+}(x)\left(\mathrm{e}^{x} x^{\sigma-1}\right)^{n}+\sum_{n=1}^{\infty} p_{n}^{-}(x)\left(\mathrm{e}^{-x} x^{-\sigma-1}\right)^{n}+p_{0}(x) x^{-1} \\
& \in \hat{\mathfrak{A}}=\hat{\mathfrak{A}}\left(B_{0}, B_{x}, B_{*}, \Sigma_{0}\left(x_{\infty}, \delta\right), \varepsilon\right)
\end{aligned}
$$

and that $p_{0}(x)=O\left(x^{-1}\right)$. Let $\gamma(x)$ be as in Lemma 3.7, and $I_{\gamma(x)}^{ \pm n}(\cdot), I_{\gamma(x)}^{0}(\cdot)$ as in Lemma 3.11. Then we define

$$
\mathcal{I}[\phi]:=\sum_{n=1}^{\infty} P_{n}^{+}(x)\left(\mathrm{e}^{x} x^{\sigma-1}\right)^{n}+\sum_{n=1}^{\infty} P_{n}^{-}(x)\left(\mathrm{e}^{-x} x^{-\sigma-1}\right)^{n}+P_{0}(x) x^{-1}
$$

with

$$
P_{n}^{+}(x)=I_{\gamma(x)}^{+n}\left(p_{n}^{+}(x)\right), \quad P_{n}^{-}(x)=I_{\gamma(x)}^{-n}\left(p_{n}^{-}(x)\right), \quad P_{0}(x)=I_{\gamma(x)}^{0}\left(p_{0}(x)\right) .
$$

By Lemma 3.11, $P_{n}^{ \pm}(x)$ and $P_{0}(x)$ are represented by asymptotic series of the form

$$
P_{n}^{ \pm}(x) \sim \sum_{m=0}^{\infty} P_{n m}^{ \pm} x^{-m}, \quad P_{0}(x) \sim \sum_{m=0}^{\infty} P_{0 m} x^{-m}
$$

with $P_{n m}^{ \pm}, P_{0 m} \in \mathbb{Q}_{*}$ uniformly in $(\mathbf{c}, \sigma) \in B_{0} \times B_{x} \times B_{*}$ as $x \rightarrow \infty$ through $\Sigma_{0}\left(x_{\infty}, \delta\right)$, and hence $\mathcal{I}[\phi] \in \mathfrak{A}$. The series $\mathcal{I}[\phi]$ is a formal primitive function of $\phi$.

Proposition 3.14. Suppose that $\phi \in \hat{\mathfrak{A}}$ and $p_{0}(x)=O\left(x^{-1}\right)$. Then $(\mathrm{d} / \mathrm{d} x) \mathcal{I}[\phi]=\phi$ as a formal series. 
Proof. For $n \geq 1$, let $p_{n 0}^{+}=\lim _{x \rightarrow \infty} p_{n}^{+}(x)$. Then

$$
I_{\gamma(x)}^{+n}\left(p_{n}^{+}(x)\right)=I_{\gamma(x)}^{+n}\left(p_{n}^{+}(x)-p_{n 0}^{+}\right)+p_{n 0}^{+} I_{\gamma(x)}^{+n}(1) .
$$

Since $\left(\mathrm{e}^{x} x^{\sigma-1}\right)^{-n}\left(\mathrm{e}^{\xi} \xi^{\sigma-1}\right)^{n}\left(p_{n}^{+}(\xi)-p_{n 0}^{+}\right) \ll|x||\xi|^{-2}$ as $\xi \rightarrow \infty$ along $\gamma(x), I_{\gamma(x)}^{+n}\left(p_{n}^{+}(x)-p_{n 0}^{+}\right)$ converges absolutely, and hence

$$
(\mathrm{d} / \mathrm{d} x)\left(\left(\mathrm{e}^{x} x^{\sigma-1}\right)^{n} I_{\gamma(x)}^{+n}\left(p_{n}^{+}(x)-p_{n 0}^{+}\right)\right)=\left(\mathrm{e}^{x} x^{\sigma-1}\right)^{n}\left(p_{n}^{+}(x)-p_{n 0}^{+}\right) .
$$

As shown in the proof of Lemma 3.11, $I_{\gamma(x)}^{+n}(1)=1 / n-(\sigma-1) I_{\gamma(x)}^{+n}\left(x^{-1}\right)$, the last integral being absolutely convergent. This implies

$$
(\mathrm{d} / \mathrm{d} x)\left(\left(\mathrm{e}^{x} x^{\sigma-1}\right)^{n} I_{\gamma(x)}^{+n}(1) p_{n 0}^{+}\right)=\left(\mathrm{e}^{x} x^{\sigma-1}\right)^{n} p_{n 0}^{+} .
$$

Thus we obtain the conclusion.

Furthermore we have

Proposition 3.15. If $\phi \in \mathfrak{A}=\mathfrak{A}\left(B_{0}, B_{x}, B_{*}, \Sigma_{0}\left(x_{\infty}, \delta\right), \varepsilon\right)$, then $\mathcal{I}\left[x^{-1} \phi\right] \in \mathfrak{A}$ and $\left\|\mathcal{I}\left[x^{-1} \phi\right]\right\| \leq$ $(1+2 / \sin \delta)\|\phi\|$.

Proof. By definition

$$
\mathcal{I}\left[x^{-1} \phi\right]=\sum_{n=1}^{\infty} \hat{P}_{n}^{+}(x)\left(\mathrm{e}^{x} x^{\sigma-1}\right)^{n}+\sum_{n=1}^{\infty} \hat{P}_{n}^{-}(x)\left(\mathrm{e}^{-x} x^{-\sigma-1}\right)^{n}+\hat{P}_{0}(x) x^{-1} \in \hat{\mathfrak{A}},
$$

where

$$
\begin{aligned}
\hat{P}_{n}^{ \pm}(x) & =I_{\gamma(x)}^{ \pm n}\left(x^{-1} p_{n}^{ \pm}(x)\right) \\
& =-\left(\mathrm{e}^{ \pm x} x^{ \pm \sigma-1}\right)^{-n} \int_{\gamma(x)}\left(\mathrm{e}^{ \pm \xi} \xi^{ \pm \sigma-1}\right)^{n} \xi^{-1} p_{n}^{ \pm}(\xi) \mathrm{d} \xi \sim \hat{P}_{n 1}^{ \pm} x^{-1}+\cdots, \\
\hat{P}_{0}(x) & =I_{\gamma(x)}^{0}\left(x^{-1} p_{0}(x)\right)=-x \int_{\gamma(x)} \xi^{-2} p_{0}(\xi) \mathrm{d} \xi \sim \hat{P}_{00}+\cdots
\end{aligned}
$$

By Lemma 3.7, for any $x, \tilde{x} \in \Sigma_{0}\left(x_{\infty}, \delta\right)$ such that $|\tilde{x}| \geq|x|$,

$$
\begin{aligned}
\left|\hat{P}_{n}^{ \pm}(\tilde{x})\right| & \leq \int_{\gamma(\tilde{x})}\left|\mathrm{e}^{ \pm \tilde{x}} \tilde{x}^{ \pm \sigma-1}\right|^{-n}\left|\mathrm{e}^{ \pm \xi} \xi^{ \pm \sigma-1}\right|^{n}|\xi|^{-1} M\left(p_{n}^{ \pm},|\tilde{x}|\right)|\mathrm{d} \xi| \\
& \leq(1+2 / \sin \delta) M\left(p_{n}^{ \pm},|\tilde{x}|\right) \leq(1+2 / \sin \delta) M\left(p_{n}^{ \pm},|x|\right),
\end{aligned}
$$

which implies $M\left(\hat{P}_{n}^{ \pm},|x|\right) \leq(1+2 / \sin \delta) M\left(p_{n}^{ \pm},|x|\right)$. Similarly, we have $M\left(\hat{P}_{0},|x|\right) \leq(1+$ $2 / \sin \delta) M\left(p_{0},|x|\right)$. From these inequalities the proposition immediately follows.

Proposition 3.16. If $\phi \in \mathfrak{A}=\mathfrak{A}\left(B_{0}, B_{x}, B_{*}, \Sigma_{0}\left(x_{\infty}, \delta\right), \varepsilon\right)$, then we have $\mathrm{e}^{-x} x^{-\sigma} \mathcal{I}\left[\mathrm{e}^{x} x^{\sigma-1} \phi\right]$, $\mathrm{e}^{x} x^{\sigma} \mathcal{I}\left[\mathrm{e}^{-x} x^{-\sigma-1} \phi\right] \in \mathfrak{A}$, and $\left\|\mathrm{e}^{-x} x^{-\sigma} \mathcal{I}\left[\mathrm{e}^{x} x^{\sigma-1} \phi\right]\right\|,\left\|\mathrm{e}^{x} x^{\sigma} \mathcal{I}\left[\mathrm{e}^{-x} x^{-\sigma-1} \phi\right]\right\| \leq(1+2 / \sin \delta)\|\phi\|$.

Proof. For $\phi=\sum_{n=1}^{\infty} p_{n}^{+}(x)\left(\mathrm{e}^{x} x^{\sigma-1}\right)^{n}+\sum_{n=1}^{\infty} p_{n}^{-}(x)\left(\mathrm{e}^{-x} x^{-\sigma-1}\right)^{n}+p_{0}(x) x^{-1} \in \mathfrak{A}$, we have

$$
\mathrm{e}^{x} x^{\sigma-1} \phi=\sum_{n=1}^{\infty} \tilde{p}_{n}^{+}(x)\left(\mathrm{e}^{x} x^{\sigma-1}\right)^{n}+\sum_{n=1}^{\infty} \tilde{p}_{n}^{-}(x)\left(\mathrm{e}^{-x} x^{-\sigma-1}\right)^{n}+\tilde{p}_{0}(x) x^{-1}
$$


with

$$
\begin{aligned}
& \tilde{p}_{n}^{+}(x)=p_{n-1}^{+}(x) \quad \text { for } n \geq 2, \quad \tilde{p}_{1}^{+}(x)=x^{-1} p_{0}(x), \\
& \tilde{p}_{0}(x)=x^{-1} p_{1}^{-}(x), \quad \tilde{p}_{n}^{-}(x)=x^{-2} p_{n+1}^{-}(x) \quad \text { for } n \geq 1 .
\end{aligned}
$$

By the definition of the primitive function

$$
\mathcal{I}\left[\mathrm{e}^{x} x^{\sigma-1} \phi\right]=\sum_{n=1}^{\infty} \tilde{P}_{n}^{+}(x)\left(\mathrm{e}^{x} x^{\sigma-1}\right)^{n}+\sum_{n=1}^{\infty} \tilde{P}_{n}^{-}(x)\left(\mathrm{e}^{-x} x^{-\sigma-1}\right)^{n}+\tilde{P}_{0}(x) x^{-1} \in \hat{\mathfrak{A}}
$$

with $\tilde{P}_{n}^{ \pm}(x)=I_{\gamma(x)}^{ \pm n}\left(\tilde{p}_{n}^{ \pm}(x)\right), \tilde{P}_{0}(x)=I_{\gamma(x)}^{0}\left(\tilde{p}_{0}(x)\right)$, and then

$$
\mathrm{e}^{-x} x^{-\sigma} \mathcal{I}\left[\mathrm{e}^{x} x^{\sigma-1} \phi\right]=\sum_{n=1}^{\infty} P_{n}^{*+}(x)\left(\mathrm{e}^{x} x^{\sigma-1}\right)^{n}+\sum_{n=1}^{\infty} P_{n}^{*-}(x)\left(\mathrm{e}^{-x} x^{-\sigma-1}\right)^{n}+P_{0}^{*}(x) x^{-1},
$$

where

$$
\begin{aligned}
& P_{n}^{*+}(x)=x^{-1} \tilde{P}_{n+1}^{+}(x) \quad \text { for } n \geq 1, \quad P_{0}^{*}(x)=x^{-1} \tilde{P}_{1}^{+}(x), \\
& P_{1}^{*-}(x)=\tilde{P}_{0}(x), \quad P_{n}^{*-}(x)=x \tilde{P}_{n-1}^{-}(x)=O\left(x^{-1}\right) \quad \text { for } n \geq 2 .
\end{aligned}
$$

This implies $\mathrm{e}^{-x} x^{-\sigma} \mathcal{I}\left[\mathrm{e}^{x} x^{\sigma-1} \phi\right] \in \hat{\mathfrak{A}}$. Since, for $n \geq 1$,

$$
\begin{aligned}
P_{n}^{*+}(x) & =x^{-1} \tilde{P}_{n+1}^{+}(x)=-x^{-1} \int_{\gamma(x)}\left(\mathrm{e}^{x} x^{\sigma-1}\right)^{-n-1}\left(\mathrm{e}^{\xi} \xi^{\sigma-1}\right)^{n+1} \tilde{p}_{n+1}^{+}(\xi) \mathrm{d} \xi \\
& =-\int_{\gamma(x)}\left(\mathrm{e}^{x} x^{\sigma}\right)^{-n-1} x^{n}\left(\mathrm{e}^{\xi} \xi^{\sigma}\right)^{n+1} \xi^{-n} p_{n}^{+}(\xi) \frac{\mathrm{d} \xi}{\xi}
\end{aligned}
$$

we have $\left|P_{n}^{*+}(\tilde{x})\right| \leq(1+2 / \sin \delta) M\left(p_{n}^{+},|\tilde{x}|\right) \leq(1+2 / \sin \delta) M\left(p_{n}^{+},|x|\right)$ for any $x, \tilde{x} \in \Sigma_{0}\left(x_{\infty}, \delta\right)$ such that $|\tilde{x}| \geq|x|$, which implies $M\left(P_{n}^{*+},|x|\right) \leq(1+2 / \sin \delta) M\left(p_{n}^{+},|x|\right)$. Similarly, we can verify $M\left(P_{0}^{*},|x|\right) \leq(1+2 / \sin \delta) M\left(p_{0},|x|\right), M\left(P_{n}^{*-},|x|\right) \leq(1+2 / \sin \delta) M\left(p_{n}^{-},|x|\right)$. From these estimates the proposition immediately follows.

\subsection{Families $\mathfrak{A}_{+}, \mathfrak{A}_{-}$}

In addition to $B_{0}$, let $\tilde{B} \subset \mathbb{C}$ be a given domain, and let $\sigma_{0}=-2 \theta_{x}-\theta_{\infty}$. For given numbers $\Theta_{1}$ and $\Theta_{2}$ such that $\pi / 2<\Theta_{1}<\Theta_{2}<3 \pi / 2$, denote by $\Sigma_{\pi}\left(\Theta_{1}, \Theta_{2} ; x_{\infty}\right)$ the sector defined by $\Theta_{1}<\arg x<\Theta_{2},|x|>x_{\infty}$.

Let $\hat{\mathfrak{A}}_{+}=\hat{\mathfrak{A}}_{+}\left(B_{0}, \tilde{B}, \Sigma_{\pi}\left(\Theta_{1}, \Theta_{2} ; x_{\infty}\right)\right)$ be the family of formal series of the form

$$
\phi=\phi(\mathbf{c}, x)=\sum_{n=1}^{\infty} p_{n}^{+}(x)\left(\mathrm{e}^{x} x^{\sigma_{0}}\right)^{n}+p_{0}(x) x^{-1},
$$

strictly the family of pairs $\left(\phi,\left\{p_{n}^{+}(x), p_{0}(x)\right\}_{n \in \mathbb{N}}\right)$, where $p_{n}^{+}(x)$ and $p_{0}(x)$ are holomorphic in $(\mathbf{c}, x) \in B_{0} \times \tilde{B} \times \Sigma_{\pi}\left(\Theta_{1}, \Theta_{2} ; x_{\infty}\right)$ and admit asymptotic representations

$$
p_{n}^{+}(x) \sim \sum_{m=0}^{\infty} p_{n m}^{+} x^{-m}, \quad p_{0}(x) \sim \sum_{m=0}^{\infty} p_{0 m} x^{-m}
$$

with $p_{0 m}, p_{n m}^{+} \in \mathbb{Q}\left[\theta_{0}, \theta_{x}, \theta_{\infty}, c_{0}, c_{0}^{-1}, c_{x}\right] \subset \mathbb{Q}_{*}$ uniformly in $\mathbf{c} \in B_{0} \times \tilde{B}$ as $x \rightarrow \infty$ through $\Sigma_{\pi}\left(\Theta_{1}, \Theta_{2} ; x_{\infty}\right)$. Furthermore, for $\phi$ above set

$$
\|\phi\|_{+}=\|\phi\|_{+}(x, \eta)=\|\phi\|_{+_{\mathbf{c}}}(x, \eta)=\sum_{n=1}^{\infty} M_{+}\left(p_{n}^{+},|x|\right)|\eta|^{n}+M_{+}\left(p_{0},|x|\right)|x|^{-1}
$$


with

$$
M_{+}(p,|x|)=M_{+\mathbf{c}}(p,|x|)=\sup \left\{|p(\mathbf{c}, \xi)| ;|\xi| \geq|x|, \xi \in \Sigma_{\pi}\left(\Theta_{1}, \Theta_{2} ; x_{\infty}\right)\right\} .
$$

Let $\mathfrak{A}_{+}=\mathfrak{A}_{+}\left(B_{0}, \tilde{B}, \Sigma_{\pi}\left(\Theta_{1}, \Theta_{2} ; x_{\infty}\right), \varepsilon\right)\left(\subset \hat{\mathfrak{A}}_{+}\right)$be the family of series $\phi \in \hat{\mathfrak{A}}_{+}$such that $\|\phi\|_{+_{\mathbf{c}}}(x, \eta)$ converges uniformly in $(\mathbf{c}, x, \eta) \in B_{0} \times \tilde{B} \times \Xi_{+}\left(\Sigma_{\pi}\left(\Theta_{1}, \Theta_{2} ; x_{\infty}\right), \varepsilon\right)$, where

$$
\Xi_{+}\left(\Sigma_{\pi}\left(\Theta_{1}, \Theta_{2} ; x_{\infty}\right), \varepsilon\right)=\bigcup_{x \in \Sigma_{\pi}\left(\Theta_{1}, \Theta_{2} ; x_{\infty}\right)}\{x\} \times\{\eta ;|\eta|<\varepsilon\} .
$$

Then every $\phi \in \mathfrak{A}_{+}\left(B_{0}, \tilde{B}, \Sigma_{\pi}\left(\Theta_{1}, \Theta_{2} ; x_{\infty}\right), \varepsilon\right)$ is holomorphic in $(\mathbf{c}, x)$ in the domain $B_{0} \times \tilde{B} \times$ $\left(\Sigma_{\pi}\left(\Theta_{1}, \Theta_{2} ; x_{\infty}\right) \cap\left\{x ;\left|\mathrm{e}^{x} x^{\sigma_{0}}\right|<\varepsilon\right\}\right)$, and satisfies $|\phi(\mathbf{c}, x)| \leq\|\phi\|_{+_{\mathbf{c}}}\left(x, \mathrm{e}^{x} x^{\sigma_{0}}\right)$; and we may similarly verify properties corresponding to those in Propositions 3.1 and 3.3. The primitive function of $\phi \in \hat{\mathfrak{A}}_{+}$or $\mathfrak{A}_{+}$is also defined by replacing $\left(\gamma(x), \mathrm{e}^{x} x^{\sigma-1}\right)$ by $\left(\gamma_{\pi}(x), \mathrm{e}^{x} x^{\sigma_{0}}\right)$ (cf. Lemma 3.9). Then we obtain the same conclusions as in Propositions 3.14 and 3.15. Note that the constant $1+2 / \sin \delta$ in Proposition 3.15 may be replaced by $2 / \min \left\{\left|\cos \Theta_{1}\right|,\left|\cos \Theta_{2}\right|\right\}$. Instead of Proposition 3.16 we have

Proposition 3.17. If $\phi \in \mathfrak{A}_{+}=\mathfrak{A}_{+}\left(B_{0}, \tilde{B}, \Sigma_{\pi}\left(\Theta_{1}, \Theta_{2} ; x_{\infty}\right)\right.$, $\left.\varepsilon\right)$, then $\left(\mathrm{e}^{x} x^{\sigma_{0}}\right)^{-n} \mathcal{I}\left[\left(\mathrm{e}^{x} x^{\sigma_{0}}\right)^{n} \phi\right] \in$ $\mathfrak{A}_{+}$and $\left\|\left(\mathrm{e}^{x} x^{\sigma_{0}}\right)^{-n} \mathcal{I}\left[\left(\mathrm{e}^{x} x^{\sigma_{0}}\right)^{n} \phi\right]\right\| \leq 2\|\phi\| / \min \left\{\left|\cos \Theta_{1}\right|,\left|\cos \Theta_{2}\right|\right\}$ for every $n \geq 1$.

Remark 3.18. If $\phi \in \mathfrak{A}\left(B_{0}, B_{x}, B_{*}, \Sigma_{0}\left(x_{\infty}, \delta\right), \varepsilon\right) \cap \hat{\mathfrak{A}}_{+}\left(B_{0}, \tilde{B}, \Sigma_{\pi}\left(\pi / 2+\delta, \pi-\delta ; x_{\infty}\right), \varepsilon\right)$, then $\phi \in \mathfrak{A}_{+}\left(B_{0}, \tilde{B}, \Sigma_{\pi}\left(\pi / 2+\delta, \pi-\delta ; x_{\infty}\right), \varepsilon\right)$, and $\|\phi\|_{+} \leq\|\phi\|$.

In the sector $\Sigma_{0}\left(\Theta_{1}^{\prime}, \Theta_{2}^{\prime}, x_{\infty}\right):-\pi / 2<\Theta_{1}^{\prime}<\arg x<\Theta_{2}^{\prime}<\pi / 2$, we may similarly define the family $\hat{\mathfrak{A}}_{-}=\hat{\mathfrak{A}}_{-}\left(\tilde{B}, B_{x}, \Sigma_{0}\left(\Theta_{1}^{\prime}, \Theta_{2}^{\prime} ; x_{\infty}\right)\right)$ and $\mathfrak{A}_{-}=\mathfrak{A}_{-}\left(\tilde{B}, B_{x}, \Sigma_{0}\left(\Theta_{1}^{\prime}, \Theta_{2}^{\prime} ; x_{\infty}\right), \varepsilon\right)$ with $\sigma_{0}^{\prime}=$ $2 \theta_{0}+\theta_{\infty}$, which have similar properties.

\section{Equation (1.3) and a system of integral equations}

We would like to construct a general solution of (1.3) under the restrictions (a) and (b). A generic form of a pair of matrices $\Lambda_{0}, \Lambda_{x}$ satisfying $\left(\Lambda_{0}+\Lambda_{x}\right)_{11}=-\left(\Lambda_{0}+\Lambda_{x}\right)_{22}=-\theta_{\infty} / 2$ and having the eigenvalues $\pm \theta_{0} / 2, \pm \theta_{x} / 2$, respectively, may be given by

$$
\Lambda_{0}=\frac{1}{4}\left(\sigma-\theta_{\infty}\right) J+\gamma_{+}^{0} \Delta_{+}+\gamma_{-}^{0} \Delta_{-}, \quad \Lambda_{x}=-\frac{1}{4}\left(\sigma+\theta_{\infty}\right) J+\gamma_{+}^{x} \Delta_{+}+\gamma_{-}^{x} \Delta_{-}
$$

with

$$
\gamma_{ \pm}^{0}= \pm \frac{c_{0}^{ \pm 1}}{4}\left(\sigma \pm 2 \theta_{0}-\theta_{\infty}\right), \quad \gamma_{ \pm}^{x}=\mp \frac{c_{x}^{ \pm 1}}{4}\left(\sigma \mp 2 \theta_{x}+\theta_{\infty}\right)
$$

(cf. Theorem 2.1), where $\sigma, c_{0}, c_{x}$ are arbitrary. For a solution $\left(A_{0}(x), A_{x}(x)\right)$ of $(1.3)$, let us set

$$
\begin{aligned}
& A_{0}(x)=x^{-(1 / 4)\left(\sigma+\theta_{\infty}\right) J}\left(\Lambda_{0}+\Phi_{0}(x)\right) x^{(1 / 4)\left(\sigma+\theta_{\infty}\right) J} \\
& A_{x}(x)=\mathrm{e}^{(x / 2) J} x^{(1 / 4)\left(\sigma-\theta_{\infty}\right) J}\left(\Lambda_{x}+\Phi_{x}(x)\right) x^{-(1 / 4)\left(\sigma-\theta_{\infty}\right) J} \mathrm{e}^{-(x / 2) J}
\end{aligned}
$$

(cf. (4.7) and (4.8)). If $\Phi_{0}(x), \Phi_{x}(x) \rightarrow 0$ along some curve tending to $\infty$, then $\left(A_{0}(x), A_{x}(x)\right)$ satisfies (a) and (b), and (1.1) has the isomonodromy property. In checking (a) and (b) we use $(\mathrm{d} / \mathrm{d} x) \operatorname{tr} A_{0}(x)=(\mathrm{d} / \mathrm{d} x) \operatorname{tr} A_{x}(x) \equiv 0,(\mathrm{~d} / \mathrm{d} x) \operatorname{det} A_{0}(x)=(\mathrm{d} / \mathrm{d} x) \operatorname{det} A_{x}(x) \equiv 0$. Indeed, if $\operatorname{det} A_{0}(x) \not \equiv 0$, then $(\mathrm{d} / \mathrm{d} x) A_{0}(x)=x^{-1}\left(A_{x}(x)-A_{0}(x) A_{x}(x) A_{0}(x)^{-1}\right) A_{0}(x)$, and hence $(\mathrm{d} / \mathrm{d} x) \operatorname{det} A_{0}(x)=x^{-1} \operatorname{tr}\left(A_{x}(x)-A_{0}(x) A_{x}(x) A_{0}(x)^{-1}\right) \operatorname{det} A_{0}(x) \equiv 0$. 
In what follows we change (1.3) into a suitable nonlinear system, and construct a solution of it as mentioned above. Let

$$
A_{0}=f_{0} J+f_{+} \Delta_{+}+f_{-} \Delta_{-}, \quad A_{x}=g_{0} J+g_{+} \Delta_{+}+g_{-} \Delta_{-}
$$

with $g_{0}=-f_{0}-\theta_{\infty} / 2$. Then, (1.3) is equivalent to a system of nonlinear equations

$$
\begin{array}{ll}
x f_{0}^{\prime}=f_{-} g_{+}-f_{+} g_{-}, & g_{0}=-f_{0}-\theta_{\infty} / 2, \\
x f_{+}^{\prime}=2\left(f_{+} g_{0}-f_{0} g_{+}\right), & x g_{+}^{\prime}=2\left(f_{0} g_{+}-f_{+} g_{0}\right)+x g_{+}, \\
x f_{-}^{\prime}=2\left(f_{0} g_{-}-f_{-} g_{0}\right), & x g_{-}^{\prime}=2\left(f_{-} g_{0}-f_{0} g_{-}\right)-x g_{-} .
\end{array}
$$

We set

$$
f_{0}=\left(\sigma-\theta_{\infty}\right) / 4+\varphi, \quad g_{0}=-\left(\sigma+\theta_{\infty}\right) / 4-\varphi
$$

to write (4.3) in the form

$$
\begin{aligned}
& x \varphi^{\prime}=f_{-} g_{+}-f_{+} g_{-}, \\
& x f_{+}^{\prime}=-(1 / 2)\left(\left(\sigma+\theta_{\infty}\right) f_{+}+\left(\sigma-\theta_{\infty}\right) g_{+}\right)-2\left(\varphi f_{+}+\varphi g_{+}\right), \\
& x f_{-}^{\prime}=(1 / 2)\left(\left(\sigma+\theta_{\infty}\right) f_{-}+\left(\sigma-\theta_{\infty}\right) g_{-}\right)+2\left(\varphi f_{-}+\varphi g_{-}\right), \\
& x g_{+}^{\prime}=x g_{+}+(1 / 2)\left(\left(\sigma-\theta_{\infty}\right) g_{+}+\left(\sigma+\theta_{\infty}\right) f_{+}\right)+2\left(\varphi g_{+}+\varphi f_{+}\right), \\
& x g_{-}^{\prime}=-x g_{-}-(1 / 2)\left(\left(\sigma-\theta_{\infty}\right) g_{-}+\left(\sigma+\theta_{\infty}\right) f_{-}\right)-2\left(\varphi g_{-}+\varphi f_{-}\right) .
\end{aligned}
$$

The following fact may be verified by an argument analogous to that of [25, Section 10] (see also [27, Chapter 4]).

Lemma 4.1. By the change of variables $\mathbf{y}=\left(I+p(x) \Delta_{+}\right) \mathbf{z}$ the linear system

$$
x \frac{\mathrm{d} \mathbf{y}}{\mathrm{d} x}=\left(\left(\begin{array}{ll}
0 & 0 \\
0 & 1
\end{array}\right) x+\frac{1}{2}\left(\begin{array}{cc}
-\left(\sigma+\theta_{\infty}\right) & -\left(\sigma-\theta_{\infty}\right) \\
\sigma+\theta_{\infty} & \sigma-\theta_{\infty}
\end{array}\right)\right) \mathbf{y}
$$

is taken to

$$
\left.x \frac{\mathrm{d} \mathbf{z}}{\mathrm{d} x}=\left(\begin{array}{ll}
0 & 0 \\
0 & 1
\end{array}\right) x+\frac{1}{2}\left(\begin{array}{cc}
-\left(\sigma+\theta_{\infty}\right)(1+p(x)) & 0 \\
\sigma+\theta_{\infty} & \sigma-\theta_{\infty}+\left(\sigma+\theta_{\infty}\right) p(x)
\end{array}\right)\right) \mathbf{z},
$$

and by $\mathbf{z}=\left(I+q(x) \Delta_{-}\right) \mathbf{w}$ the last system is reduced to

$$
\left.x \frac{\mathrm{d} \mathbf{w}}{\mathrm{d} x}=\left(\begin{array}{ll}
0 & 0 \\
0 & 1
\end{array}\right) x+\frac{1}{2}\left(\begin{array}{cc}
-\left(\sigma+\theta_{\infty}\right)(1+p(x)) & 0 \\
0 & \sigma-\theta_{\infty}+\left(\sigma+\theta_{\infty}\right) p(x)
\end{array}\right)\right) \mathbf{w} .
$$

Here $p(x)$ and $q(x)$ satisfy

$$
\begin{aligned}
& x p^{\prime}(x)+x p(x)+(1 / 2)(1+p(x))\left(\sigma-\theta_{\infty}+\left(\sigma+\theta_{\infty}\right) p(x)\right)=0, \\
& x q^{\prime}(x)-x q(x)-(1 / 2)\left(\left(\sigma+\theta_{\infty}\right)(1+(1+2 p(x)) q(x))+\left(\sigma-\theta_{\infty}\right) q(x)\right)=0,
\end{aligned}
$$

and admit the asymptotic representations

$$
\begin{array}{ll}
p(x)=-(1 / 2)\left(\sigma-\theta_{\infty}\right)\left(x^{-1}+(1-\sigma) x^{-2}+\left[x^{-3}\right]\right) & \text { for }|\arg x-\pi|<3 \pi / 2-\delta, \\
q(x)=-(1 / 2)\left(\sigma+\theta_{\infty}\right)\left(x^{-1}-(1+\sigma) x^{-2}+\left[x^{-3}\right]\right) & \text { for }|\arg x-\pi / 2|<\pi-\delta,
\end{array}
$$

whose coefficients are in $\mathbb{Q}\left[\theta_{\infty}, \sigma\right]$. 


\section{Remark 4.2.}

(1) In Lemma 4.1, $p(x)$ may be replaced by $\tilde{p}(x)$ having the same asymptotic representation in the sector $|\arg x+\pi|<3 \pi / 2-\delta$. The diagonalisation is possible by $(\tilde{p}(x), q(x))$ for $|\arg x+\pi / 2|<\pi-\delta$ as well as $(p(x), q(x))$ for $|\arg x-\pi / 2|<\pi-\delta$.

(2) By the substitution $\left(\sigma-\theta_{\infty}, \sigma+\theta_{\infty}, x\right) \mapsto\left(-\left(\sigma-\theta_{\infty}\right),-\left(\sigma+\theta_{\infty}\right), \mathrm{e}^{\pi \mathrm{i}} x\right)$ or $\mapsto(-(\sigma-$ $\left.\left.\theta_{\infty}\right),-\left(\sigma+\theta_{\infty}\right), \mathrm{e}^{-\pi \mathrm{i}} x\right)$, we obtain

$$
\begin{aligned}
& p^{*}(x)=-(1 / 2)\left(\sigma-\theta_{\infty}\right)\left(x^{-1}-(1+\sigma) x^{-2}+\left[x^{-3}\right]\right), \\
& q^{*}(x)=-(1 / 2)\left(\sigma+\theta_{\infty}\right)\left(x^{-1}+(1-\sigma) x^{-2}+\left[x^{-3}\right]\right)
\end{aligned}
$$

such that

$$
x \frac{\mathrm{d} \mathbf{y}}{\mathrm{d} x}=\left(\left(\begin{array}{cc}
0 & 0 \\
0 & -1
\end{array}\right) x+\frac{1}{2}\left(\begin{array}{cc}
\sigma+\theta_{\infty} & \sigma-\theta_{\infty} \\
-\left(\sigma+\theta_{\infty}\right) & -\left(\sigma-\theta_{\infty}\right)
\end{array}\right)\right) \mathbf{y}
$$

is changed into

$$
\left.x \frac{\mathrm{d} \mathbf{w}}{\mathrm{d} x}=\left(\begin{array}{cc}
0 & 0 \\
0 & -1
\end{array}\right) x+\frac{1}{2}\left(\begin{array}{cc}
\left(\sigma+\theta_{\infty}\right)\left(1+p^{*}(x)\right) & 0 \\
0 & -\left(\sigma-\theta_{\infty}\right)-\left(\sigma+\theta_{\infty}\right) p^{*}(x)
\end{array}\right)\right) \mathbf{w} .
$$

Remark 4.3. The proof of Lemma 4.1 depends on the following fact: from every point in the sector $|\arg x-\pi|<3 \pi / 2-\delta$ (respectively, $|\arg x|<3 \pi / 2-\delta$ ) one may draw a line such that it is contained in the sector and that $\operatorname{Re} x \rightarrow-\infty$ (respectively, $\rightarrow \infty$ ) along it.

By the facts above the linear parts of (4.4) may be diagonalised, that is, there exists a transformation of the form

$$
\begin{aligned}
& f_{+}=(1+p q) u_{+}+p v_{+}=\left(1+\left[x^{-2}\right]\right) u_{+}-x^{-1}\left(\left(\sigma-\theta_{\infty}\right) / 2+\left[x^{-1}\right]\right) v_{+}, \\
& g_{+}=q u_{+}+v_{+}=x^{-1}\left(-\left(\sigma+\theta_{\infty}\right) / 2+\left[x^{-1}\right]\right) u_{+}+v_{+}, \\
& f_{-}=\left(1+p^{*} q^{*}\right) u_{-}+p^{*} v_{-}=\left(1+\left[x^{-2}\right]\right) u_{-}-x^{-1}\left(\left(\sigma-\theta_{\infty}\right) / 2+\left[x^{-1}\right]\right) v_{-}, \\
& g_{-}=q^{*} u_{-}+v_{-}=x^{-1}\left(-\left(\sigma+\theta_{\infty}\right) / 2+\left[x^{-1}\right]\right) u_{-}+v_{-}
\end{aligned}
$$

that takes (4.4) to

$$
\begin{aligned}
x \varphi^{\prime}= & \left(1+\left[x^{-2}\right]\right) u_{-} v_{+}-\left(1+\left[x^{-2}\right]\right) u_{+} v_{-} \\
& +\left(\sigma+\theta_{\infty}+\left[x^{-1}\right]\right) x^{-2} u_{+} u_{-}+\left(\sigma-\theta_{\infty}+\left[x^{-1}\right]\right) x^{-2} v_{+} v_{-}, \\
x u_{+}^{\prime}= & \left(-\left(\sigma+\theta_{\infty}\right) / 2+\left[x^{-1}\right]\right) u_{+}-2 \varphi\left(\left(1+\left[x^{-1}\right]\right) u_{+}+\left(1+\left[x^{-1}\right]\right) v_{+}\right), \\
x v_{+}^{\prime}= & x v_{+}+\left(\left(\sigma-\theta_{\infty}\right) / 2+\left[x^{-1}\right]\right) v_{+}+2 \varphi\left(\left(1+\left[x^{-1}\right]\right) u_{+}+\left(1+\left[x^{-1}\right]\right) v_{+}\right), \\
x u_{-}^{\prime}= & \left(\left(\sigma+\theta_{\infty}\right) / 2+\left[x^{-1}\right]\right) u_{-}+2 \varphi\left(\left(1+\left[x^{-1}\right]\right) u_{-}+\left(1+\left[x^{-1}\right]\right) v_{-}\right), \\
x v_{-}^{\prime}= & -x v_{-}\left(\left(\sigma-\theta_{\infty}\right) / 2+\left[x^{-1}\right]\right) v_{-}-2 \varphi\left(\left(1+\left[x^{-1}\right]\right) u_{-}+\left(1+\left[x^{-1}\right]\right) v_{-}\right),
\end{aligned}
$$

where $\left[x^{-1}\right],\left[x^{-2}\right], \ldots$ are valid in the sector $|\arg x-\pi / 2|<\pi-\delta$. Rewriting, e.g., the last equation in the form

$$
\left(\mathrm{e}^{x} x^{\left(\sigma-\theta_{\infty}\right) / 2}\left(1+\left[x^{-1}\right]\right) v_{-}\right)^{\prime}=-2 \mathrm{e}^{x} x^{\left(\sigma-\theta_{\infty}\right) / 2-1} \varphi\left(\left(1+\left[x^{-1}\right]\right) u_{-}+\left(1+\left[x^{-1}\right]\right) v_{-}\right),
$$

and setting

$$
\begin{aligned}
& x^{\left(\sigma+\theta_{\infty}\right) / 2}\left(1+\left[x^{-1}\right]\right) u_{+}=\gamma_{+}^{0}+\varphi_{+}, \quad \mathrm{e}^{-x} x^{-\left(\sigma-\theta_{\infty}\right) / 2}\left(1+\left[x^{-1}\right]\right) v_{+}=\gamma_{+}^{x}+\psi_{+}, \\
& x^{-\left(\sigma+\theta_{\infty}\right) / 2}\left(1+\left[x^{-1}\right]\right) u_{-}=\gamma_{-}^{0}+\varphi_{-}, \quad \mathrm{e}^{x} x^{\left(\sigma-\theta_{\infty}\right) / 2}\left(1+\left[x^{-1}\right]\right) v_{-}=\gamma_{-}^{x}+\psi_{-}
\end{aligned}
$$


with $\gamma_{ \pm}^{0}$ and $\gamma_{ \pm}^{x}$ as in (4.1), we arrive at a system of integral equations of the form

$$
\begin{aligned}
\varphi= & \mathcal{I}\left[\mathrm{e}^{x} x^{\sigma-1}(1)_{x}\left(\gamma_{-}^{0}+\varphi_{-}\right)\left(\gamma_{+}^{x}+\psi_{+}\right)-\mathrm{e}^{-x} x^{-\sigma-1}(1)_{x}\left(\gamma_{+}^{0}+\varphi_{+}\right)\left(\gamma_{-}^{x}+\psi_{-}\right)\right] \\
& +\mathcal{I}\left[\left(\sigma+\theta_{\infty}+\left[x^{-1}\right]\right) x^{-3}\left(\gamma_{+}^{0}+\varphi_{+}\right)\left(\gamma_{-}^{0}+\varphi_{-}\right)\right. \\
& \left.+\left(\sigma-\theta_{\infty}+\left[x^{-1}\right]\right) x^{-3}\left(\gamma_{+}^{x}+\psi_{+}\right)\left(\gamma_{-}^{x}+\psi_{-}\right)\right], \\
\varphi_{+} & =-2 \mathcal{I}\left[\varphi\left(x^{-1}(1)_{x}\left(\gamma_{+}^{0}+\varphi_{+}\right)+\mathrm{e}^{x} x^{\sigma-1}(1)_{x}\left(\gamma_{+}^{x}+\psi_{+}\right)\right)\right], \\
\psi_{+} & =2 \mathcal{I}\left[\varphi\left(x^{-1}(1)_{x}\left(\gamma_{+}^{x}+\psi_{+}\right)+\mathrm{e}^{-x} x^{-\sigma-1}(1)_{x}\left(\gamma_{+}^{0}+\varphi_{+}\right)\right)\right], \\
\varphi_{-} & =2 \mathcal{I}\left[\varphi\left(x^{-1}(1)_{x}\left(\gamma_{-}^{0}+\varphi_{-}\right)+\mathrm{e}^{-x} x^{-\sigma-1}(1)_{x}\left(\gamma_{-}^{x}+\psi_{-}\right)\right)\right], \\
\psi_{-} & =-2 \mathcal{I}\left[\varphi\left(x^{-1}(1)_{x}\left(\gamma_{-}^{x}+\psi_{-}\right)+\mathrm{e}^{x} x^{\sigma-1}(1)_{x}\left(\gamma_{-}^{0}+\varphi_{-}\right)\right)\right] .
\end{aligned}
$$

Here $(1)_{x}$ denotes $1+\left[x^{-1}\right]$, and the functions $\varphi, \varphi_{ \pm}, \psi_{ \pm}$and the products $\varphi_{-} \psi_{+}, \varphi_{+} \psi_{-}$, $\ldots$ are supposed to be at least in $\hat{\mathfrak{A}}$. If we succeed in constructing $\varphi, \varphi_{ \pm}, \psi_{ \pm} \in \mathfrak{A}$, then, by Propositions 3.14 through $3.16,\left(A_{0}(x), A_{x}(x)\right)$ with

$$
\begin{aligned}
& f_{0}=\left(\sigma-\theta_{\infty}\right) / 4+\varphi, \quad g_{0}=-\left(\sigma+\theta_{\infty}\right) / 4-\varphi \\
& f_{+}=x^{-\left(\sigma+\theta_{\infty}\right) / 2}\left((1)_{x}\left(\gamma_{+}^{0}+\varphi_{+}\right)-\left(\left(\sigma-\theta_{\infty}\right) / 2+\left[x^{-1}\right]\right) \mathrm{e}^{x} x^{\sigma-1}\left(\gamma_{+}^{x}+\psi_{+}\right)\right), \\
& g_{+}=\mathrm{e}^{x} x^{\left(\sigma-\theta_{\infty}\right) / 2}\left((1)_{x}\left(\gamma_{+}^{x}+\psi_{+}\right)-\left(\left(\sigma+\theta_{\infty}\right) / 2+\left[x^{-1}\right]\right) \mathrm{e}^{-x} x^{-\sigma-1}\left(\gamma_{+}^{0}+\varphi_{+}\right)\right), \\
& f_{-}=x^{\left(\sigma+\theta_{\infty}\right) / 2}\left((1)_{x}\left(\gamma_{-}^{0}+\varphi_{-}\right)-\left(\left(\sigma-\theta_{\infty}\right) / 2+\left[x^{-1}\right]\right) \mathrm{e}^{-x} x^{-\sigma-1}\left(\gamma_{-}^{x}+\psi_{-}\right)\right), \\
& g_{-}=\mathrm{e}^{-x} x^{-\left(\sigma-\theta_{\infty}\right) / 2}\left((1)_{x}\left(\gamma_{-}^{x}+\psi_{-}\right)-\left(\left(\sigma+\theta_{\infty}\right) / 2+\left[x^{-1}\right]\right) \mathrm{e}^{x} x^{\sigma-1}\left(\gamma_{-}^{0}+\varphi_{-}\right)\right)
\end{aligned}
$$

is a solution of (1.3) written as (4.2). Then, $\Phi_{0}(x)$ and $\Phi_{x}(x)$ in (4.2) are given by

$$
\begin{aligned}
& \Phi_{0}(x)_{11}=-\Phi_{0}(x)_{22}=\varphi \\
& \Phi_{0}(x)_{12}=\gamma_{+}^{0}\left[x^{-1}\right]+(1)_{x} \varphi_{+}-\left(\left(\sigma-\theta_{\infty}\right) / 2+\left[x^{-1}\right]\right) \mathrm{e}^{x} x^{\sigma-1}\left(\gamma_{+}^{x}+\psi_{+}\right), \\
& \Phi_{0}(x)_{21}=\gamma_{-}^{0}\left[x^{-1}\right]+(1)_{x} \varphi_{-}-\left(\left(\sigma-\theta_{\infty}\right) / 2+\left[x^{-1}\right]\right) \mathrm{e}^{-x} x^{-\sigma-1}\left(\gamma_{-}^{x}+\psi_{-}\right), \\
& \Phi_{x}(x)_{11}=-\Phi_{x}(x)_{22}=-\varphi \\
& \Phi_{x}(x)_{12}=\gamma_{+}^{x}\left[x^{-1}\right]+(1)_{x} \psi_{+}-\left(\left(\sigma+\theta_{\infty}\right) / 2+\left[x^{-1}\right]\right) \mathrm{e}^{-x} x^{-\sigma-1}\left(\gamma_{+}^{0}+\varphi_{+}\right), \\
& \Phi_{x}(x)_{21}=\gamma_{-}^{x}\left[x^{-1}\right]+(1)_{x} \psi_{-}-\left(\left(\sigma+\theta_{\infty}\right) / 2+\left[x^{-1}\right]\right) \mathrm{e}^{x} x^{\sigma-1}\left(\gamma_{-}^{0}+\varphi_{-}\right) .
\end{aligned}
$$

Moreover, if $\varphi, \varphi_{ \pm}, \psi_{ \pm}$are such that $\Phi_{0}(x), \Phi_{x}(x) \rightarrow 0$ as $x \rightarrow \infty$ along some curve, then $\left(A_{0}(x), A_{x}(x)\right)$ satisfies (a) and (b), and hence it is a desired solution of (1.3).

Remark 4.4. By $p(x)$ and $p^{*}(x)$ in Lemma 4.1 and Remark 4.2, the linear parts of (4.5) are written in the more detailed form

$$
\begin{aligned}
& x u_{+}^{\prime}=\left(-\left(\sigma+\theta_{\infty}\right) / 2+\kappa(x)\right) u_{+}+\cdots, \\
& x v_{+}^{\prime}=x v_{+}+\left(\left(\sigma-\theta_{\infty}\right) / 2-\kappa(x)\right) v_{+}+\cdots, \\
& x u_{-}^{\prime}=\left(\left(\sigma+\theta_{\infty}\right) / 2-\kappa(x)\right) u_{-}+\cdots, \\
& x v_{-}^{\prime}=-x v_{-}+\left(-\left(\sigma-\theta_{\infty}\right) / 2+\kappa(x)\right) v_{-}+\cdots
\end{aligned}
$$

with $\kappa(x)=\left(\sigma^{2}-\theta_{\infty}^{2}\right) x^{-1} / 4+\left[x^{-2}\right]$, in each appearance $\left[x^{-2}\right]$ not necessarily denoting the same function. Then the expressions of $f_{ \pm}, g_{ \pm}$in (4.7) become

$$
\begin{aligned}
& f_{+}=x^{-\left(\sigma+\theta_{\infty}\right) / 2}\left((1-\kappa(x))\left(\gamma_{+}^{0}+\varphi_{+}\right)-\cdots\right), \\
& g_{+}=\mathrm{e}^{x} x^{\left(\sigma-\theta_{\infty}\right) / 2}\left((1+\kappa(x))\left(\gamma_{+}^{x}+\psi_{+}\right)-\cdots\right), \\
& f_{-}=x^{\left(\sigma+\theta_{\infty}\right) / 2}\left((1+\kappa(x))\left(\gamma_{-}^{0}+\varphi_{-}\right)-\cdots\right), \\
& g_{-}=\mathrm{e}^{-x} x^{-\left(\sigma-\theta_{\infty}\right) / 2}\left((1-\kappa(x))\left(\gamma_{-}^{x}+\psi_{-}\right)-\cdots\right),
\end{aligned}
$$


and the first equation in (4.6) is

$$
\begin{aligned}
\varphi= & \mathcal{I}\left[\mathrm{e}^{x} x^{\sigma-1}(1+2 \kappa(x))\left(\gamma_{-}^{0}+\varphi_{-}\right)\left(\gamma_{+}^{x}+\psi_{+}\right)\right. \\
& \left.-\mathrm{e}^{-x} x^{-\sigma-1}(1-2 \kappa(x))\left(\gamma_{+}^{0}+\varphi_{+}\right)\left(\gamma_{-}^{x}+\psi_{-}\right)\right]+\cdots .
\end{aligned}
$$

\section{Proofs of Theorems 2.1 and 2.8}

\subsection{System of integral equations}

Instead of system (4.6) we deal with

$$
\begin{aligned}
\varphi= & F_{0}\left(x, \varphi, \varphi_{+}, \psi_{+}, \varphi_{-}, \psi_{-}\right):=\mathrm{e}^{x} x^{\sigma-1}(1)_{x}\left(\gamma_{-}^{0}+\varphi_{-}\right)\left(\gamma_{+}^{x}+\psi_{+}\right) \\
& +\mathrm{e}^{-x} x^{-\sigma-1}(1)_{x}\left(\gamma_{+}^{0}+\varphi_{+}\right)\left(\gamma_{-}^{x}+\psi_{-}\right) \\
& -4 \mathcal{I}\left[\mathrm{e}^{x} x^{\sigma-2}(1)_{x} \varphi\left(\gamma_{-}^{0}+\varphi_{-}\right)\left(\gamma_{+}^{x}+\psi_{+}\right)-\mathrm{e}^{-x} x^{-\sigma-2}(1)_{x} \varphi\left(\gamma_{+}^{0}+\varphi_{+}\right)\left(\gamma_{-}^{x}+\psi_{-}\right)\right] \\
& +\mathcal{I}\left[x^{-3}\left(\sigma+\theta_{\infty}+\left[x^{-1}\right]+[1] \varphi\right)\left(\gamma_{+}^{0}+\varphi_{+}\right)\left(\gamma_{-}^{0}+\varphi_{-}\right)\right. \\
& \left.+x^{-3}\left(\sigma-\theta_{\infty}+\left[x^{-1}\right]+[1] \varphi\right)\left(\gamma_{+}^{x}+\psi_{+}\right)\left(\gamma_{-}^{x}+\psi_{-}\right)\right], \\
\varphi_{+} & =F_{+}\left(x, \varphi, \varphi_{+}, \psi_{+}\right):=-2 \mathcal{I}\left[\varphi\left(x^{-1}(1)_{x}\left(\gamma_{+}^{0}+\varphi_{+}\right)+\mathrm{e}^{x} x^{\sigma-1}(1)_{x}\left(\gamma_{+}^{x}+\psi_{+}\right)\right)\right], \\
\psi_{+} & =G_{+}\left(x, \varphi, \varphi_{+}, \psi_{+}\right):=2 \mathcal{I}\left[\varphi\left(x^{-1}(1)_{x}\left(\gamma_{+}^{x}+\psi_{+}\right)+\mathrm{e}^{-x} x^{-\sigma-1}(1)_{x}\left(\gamma_{+}^{0}+\varphi_{+}\right)\right)\right], \\
\varphi_{-} & =F_{-}\left(x, \varphi, \varphi_{-}, \psi_{-}\right):=2 \mathcal{I}\left[\varphi\left(x^{-1}(1)_{x}\left(\gamma_{-}^{0}+\varphi_{-}\right)+\mathrm{e}^{-x} x^{-\sigma-1}(1)_{x}\left(\gamma_{-}^{x}+\psi_{-}\right)\right)\right], \\
\psi_{-} & =G_{-}\left(x, \varphi, \varphi_{-}, \psi_{-}\right):=-2 \mathcal{I}\left[\varphi\left(x^{-1}(1)_{x}\left(\gamma_{-}^{x}+\psi_{-}\right)+\mathrm{e}^{x} x^{\sigma-1}(1)_{x}\left(\gamma_{-}^{0}+\varphi_{-}\right)\right)\right],
\end{aligned}
$$

which is equivalent to (4.6). Indeed, by Proposition 3.14 and integration by parts, we may write the first equation of (4.6) in the form

$$
\varphi=I_{0}+\mathcal{I}\left[\left(\sigma+\theta_{\infty}+\left[x^{-1}\right]\right) x^{-3}\left(\gamma_{+}^{0}+\varphi_{+}\right)\left(\gamma_{-}^{0}+\varphi_{-}\right)+\cdots\right]
$$

with

$$
\begin{aligned}
I_{0}= & \mathcal{I}\left[\mathrm{e}^{x} x^{\sigma-1}(1)_{x}\left(\gamma_{-}^{0}+\varphi_{-}\right)\left(\gamma_{+}^{x}+\psi_{+}\right)-\mathrm{e}^{-x} x^{-\sigma-1}(1)_{x}\left(\gamma_{+}^{0}+\varphi_{+}\right)\left(\gamma_{-}^{x}+\psi_{-}\right)\right] \\
= & \mathrm{e}^{x} x^{\sigma-1}(1)_{x}\left(\gamma_{-}^{0}+\varphi_{-}\right)\left(\gamma_{+}^{x}+\psi_{+}\right)+\mathrm{e}^{-x} x^{-\sigma-1}(1)_{x}\left(\gamma_{+}^{0}+\varphi_{+}\right)\left(\gamma_{-}^{x}+\psi_{-}\right) \\
& -\mathcal{I}\left[\mathrm{e}^{x} x^{\sigma-1}(1)_{x}\left(\psi_{+}^{\prime}\left(\gamma_{-}^{0}+\varphi_{-}\right)+\varphi_{-}^{\prime}\left(\gamma_{+}^{x}+\psi_{+}\right)\right)\right. \\
& \left.+\mathrm{e}^{-x} x^{-\sigma-1}(1)_{x}\left(\psi_{-}^{\prime}\left(\gamma_{+}^{0}+\varphi_{+}\right)+\varphi_{+}^{\prime}\left(\gamma_{-}^{x}+\psi_{-}\right)\right)\right] .
\end{aligned}
$$

The substitution of

$$
\varphi_{+}^{\prime}=-2 \varphi\left(x^{-1}(1)_{x}\left(\gamma_{+}^{0}+\varphi_{+}\right)+\mathrm{e}^{x} x^{\sigma-1}(1)_{x}\left(\gamma_{+}^{x}+\psi_{+}\right)\right),
$$

$\varphi_{-}^{\prime}=\cdots, \psi_{ \pm}^{\prime}=\cdots$ into the last integral yields the first equation of (5.1).

\subsection{Sequences}

To construct a solution of (5.1) we define $\left\{\left(\varphi^{j}, \varphi_{+}^{j}, \psi_{+}^{j}, \varphi_{-}^{j}, \psi_{-}^{j}\right)\right\}_{j \geq 0}$ by

$$
\begin{aligned}
& \varphi^{0}=\varphi_{ \pm}^{0}=\psi_{ \pm}^{0} \equiv 0, \quad \varphi^{j+1}=F_{0}\left(x, \varphi^{j}, \varphi_{+}^{j}, \psi_{+}^{j}, \varphi_{-}^{j}, \psi_{-}^{j}\right), \\
& \varphi_{+}^{j+1}=F_{+}\left(x, \varphi^{j+1}, \varphi_{+}^{j}, \psi_{+}^{j}\right), \quad \psi_{+}^{j+1}=G_{+}\left(x, \varphi^{j+1}, \varphi_{+}^{j}, \psi_{+}^{j}\right), \\
& \varphi_{-}^{j+1}=F_{-}\left(x, \varphi^{j+1}, \varphi_{-}^{j}, \psi_{-}^{j}\right), \quad \psi_{-}^{j+1}=G_{-}\left(x, \varphi^{j+1}, \varphi_{-}^{j}, \psi_{-}^{j}\right)
\end{aligned}
$$

for $j \geq 0$. It is shown by induction on $j$ that $\varphi^{j}, \varphi_{ \pm}^{j}, \psi_{ \pm}^{j}$ are finite sums of $\left(\mathrm{e}^{x} x^{\sigma-1}\right)^{n}[1]$, $\left(\mathrm{e}^{-x} x^{-\sigma-1}\right)^{n}[1]$ and $\left[x^{-1}\right]$, and hence $\varphi^{j}, \varphi_{ \pm}^{j}, \psi_{ \pm}^{j} \in \mathfrak{A}$ for $j \geq 0$. 
Remark 5.1. As long as $|\arg x-\pi / 2|<\pi-\delta$, the path of integration for $\mathcal{I}[\cdot]$ may also be taken to be a line on which $\left|\mathrm{e}^{\xi} \xi^{\sigma-1}\right|$ or $\left|\mathrm{e}^{-\xi} \xi^{-\sigma-1}\right|$ decays exponentially, and hence the asymptotic expansions $\left[x^{-1}\right], \ldots$ in the expressions of $\varphi^{j}, \varphi_{ \pm}^{j}, \psi_{ \pm}^{j}$ are valid in the sector $|\arg x-\pi / 2|<\pi-\delta$ (cf. Example 3.2).

In this section, to simplify the description, for a sequence $\left\{\phi^{j}\right\}$ we write $\triangle \phi^{j}:=\phi^{j}-\phi^{j-1}$.

\subsection{1 $\varphi^{j}, \varphi_{ \pm}^{j}, \psi_{ \pm}^{j}$ for $j=1,2$}

By definition $\varphi^{1}=F_{0}(x, 0,0,0,0,0), \varphi_{ \pm}^{1}=F_{ \pm}\left(x, \varphi^{1}, 0,0\right), \psi_{ \pm}^{1}=G_{ \pm}\left(x, \varphi^{1}, 0,0\right)$, that is

$$
\begin{aligned}
& \varphi^{1}=\gamma_{-}^{0} \gamma_{+}^{x}(1)_{x} \mathrm{e}^{x} x^{\sigma-1}+\gamma_{+}^{0} \gamma_{-}^{x}(1)_{x} \mathrm{e}^{-x} x^{-\sigma-1}+\left(\gamma_{+}^{0} \gamma_{-}^{0}[1]+\gamma_{+}^{x} \gamma_{-}^{x}[1]\right) x^{-2}, \\
& \varphi_{+}^{1}=-2 \gamma_{+}^{0} X_{0}^{1}-\gamma_{+}^{x} X_{+}^{1}, \quad \psi_{+}^{1}=2 \gamma_{+}^{x} X_{0}^{1}+\gamma_{+}^{0} X_{-}^{1} \\
& \varphi_{-}^{1}=2 \gamma_{-}^{0} X_{0}^{1}+\gamma_{-}^{x} X_{-}^{1}, \quad \psi_{-}^{1}=-2 \gamma_{-}^{x} X_{0}^{1}-\gamma_{-}^{0} X_{+}^{1}
\end{aligned}
$$

with

$$
\begin{aligned}
& X_{0}^{1}=\gamma_{-}^{0} \gamma_{+}^{x}(1)_{x} \mathrm{e}^{x} x^{\sigma-2}-\gamma_{+}^{0} \gamma_{-}^{x}(1)_{x} \mathrm{e}^{-x} x^{-\sigma-2}+\left(\gamma_{+}^{0} \gamma_{-}^{0}[1]+\gamma_{+}^{x} \gamma_{-}^{x}[1]\right) x^{-2}, \\
& X_{+}^{1}=\gamma_{-}^{0} \gamma_{+}^{x}(1)_{x} \mathrm{e}^{2 x} x^{2 \sigma-2}-2 \gamma_{+}^{0} \gamma_{-}^{x}(1)_{x} x^{-1}+\left(\gamma_{+}^{0} \gamma_{-}^{0}[1]+\gamma_{+}^{x} \gamma_{-}^{x}[1]\right) \mathrm{e}^{x} x^{\sigma-3}, \\
& X_{-}^{1}=-2 \gamma_{-}^{0} \gamma_{+}^{x}(1)_{x} x^{-1}-\gamma_{+}^{0} \gamma_{-}^{x}(1)_{x} \mathrm{e}^{-2 x} x^{-2 \sigma-2}+\left(\gamma_{+}^{0} \gamma_{-}^{0}[1]+\gamma_{+}^{x} \gamma_{-}^{x}[1]\right) \mathrm{e}^{-x} x^{-\sigma-3},
\end{aligned}
$$

which belong to $\mathfrak{A}=\mathfrak{A}\left(B_{0}, B_{x}, B_{*}, \Sigma_{0}\left(x_{\infty}, \delta\right), \varepsilon\right)$ (cf. Section 3.1). We may choose $x_{\infty}^{1}=$ $x_{\infty}^{1}\left(B_{0}, B_{x}, B_{*}, \delta\right)>x_{\infty}$ depending on $\left(\gamma_{ \pm}^{0}, \gamma_{ \pm}^{x}\right)$ or on $(\mathbf{c}, \sigma) \in B_{0} \times B_{x} \times B_{*}$ in such a way that the following estimates with absolute implied constants are valid for $(\mathbf{c}, \sigma, x) \in B_{0} \times B_{x} \times$ $B_{*} \times \Sigma_{0}\left(x_{\infty}^{1}, \delta\right)$ :

$$
\begin{aligned}
& \left\|\varphi^{1}\right\| \ll\left|\gamma_{-}^{0} \gamma_{+}^{x}\right|\left|\eta x^{-1}\right|+\left|\gamma_{+}^{0} \gamma_{-}^{x}\right|\left|\eta^{-1} x^{-1}\right|+|x|^{-1} \\
& \left\|X_{0}^{1}\right\| \ll|x|^{-1}\left(\left|\gamma_{-}^{0} \gamma_{+}^{x}\right|\left|\eta x^{-1}\right|+\left|\gamma_{+}^{0} \gamma_{-}^{x}\right|\left|\eta^{-1} x^{-1}\right|+1\right) \\
& \left\|X_{+}^{1}\right\| \ll\left|\gamma_{-}^{0} \gamma_{+}^{x}\right|\left|\eta x^{-1}\right|^{2}+\left|\gamma_{+}^{0} \gamma_{-}^{x}\right||x|^{-1}+\left|\eta x^{-1}\right||x|^{-1} \\
& \left\|X_{-}^{1}\right\| \ll\left|\gamma_{-}^{0} \gamma_{+}^{x}\right||x|^{-1}+\left|\gamma_{+}^{0} \gamma_{-}^{x}\right|\left|\eta^{-1} x^{-1}\right|^{2}+\left|\eta^{-1} x^{-1}\right||x|^{-1}
\end{aligned}
$$

Then under the condition that

$$
\left(\gamma_{0}^{*}+\gamma_{1}^{*}+1\right)\left(\gamma_{1}^{*}+1\right) \varepsilon \leq r_{0}<1
$$

with

$$
\gamma_{0}^{*}:=\left|\gamma_{-}^{0} \gamma_{+}^{x}\right|+\left|\gamma_{+}^{0} \gamma_{-}^{x}\right|, \quad \gamma_{1}^{*}:=\left|\gamma_{+}^{0}\right|+\left|\gamma_{-}^{0}\right|+\left|\gamma_{+}^{x}\right|+\left|\gamma_{-}^{x}\right|
$$

for every $(\mathbf{c}, \sigma) \in B_{0} \times B_{x} \times B_{*}$ (cf. Remark 2.3), we have, for $\left|\eta x^{-1}\right|,\left|\eta^{-1} x^{-1}\right|<\varepsilon, x \in \Sigma_{0}\left(x_{\infty}^{1}, \delta\right)$,

$$
\left\|\varphi^{1}\right\| \ll\left(\gamma_{0}^{*}+1\right) \varepsilon, \quad\left\|\varphi_{ \pm}^{1}\right\|,\left\|\psi_{ \pm}^{1}\right\| \ll\left(\gamma_{0}^{*}+1\right)\left(\gamma_{1}^{*}+1\right) \varepsilon,
$$

where $r_{0}$ will be chosen later. By (5.3) we have $\mathrm{e}^{-x} x^{-\sigma} \varphi_{+}^{1} \in \mathfrak{A}$, and, under (5.4), $\left\|\mathrm{e}^{-x} x^{-\sigma} \varphi_{+}^{1}\right\| \ll 1$ for $\left|\eta x^{-1}\right|,\left|\eta^{-1} x^{-1}\right|<\varepsilon, x \in \Sigma_{0}\left(x_{\infty}^{1}, \delta\right)$. Similarly, $\mathrm{e}^{x} x^{\sigma} \varphi_{-}^{1},\left(\mathrm{e}^{x} x^{\sigma}\right)^{ \pm 1} \psi_{ \pm}^{1} \in \mathfrak{A}$, and $\left\|\mathrm{e}^{x} x^{\sigma} \varphi_{-}^{1}\right\|$, $\left\|\left(\mathrm{e}^{x} x^{\sigma}\right)^{ \pm 1} \psi_{ \pm}^{1}\right\| \ll 1$. By $(5.2)$,

$$
\triangle \varphi^{2}=F_{0}\left(x, \varphi^{1}, \varphi_{+}^{1}, \psi_{+}^{1}, \varphi_{-}^{1}, \psi_{-}^{1}\right)-F_{0}(x, 0,0,0,0,0)=X_{0}+I_{1}+I_{2}
$$


with

$$
\begin{aligned}
X_{0}= & \mathrm{e}^{x} x^{\sigma-1}(1)_{x}\left(\gamma_{+}^{x} \varphi_{-}^{1}+\gamma_{-}^{0} \psi_{+}^{1}+\varphi_{-}^{1} \psi_{+}^{1}\right)+\mathrm{e}^{-x} x^{-\sigma-1}(1)_{x}\left(\gamma_{-}^{x} \varphi_{+}^{1}+\gamma_{+}^{0} \psi_{-}^{1}+\varphi_{+}^{1} \psi_{-}^{1}\right), \\
I_{1}= & -4 \mathcal{I}\left[\mathrm{e}^{x} x^{\sigma-2}(1)_{x} \varphi^{1}\left(\gamma_{-}^{0}+\varphi_{-}^{1}\right)\left(\gamma_{+}^{x}+\psi_{+}^{1}\right)-\mathrm{e}^{-x} x^{-\sigma-2}(1)_{x} \varphi^{1}\left(\gamma_{+}^{0}+\varphi_{+}^{1}\right)\left(\gamma_{-}^{x}+\psi_{-}^{1}\right)\right], \\
I_{2}= & \mathcal{I}\left[\left[x^{-3}\right]\left(\gamma_{-}^{0} \varphi_{+}^{1}+\gamma_{+}^{0} \varphi_{-}^{1}+\varphi_{+}^{1} \varphi_{-}^{1}\right)+\left[x^{-3}\right] \varphi^{1}\left(\gamma_{+}^{0}+\varphi_{+}^{1}\right)\left(\gamma_{-}^{0}+\varphi_{-}^{1}\right)\right. \\
& \left.+\left[x^{-3}\right]\left(\gamma_{-}^{x} \psi_{+}^{1}+\gamma_{+}^{x} \psi_{-}^{1}+\psi_{+}^{1} \psi_{-}^{1}\right)+\left[x^{-3}\right] \varphi^{1}\left(\gamma_{+}^{x}+\psi_{+}^{1}\right)\left(\gamma_{-}^{x}+\psi_{-}^{1}\right)\right] .
\end{aligned}
$$

It is easy to see $\triangle \varphi^{2} \in \mathfrak{A}$. By Proposition 3.15

$$
\begin{aligned}
& \left\|X_{0}\right\| \ll\left(\left\|\mathrm{e}^{x} x^{\sigma-1}\right\|+\left\|\mathrm{e}^{-x} x^{-\sigma-1}\right\|\right) \Upsilon_{0}, \\
& \left\|I_{1}\right\| \ll\left(\left\|\mathrm{e}^{x} x^{\sigma-1}\right\|+\left\|\mathrm{e}^{-x} x^{-\sigma-1}\right\|\right)\left\|\varphi^{1}\right\|\left(\gamma_{0}^{*}+\Upsilon_{0}\right), \quad\left\|I_{2}\right\| \ll|x|^{-2}\left(\left\|\varphi^{1}\right\|+1\right)\left(\gamma_{0}^{*}+\Upsilon_{0}\right)
\end{aligned}
$$

with $\Upsilon_{0}=\left(\gamma_{1}^{*}+\left\|\varphi_{+}^{1}\right\|+\left\|\psi_{+}^{1}\right\|\right)\left(\left\|\varphi_{+}^{1}\right\|+\left\|\psi_{+}^{1}\right\|+\left\|\varphi_{-}^{1}\right\|+\left\|\psi_{-}^{1}\right\|\right)$, the implied constants depending on $\delta$ only. Substituting (5.5), we have, under (5.4), $\left\|\triangle \varphi^{2}\right\| \ll\left(\gamma_{1}^{*}+1\right) \varepsilon$ for $\left|\eta x^{-1}\right|,\left|\eta^{-1} x^{-1}\right|<\varepsilon$. It is easy to verify $\left(\mathrm{e}^{x} x^{\sigma}\right)^{ \pm 1} \triangle \varphi^{2} \in \mathfrak{A}$, and we have

$$
\begin{aligned}
\left\|\mathrm{e}^{x} x^{\sigma} \triangle \varphi^{2}\right\| \ll & \left\|\mathrm{e}^{x} x^{\sigma-1}\right\|\left(\gamma_{1}^{*}+\left\|\psi_{+}^{1}\right\|\right)\left(\left\|\mathrm{e}^{x} x^{\sigma} \varphi_{-}^{1}\right\|+\left\|\mathrm{e}^{x} x^{\sigma} \psi_{+}^{1}\right\|\right) \\
& +|x|^{-1}\left(\gamma_{1}^{*}+\left\|\varphi_{+}^{1}\right\|\right)\left(\left\|\varphi_{+}^{1}\right\|+\left\|\psi_{-}^{1}\right\|\right)+\left\|I_{3}\right\|+\left\|I_{4}\right\|+\left\|\mathrm{e}^{x} x^{\sigma} I_{2}\right\|
\end{aligned}
$$

with

$$
\begin{aligned}
& I_{3}=\mathrm{e}^{x} x^{\sigma} \mathcal{I}\left[\mathrm{e}^{x} x^{\sigma-2}(1)_{x} \varphi^{1}\left(\gamma_{-}^{0}+\varphi_{-}^{1}\right)\left(\gamma_{+}^{x}+\psi_{+}^{1}\right)\right], \\
& I_{4}=\mathrm{e}^{x} x^{\sigma} \mathcal{I}\left[\mathrm{e}^{-x} x^{-\sigma-2}(1)_{x} \varphi^{1}\left(\gamma_{+}^{0}+\varphi_{+}^{1}\right)\left(\gamma_{-}^{x}+\psi_{-}^{1}\right)\right]
\end{aligned}
$$

and $I_{2}$ given above. By Proposition 3.16

$$
\begin{aligned}
& \left\|I_{3}-\gamma_{-}^{0} \gamma_{+}^{x} \mathrm{e}^{x} x^{\sigma} \mathcal{I}\left[\mathrm{e}^{x} x^{\sigma-2}(1)_{x} \varphi^{1}\right]\right\| \\
& =\left\|\mathrm{e}^{x} x^{\sigma} \mathcal{I}\left[\mathrm{e}^{-x} x^{-\sigma-1}(1)_{x} \mathrm{e}^{x} x^{\sigma-1} \varphi^{1}\left(\gamma_{-}^{0} \mathrm{e}^{x} x^{\sigma} \psi_{+}^{1}+\gamma_{+}^{x} \mathrm{e}^{x} x^{\sigma} \varphi_{-}^{1}+\psi_{+}^{1} \mathrm{e}^{x} x^{\sigma} \varphi_{-}^{1}\right)\right]\right\| \\
& \ll\left\|\mathrm{e}^{x} x^{\sigma-1}\right\|\left\|\varphi^{1}\right\|\left(\gamma_{1}^{*}+\left\|\psi_{+}^{1}\right\|\right)\left(\left\|\mathrm{e}^{x} x^{\sigma} \psi_{+}^{1}\right\|+\left\|\mathrm{e}^{x} x^{\sigma} \varphi_{-}^{1}\right\|\right), \\
& \left\|I_{4}\right\| \ll|x|^{-1}\left\|\varphi^{1}\right\|\left(\gamma_{0}^{*}+\left(\gamma_{1}^{*}+\left\|\varphi_{+}^{1}\right\|\right)\left(\left\|\varphi_{+}^{1}\right\|+\left\|\psi_{-}^{1}\right\|\right)\right), \\
& \left\|\mathrm{e}^{x} x^{\sigma} I_{2}\right\| \ll|x|^{-1}\left\|\mathrm{e}^{x} x^{\sigma-1}\right\|\left(\left\|\varphi^{1}\right\|+1\right)\left(\gamma_{0}^{*}+\Upsilon_{0}\right) .
\end{aligned}
$$

By (5.3)

$$
\left\|\mathrm{e}^{x} x^{\sigma} \mathcal{I}\left[\mathrm{e}^{x} x^{\sigma-2}(1)_{x} \varphi^{1}\right]\right\| \ll \gamma_{0}^{*}\left(\left\|\mathrm{e}^{x} x^{\sigma-1}\right\|^{3}+|x|^{-1}\left\|\mathrm{e}^{x} x^{\sigma-1}\right\|\right)+\left(\gamma_{1}^{*}\right)^{2}|x|^{-2}\left\|\mathrm{e}^{x} x^{\sigma-1}\right\|^{2} .
$$

Summing up these estimates we get $\left\|\mathrm{e}^{x} x^{\sigma} \triangle \varphi^{2}\right\| \ll\left(\gamma_{0}^{*}+\gamma_{1}^{*}+1\right) \varepsilon$ under (5.4). Similarly for $\left\|\mathrm{e}^{-x} x^{-\sigma} \triangle \varphi^{2}\right\|$ we have the same inequality. We may verify that $\mathrm{e}^{-x} x^{-\sigma} \triangle \varphi_{+}^{2} \in \mathfrak{A}$ as well, and by analogous arguments we have

$$
\begin{aligned}
& \left\|\triangle \varphi_{+}^{2}\right\| \ll\left|\gamma_{+}^{0}\right|\left\|\triangle \varphi^{2}\right\|+\left\|\varphi^{2}\right\|\left\|\varphi_{+}^{1}\right\|+\left|\gamma_{+}^{x}\right|\left\|\mathrm{e}^{x} x^{\sigma} \triangle \varphi^{2}\right\|+\left\|\varphi^{2}\right\|\left\|\mathrm{e}^{x} x^{\sigma} \psi_{+}^{1}\right\|, \\
& \left\|\mathrm{e}^{-x} x^{-\sigma} \triangle \varphi_{+}^{2}\right\| \ll\left|\gamma_{+}^{0}\right|\left\|\mathrm{e}^{-x} x^{-\sigma} \triangle \varphi^{2}\right\|+\left\|\varphi^{2}\right\|\left\|\mathrm{e}^{-x} x^{-\sigma} \varphi_{+}^{1}\right\|+\left|\gamma_{+}^{x}\right|\left\|\triangle \varphi^{2}\right\|+\left\|\varphi^{2}\right\|\left\|\psi_{+}^{1}\right\|,
\end{aligned}
$$

where $\left\|\varphi^{2}\right\| \leq\left\|\varphi^{1}\right\|+\left\|\triangle \varphi^{2}\right\|$. Substitution of (5.5) and the estimates for $\left\|\triangle \varphi^{2}\right\|$, . obtained above yields $\left\|\triangle \varphi_{+}^{2}\right\|,\left\|\mathrm{e}^{-x} x^{-\sigma} \triangle \varphi_{+}^{2}\right\| \ll\left(\gamma_{0}^{*}+\gamma_{1}^{*}+1\right)\left(\gamma_{1}^{*}+1\right) \varepsilon$. Furthermore $\mathrm{e}^{x} x^{\sigma} \triangle \varphi_{-}^{2}$, $\left(\mathrm{e}^{x} x^{\sigma}\right)^{ \pm 1} \triangle \psi_{ \pm}^{2} \in \mathfrak{A}$, and for $\left\|\triangle \varphi_{-}^{2}\right\|,\left\|\mathrm{e}^{x} x^{\sigma} \triangle \varphi_{-}^{2}\right\|,\left\|\triangle \psi_{ \pm}^{2}\right\|,\left\|\left(\mathrm{e}^{x} x^{\sigma}\right)^{ \pm} \triangle \psi_{ \pm}^{2}\right\|$, we have the same estimates. As will be shown later $\left(\mathrm{e}^{x} x^{\sigma}\right)^{ \pm 1} \triangle \varphi^{j+1},\left(\mathrm{e}^{x} x^{\sigma}\right)^{\mp 1} \triangle \varphi_{ \pm}^{j+1},\left(\mathrm{e}^{x} x^{\sigma}\right)^{ \pm 1} \triangle \psi_{ \pm}^{j+1} \in \mathfrak{A}$ for $j \geq 2$ as well. Let us set

$$
\begin{aligned}
\Psi_{j}:= & \left\|\triangle \varphi^{j+1}\right\|+\left\|\mathrm{e}^{x} x^{\sigma} \triangle \varphi^{j+1}\right\|+\left\|\mathrm{e}^{-x} x^{-\sigma} \triangle \varphi^{j+1}\right\| \\
& +\left\|\triangle \varphi_{+}^{j+1}\right\|+\left\|\mathrm{e}^{-x} x^{-\sigma} \triangle \varphi_{+}^{j+1}\right\|+\left\|\triangle \psi_{+}^{j+1}\right\|+\left\|\mathrm{e}^{x} x^{\sigma} \triangle \psi_{+}^{j+1}\right\| \\
& +\left\|\triangle \varphi_{-}^{j+1}\right\|+\left\|\mathrm{e}^{x} x^{\sigma} \triangle \varphi_{-}^{j+1}\right\|+\left\|\triangle \psi_{-}^{j+1}\right\|+\left\|\mathrm{e}^{-x} x^{-\sigma} \triangle \psi_{-}^{j+1}\right\| .
\end{aligned}
$$

For $j=1$, as shown above, we have 
Lemma 5.2. If $x_{\infty}^{1}=x_{\infty}^{1}\left(B_{0}, B_{x}, B_{*}, \delta\right)$ is sufficiently large, then $\left(\mathrm{e}^{x} x^{\sigma}\right)^{ \pm 1} \triangle \varphi^{2},\left(\mathrm{e}^{x} x^{\sigma}\right)^{\mp 1} \triangle \varphi_{ \pm}^{2}$, $\left(\mathrm{e}^{x} x^{\sigma}\right)^{ \pm 1} \triangle \psi_{ \pm}^{2}$ also belong to $\mathfrak{A}$, and, under the condition (5.4) for every $(\mathbf{c}, \sigma) \in B_{0} \times B_{x} \times B_{*}$, we have

$$
\begin{aligned}
& \left\|\varphi^{1}\right\| \leq K_{0}\left(\gamma_{0}^{*}+1\right) \varepsilon, \quad\left\|\varphi_{ \pm}^{1}\right\|,\left\|\psi_{ \pm}^{1}\right\| \leq K_{0}\left(\gamma_{0}^{*}+1\right)\left(\gamma_{1}^{*}+1\right) \varepsilon \\
& \Psi_{1} \leq K_{0}\left(\gamma_{0}^{*}+\gamma_{1}^{*}+1\right)\left(\gamma_{1}^{*}+1\right) \varepsilon
\end{aligned}
$$

for $\left|\eta x^{-1}\right|,\left|\eta^{-1} x^{-1}\right|<\varepsilon, x \in \Sigma_{0}\left(x_{\infty}^{1}, \delta\right)$, where $K_{0} \geq 1$ is some positive number depending on $\delta$ only.

\subsection{2 $\Psi_{j}$ for $j \geq 2$}

In addition to (5.4) suppose that

$$
\begin{aligned}
& \left\|\varphi^{\nu}\right\| \leq 3 K_{0}\left(\gamma_{0}^{*}+\gamma_{1}^{*}+1\right)\left(\gamma_{1}^{*}+1\right) \varepsilon \leq 1, \quad\left\|\varphi_{ \pm}^{\nu}\right\|,\left\|\psi_{ \pm}^{\nu}\right\| \leq 1, \\
& \left(\mathrm{e}^{x} x^{\sigma}\right)^{ \pm 1} \triangle \varphi^{\nu},\left(\mathrm{e}^{x} x^{\sigma}\right)^{\mp 1} \triangle \varphi_{ \pm}^{\nu},\left(\mathrm{e}^{x} x^{\sigma}\right)^{ \pm 1} \triangle \psi_{ \pm}^{\nu} \in \mathfrak{A}, \\
& \Psi_{\nu-1} \leq(1 / 2) \Psi_{\nu-2} \quad \text { if } \nu \geq 3
\end{aligned}
$$

for $2 \leq \nu \leq j$. Lemma 5.2 implies that (5.6), (5.7) and (5.8) are valid for $j=2$ if $3 K_{0} r_{0} \leq 1$, since $\left\|\varphi^{2}\right\| \leq\left\|\varphi^{1}\right\|+\Psi_{1},\left\|\varphi_{ \pm}^{2}\right\| \leq\left\|\varphi_{ \pm}^{1}\right\|+\Psi_{1}$ and $\left\|\psi_{ \pm}^{2}\right\| \leq\left\|\psi_{ \pm}^{1}\right\|+\Psi_{1}$.

From (5.2) it follows that, for $j \geq 2$,

$$
\begin{aligned}
\left\|\triangle \varphi^{j+1}\right\| \leq & \left\|\mathrm{e}^{x} x^{\sigma-1}(1)_{x} \triangle \omega_{1}^{j}+\mathrm{e}^{-x} x^{-\sigma-1}(1)_{x} \triangle \omega_{2}^{j}\right\| \\
& +\left\|4 \mathcal{I}\left[\mathrm{e}^{x} x^{\sigma-2}(1)_{x} \triangle\left(\varphi^{j} \omega_{1}^{j}\right)-\mathrm{e}^{-x} x^{-\sigma-2}(1)_{x} \triangle\left(\varphi^{j} \omega_{2}^{j}\right)\right]\right\| \\
& +\left\|\mathcal{I}\left[x^{-3}\left([1] \triangle \chi_{1}^{j}+[1] \triangle \chi_{2}^{j}+[1] \triangle\left(\varphi^{j} \chi_{1}^{j}\right)+[1] \triangle\left(\varphi^{j} \chi_{2}^{j}\right)\right)\right]\right\|
\end{aligned}
$$

with $\omega_{1}^{j}=\left(\gamma_{-}^{0}+\varphi_{-}^{j}\right)\left(\gamma_{+}^{x}+\psi_{+}^{j}\right), \omega_{2}^{j}=\left(\gamma_{+}^{0}+\varphi_{+}^{j}\right)\left(\gamma_{-}^{x}+\psi_{-}^{j}\right), \chi_{1}^{j}=\left(\gamma_{+}^{0}+\varphi_{+}^{j}\right)\left(\gamma_{-}^{0}+\varphi_{-}^{j}\right)$, $\chi_{2}^{j}=\left(\gamma_{+}^{x}+\psi_{+}^{j}\right)\left(\gamma_{-}^{x}+\psi_{-}^{j}\right)$. Then, by (5.4) and (5.6), the first two parts on the right-hand side are, respectively,

$$
\begin{aligned}
\leq & \left\|\mathrm{e}^{x} x^{\sigma-1}\right\|\left(\left|\gamma_{-}^{0}\right|\left\|\triangle \psi_{+}^{j}\right\|+\left|\gamma_{+}^{x}\right|\left\|\triangle \varphi_{-}^{j}\right\|+\left\|\triangle\left(\varphi_{-}^{j} \psi_{+}^{j}\right)\right\|\right) \\
& +\left\|\mathrm{e}^{-x} x^{-\sigma-1}\right\|\left(\left|\gamma_{+}^{0}\right|\left\|\triangle \psi_{-}^{j}\right\|+\left|\gamma_{-}^{x}\right|\left\|\triangle \varphi_{+}^{j}\right\|+\left\|\triangle\left(\varphi_{+}^{j} \psi_{-}^{j}\right)\right\|\right) \\
\leq & \left(1+\gamma_{1}^{*}\right) \varepsilon\left(\left\|\triangle \varphi_{+}^{j}\right\|+\left\|\triangle \psi_{+}^{j}\right\|+\left\|\triangle \varphi_{-}^{j}\right\|+\left\|\triangle \psi_{-}^{j}\right\|\right) \leq\left(1+\gamma_{1}^{*}\right) \varepsilon \Psi_{j-1},
\end{aligned}
$$

and

$$
\begin{aligned}
\leq & L_{0}\left\|\mathrm{e}^{x} x^{\sigma-1}\right\|\left(\left|\gamma_{-}^{0} \gamma_{+}^{x}\right|\left\|\triangle \varphi^{j}\right\|+\left|\gamma_{0}^{-}\right|\left\|\triangle\left(\varphi^{j} \psi_{+}^{j}\right)\right\|+\left|\gamma_{+}^{x}\right|\left\|\triangle\left(\varphi^{j} \varphi_{-}^{j}\right)\right\|+\left\|\triangle\left(\varphi^{j} \varphi_{-}^{j} \psi_{+}^{j}\right)\right\|\right) \\
& +L_{0}\left\|\mathrm{e}^{-x} x^{-\sigma-1}\right\|\left(\left|\gamma_{+}^{0} \gamma_{-}^{x}\right|\left\|\triangle \varphi^{j}\right\|+\left|\gamma_{+}^{0}\right|\left\|\triangle\left(\varphi^{j} \psi_{-}^{j}\right)\right\|+\left|\gamma_{-}^{x}\right|\left\|\triangle\left(\varphi^{j} \varphi_{+}^{j}\right)\right\|\right. \\
& \left.+\left\|\triangle\left(\varphi^{j} \varphi_{+}^{j} \psi_{-}^{j}\right)\right\|\right) \\
\leq & 2 L_{0}\left(\gamma_{0}^{*}+\gamma_{1}^{*}+1\right) \varepsilon\left(\left\|\triangle \varphi^{j}\right\|+\left\|\triangle \varphi_{+}^{j}\right\|+\left\|\triangle \psi_{+}^{j}\right\|+\left\|\triangle \varphi_{-}^{j}\right\|+\left\|\triangle \psi_{-}^{j}\right\|\right) \\
\leq & 2 L_{0}\left(\gamma_{0}^{*}+\gamma_{1}^{*}+1\right) \varepsilon \Psi_{j-1}
\end{aligned}
$$

for $\left|\eta x^{-1}\right|,\left|\eta^{-1} x^{-1}\right|<\varepsilon, x \in \Sigma_{0}\left(x_{\infty}^{1}, \delta\right)$, since

$$
\left\|\triangle\left(\varphi^{j} \varphi_{-}^{j} \psi_{+}^{j}\right)\right\| \leq\left\|\varphi_{-}^{j}\right\|\left\|\psi_{+}^{j}\right\|\left\|\triangle \varphi^{j}\right\|+\left\|\varphi^{j-1}\right\|\left\|\psi_{+}^{j}\right\|\left\|\triangle \varphi_{-}^{j}\right\|+\left\|\varphi^{j-1}\right\|\left\|\varphi_{-}^{j-1}\right\|\left\|\triangle \psi_{+}^{j}\right\| .
$$

Here $L_{0} \geq K_{0}$ is some number depending on $\delta$ only, which may be retaken larger, if necessary, in each appearance below. Similarly the remaining part is $\leq 2 L_{0}\left(\gamma_{0}^{*}+\gamma_{1}^{*}+1\right) \varepsilon^{2} \Psi_{j-1}$, and hence

$$
\left\|\triangle \varphi^{j+1}\right\| \leq 5 L_{0}\left(\gamma_{0}^{*}+\gamma_{1}^{*}+1\right) \varepsilon \Psi_{j-1} .
$$


Observe that $\mathrm{e}^{x} x^{\sigma} \mathcal{I}\left[\mathrm{e}^{x} x^{\sigma-2}(1)_{x} \triangle\left(\varphi^{j} \omega_{1}^{j}\right)\right]=\mathrm{e}^{x} x^{\sigma} \mathcal{I}\left[\mathrm{e}^{-x} x^{-\sigma-1}(1)_{x} \mathrm{e}^{x} x^{\sigma-1}(\cdots)\right]$, where

$$
\begin{aligned}
(\cdots)= & \mathrm{e}^{x} x^{\sigma} \triangle\left(\varphi^{j} \omega_{1}^{j}\right)=\left(\gamma_{-}^{0} \gamma_{+}^{x}+\gamma_{-}^{0} \psi_{+}^{j}+\gamma_{+}^{x} \varphi_{-}^{j}+\psi_{+}^{j} \varphi_{-}^{j}\right) \cdot \mathrm{e}^{x} x^{\sigma} \triangle \varphi^{j} \\
& +\left(\gamma_{-}^{0}+\varphi_{-}^{j}\right) \varphi^{j-1} \cdot \mathrm{e}^{x} x^{\sigma} \triangle \psi_{+}^{j}+\left(\gamma_{+}^{x}+\psi_{+}^{j-1}\right) \varphi^{j-1} \cdot \mathrm{e}^{x} x^{\sigma} \triangle \varphi_{-}^{j} .
\end{aligned}
$$

By Proposition 3.16, this and analogous facts combined with (5.7) imply that $\mathrm{e}^{x} x^{\sigma} \triangle \varphi^{j+1} \in \mathfrak{A}$. Then, dividing $\left\|\mathrm{e}^{x} x^{\sigma} \triangle \varphi^{j+1}\right\|$ into three parts corresponding to those of $\left\|\triangle \varphi^{j+1}\right\|$ above, we derive

$$
\begin{aligned}
\left\|\mathrm{e}^{x} x^{\sigma} \triangle \varphi^{j+1}\right\| \leq & 4 L_{0}\left(\gamma_{0}^{*}+\gamma_{1}^{*}+1\right) \varepsilon\left(\left\|\triangle \varphi^{j}\right\|+\left\|\mathrm{e}^{x} x^{\sigma} \triangle \varphi^{j}\right\|\right. \\
& \left.+\left\|\triangle \varphi_{+}^{j}\right\|+\left\|\mathrm{e}^{x} x^{\sigma} \triangle \psi_{+}^{j}\right\|+\left\|\mathrm{e}^{x} x^{\sigma} \triangle \varphi_{-}^{j}\right\|+\left\|\triangle \psi_{-}^{j}\right\|\right) \\
\leq & 4 L_{0}\left(\gamma_{0}^{*}+\gamma_{1}^{*}+1\right) \varepsilon \Psi_{j-1} .
\end{aligned}
$$

Similarly we may show that $\mathrm{e}^{-x} x^{-\sigma} \triangle \varphi^{j+1} \in \mathfrak{A}$ and

$$
\left\|\mathrm{e}^{-x} x^{-\sigma} \triangle \varphi^{j+1}\right\| \leq 4 L_{0}\left(\gamma_{0}^{*}+\gamma_{1}^{*}+1\right) \varepsilon \Psi_{j-1} .
$$

Since $\mathrm{e}^{-x} x^{-\sigma} \mathcal{I}\left[x^{-1}(1)_{x}\left(\varphi^{j+1}\left(\gamma_{+}^{0}+\varphi_{+}^{j}\right)-\varphi^{j}\left(\gamma_{+}^{0}+\varphi_{+}^{j-1}\right)\right)\right]=\mathrm{e}^{-x} x^{-\sigma} \mathcal{I}\left[\mathrm{e}^{x} x^{\sigma-1}(1)_{x}(\cdots)\right]$ with $(\cdots)=\left(\gamma_{+}^{0}+\varphi_{+}^{j}\right) \mathrm{e}^{-x} x^{-\sigma} \triangle \varphi^{j+1}+\varphi^{j} \cdot \mathrm{e}^{-x} x^{-\sigma} \triangle \varphi_{+}^{j}$, we have $\mathrm{e}^{-x} x^{-\sigma} \triangle \varphi_{+}^{j+1} \in \mathfrak{A}$ as well. Furthermore by $(5.6)$

$$
\begin{aligned}
\left\|\triangle \varphi_{+}^{j+1}\right\| & \leq L_{0}\left(\gamma_{1}^{*}\left\|\triangle \varphi^{j+1}\right\|+\left\|\triangle\left(\varphi^{j+1} \varphi_{+}^{j}\right)\right\|+\gamma_{1}^{*}\left\|\mathrm{e}^{x} x^{\sigma} \triangle \varphi^{j+1}\right\|+\left\|\mathrm{e}^{x} x^{\sigma} \triangle\left(\varphi^{j+1} \psi_{+}^{j}\right)\right\|\right) \\
& \leq L_{0}\left(\gamma_{1}^{*}+1\right)\left(\left\|\triangle \varphi^{j+1}\right\|+\left\|\mathrm{e}^{x} x^{\sigma} \triangle \varphi^{j+1}\right\|\right. \\
& \left.+3 K_{0}\left(\gamma_{0}^{*}+\gamma_{1}^{*}+1\right) \varepsilon\left(\left\|\triangle \varphi_{+}^{j}\right\|+\left\|\mathrm{e}^{x} x^{\sigma} \triangle \psi_{+}^{j}\right\|\right)\right)
\end{aligned}
$$

since

$$
\begin{aligned}
& \left\|\triangle\left(\varphi^{j+1} \varphi_{+}^{j}\right)\right\| \leq\left\|\varphi_{+}^{j}\right\|\left\|\triangle \varphi^{j+1}\right\|+\left\|\varphi^{j}\right\|\left\|\triangle \varphi_{+}^{j}\right\|, \\
& \left\|\mathrm{e}^{x} x^{\sigma} \triangle\left(\varphi^{j+1} \psi_{+}^{j}\right)\right\| \leq\left\|\psi_{+}^{j}\right\|\left\|\mathrm{e}^{x} x^{\sigma} \triangle \varphi^{j+1}\right\|+\left\|\varphi^{j}\right\|\left\|\mathrm{e}^{x} x^{\sigma} \triangle \psi_{+}^{j}\right\|,
\end{aligned}
$$

and similarly

$$
\begin{aligned}
\left\|\mathrm{e}^{-x} x^{-\sigma} \triangle \varphi_{+}^{j+1}\right\| \leq & L_{0}\left(\gamma_{1}^{*}+1\right)\left(\left\|\triangle \varphi^{j+1}\right\|+\left\|\mathrm{e}^{-x} x^{-\sigma} \triangle \varphi^{j+1}\right\|\right. \\
& \left.+3 K_{0}\left(\gamma_{0}^{*}+\gamma_{1}^{*}+1\right) \varepsilon\left(\left\|\mathrm{e}^{-x} x^{-\sigma} \triangle \varphi_{+}^{j}\right\|+\left\|\triangle \psi_{+}^{j}\right\|\right)\right) .
\end{aligned}
$$

We combine these estimates with (5.9), (5.10) and (5.11) to obtain

$$
\begin{aligned}
& \left\|\triangle \varphi_{+}^{j+1}\right\|+\left\|\mathrm{e}^{-x} x^{-\sigma} \triangle \varphi_{+}^{j+1}\right\| \leq 3 L_{0}^{2}\left(\gamma_{0}^{*}+\gamma_{1}^{*}+1\right)\left(\gamma_{1}^{*}+1\right) \varepsilon \Psi_{j-1} \\
& \quad+L_{0}\left(\gamma_{1}^{*}+1\right)\left(2\left\|\triangle \varphi^{j+1}\right\|+\left\|\mathrm{e}^{x} x^{\sigma} \triangle \varphi^{j+1}\right\|+\left\|\mathrm{e}^{-x} x^{-\sigma} \triangle \varphi^{j+1}\right\|\right) \\
& \leq 21 L_{0}^{2}\left(\gamma_{0}^{*}+\gamma_{1}^{*}+1\right)\left(\gamma_{1}^{*}+1\right) \varepsilon \Psi_{j-1} .
\end{aligned}
$$

The other differences $\triangle \varphi_{-}^{j+1}, \mathrm{e}^{x} x^{\sigma} \triangle \varphi_{-}^{j+1}, \triangle \psi_{ \pm}^{j+1},\left(\mathrm{e}^{x} x^{\sigma}\right)^{ \pm 1} \triangle \psi_{ \pm}^{j+1}$ are treated in a similar manner. Thus we have shown that (5.7) is valid for $\nu \leq j+1$, and that $\Psi_{j} \leq 100 L_{0}^{2}\left(\gamma_{0}^{*}+\gamma_{1}^{*}+1\right)\left(\gamma_{1}^{*}+\right.$ 1) $\varepsilon \Psi_{j-1}$. Choose $r_{0}$ in (5.4) in such a way that $3 K_{0} r_{0} \leq 100 L_{0}^{2} r_{0} \leq 1 / 2$. Then $\Psi_{j} \leq(1 / 2) \Psi_{j-1}$, and hence (5.8) is valid for $\nu \leq j+1$. By Lemma 5.2

$$
\begin{aligned}
\left\|\varphi^{j+1}\right\| & \leq\left\|\varphi^{1}\right\|+\sum_{\nu=1}^{j}\left\|\Delta \varphi^{\nu+1}\right\| \leq\left\|\varphi^{1}\right\|+\sum_{\nu=1}^{j} \Psi_{\nu} \leq\left\|\varphi^{1}\right\|+2 \Psi_{1} \\
& \leq K_{0}\left(\gamma_{0}^{*}+1\right) \varepsilon+2 K_{0}\left(\gamma_{0}^{*}+\gamma_{1}^{*}+1\right)\left(\gamma_{1}^{*}+1\right) \varepsilon \leq 3 K_{0}\left(\gamma_{0}^{*}+\gamma_{1}^{*}+1\right)\left(\gamma_{1}^{*}+1\right) \varepsilon, \\
\left\|\varphi_{+}^{j+1}\right\| & \leq\left\|\varphi_{+}^{1}\right\|+2 \Psi_{1} \leq 3 K_{0}\left(\gamma_{0}^{*}+\gamma_{1}^{*}+1\right)\left(\gamma_{1}^{*}+1\right) \varepsilon, \quad \ldots,
\end{aligned}
$$

that is, (5.6) is also valid for $\nu \leq j+1$. Thus we have shown that (5.6), (5.7) and (5.8) are valid for every $\nu$ if $r_{0}$ is as above. 
Proposition 5.3. For $j \geq 2$ we have $\left(\mathrm{e}^{x} x^{\sigma}\right)^{ \pm 1} \triangle \varphi^{j},\left(\mathrm{e}^{x} x^{\sigma}\right)^{\mp 1} \triangle \varphi_{ \pm}^{j},\left(\mathrm{e}^{x} x^{\sigma}\right)^{ \pm 1} \triangle \psi_{ \pm}^{j} \in \mathfrak{A}$, and $\Psi_{j} \leq(1 / 2) \Psi_{j-1}$ for $\left|\eta x^{-1}\right|,\left|\eta^{-1} x^{-1}\right|<\varepsilon, x \in \Sigma_{0}\left(x_{\infty}^{1}, \delta\right)$ under (5.4) with $r_{0}=r_{0}(\delta)$ such that $100 L_{0}^{2} r_{0} \leq 1 / 2$.

\subsection{Asymptotic coefficients}

Let $\phi \in \hat{\mathfrak{A}}$ be given by

$$
\phi=\sum_{n=1}^{\infty} p_{n}^{+}(x)\left(\mathrm{e}^{x} x^{\sigma-1}\right)^{n}+\sum_{n=1}^{\infty} p_{n}^{-}(x)\left(\mathrm{e}^{-x} x^{-\sigma-1}\right)^{n}+p_{0}(x) x^{-1} .
$$

For every $p_{n}^{+}(x) \nsim 0$, let $d_{+}(n) \in \mathbb{N} \cup\{0\}$ be such that $p_{n}^{+}(x)=x^{-d_{+}(n)}\left(a_{n}^{+}+O\left(x^{-1}\right)\right)$ with $a_{n}^{+} \neq 0$, and assign the lattice point $\left(n,-d_{+}(n)\right) \in \mathbb{Z}^{2}$ to $p_{n}^{+}(x)$. For the other asymptotic coefficients $p_{0}(x), p_{n}^{-}(x) \nsim 0$, the degrees $d(0)$ and $d_{-}(n)$ are similarly defined, and the lattice points $(0,-d(0))$ and $\left(-n,-d_{-}(n)\right)$ are assigned to $p_{0}(x) \nsim 0$ and to $p_{n}^{-}(x) \nsim 0$, respectively. Then denote by $\varpi(\phi)$ the set of such lattice points for all asymptotic coefficients $\nsim 0$ of $\phi$. For $d, m_{-}, m_{+} \in \mathbb{Z}$ satisfying $m_{-} \leq m_{+}, d \geq 0$, set

$$
\left[m_{-}, m_{+} ;-d\right]:=\left\{\left(x_{1}, x_{2}\right) \in \mathbb{Z}^{2} ; x_{2} \leq-d, x_{2} \leq x_{1}-m_{-}-d, x_{2} \leq-x_{1}+m_{+}-d\right\} .
$$

Then, for $\varphi^{j}, \varphi_{ \pm}^{j}, \psi_{ \pm}^{j}$ given by (5.2), we have

Proposition 5.4. For every $j \geq 2$, the lattice sets $\varpi\left(\varphi^{j-1}\right), \varpi\left(\varphi_{ \pm}^{j-1}\right), \varpi\left(\psi_{ \pm}^{j-1}\right)$ consist of finite numbers of lattice points, and have the properties:

$$
\begin{aligned}
& \varpi\left(\varphi^{j}\right) \subset[-1,1 ; 0], \quad \varpi\left(\triangle \varphi^{j}\right) \subset[-j, j ;-j+1] ; \\
& \varpi\left(\varphi_{+}^{j}\right), \varpi\left(\psi_{-}^{j}\right) \subset[0,2 ; 0], \quad \varpi\left(\triangle \varphi_{+}^{j}\right), \varpi\left(\triangle \psi_{-}^{j}\right) \subset[-j,-1 ;-j] \cup[0, j+1 ;-j+1] ; \\
& \varpi\left(\varphi_{-}^{j}\right), \varpi\left(\psi_{+}^{j}\right) \subset[-2,0 ; 0], \quad \varpi\left(\triangle \varphi_{-}^{j}\right), \varpi\left(\triangle \psi_{+}^{j}\right) \subset[-j-1,0 ;-j+1] \cup[1, j ;-j] .
\end{aligned}
$$

The polygons packing the lattice sets on the right-hand sides are described in Fig. 5.1.
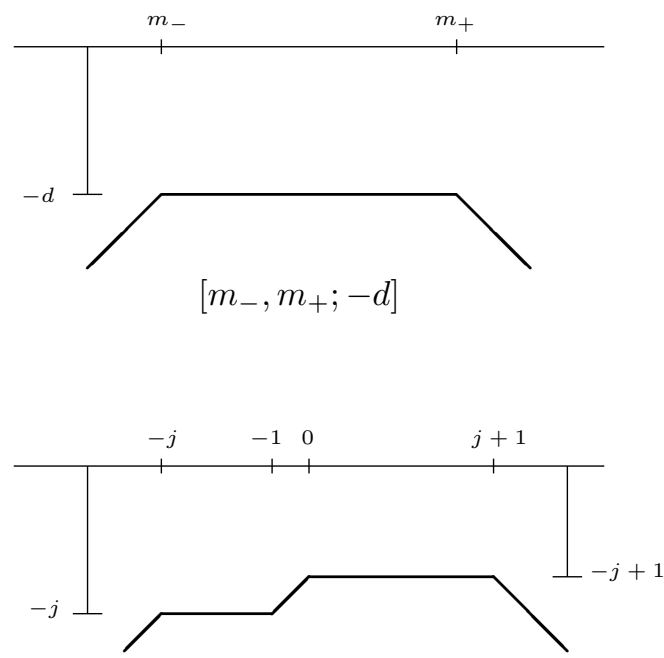

$$
[-j,-1 ;-j] \cup[0, j+1 ;-j+1]
$$
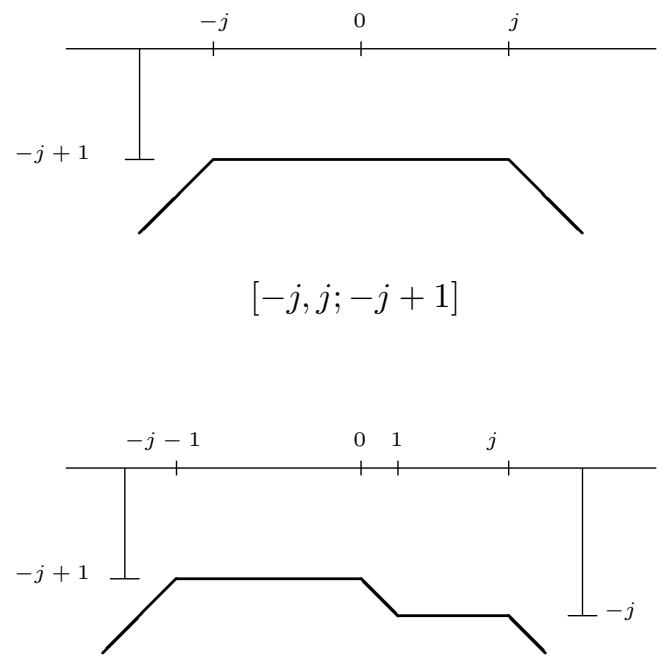

$$
[-j-1,0 ;-j+1] \cup[1, j ;-j]
$$

Figure 5.1. Polygons packing the lattice sets. 
Proof. Let us identify $\left(\mathrm{e}^{x} x^{\sigma-1}\right)^{n} x^{-d_{+}(n)}, x^{-1} x^{-d(0)}$ and $\left(\mathrm{e}^{-x} x^{-\sigma-1}\right)^{n} x^{-d_{-}(n)}$ with $\left(n,-d_{+}(n)\right)$, $(0,-d(0))$ and $\left(n,-d_{-}(n)\right)$, respectively. Then we may canonically define the product of them and write, for example, $\left(\mathrm{e}^{x} x^{\sigma-1}\right)^{n} x^{-d_{+}(n)}\left(-n^{\prime},-d_{-}\left(n^{\prime}\right)\right)=\left(n,-d_{+}(n)\right)\left(-n^{\prime},-d_{-}\left(n^{\prime}\right)\right)=(n-$ $\left.n^{\prime},-d_{+}(n)-d_{-}\left(n^{\prime}\right)-2 n^{\prime}\right)$ if $n>n^{\prime},\left(\mathrm{e}^{x} x^{\sigma-1}\right)^{n} x^{-d_{+}(n)}\left(-n,-d_{-}(n)\right)=\left(0,-d_{+}(n)-d_{-}(n)-\right.$ $2 n+1)$. The following formulas are easily obtained:

$$
\begin{aligned}
& \mathrm{e}^{x} x^{\sigma-1}[-j, j ;-j+1]=[-j+1,-1 ;-j-1] \cup[0,1 ;-j] \cup[2, j+1 ;-j+1], \\
& \mathrm{e}^{x} x^{\sigma-1}([-j-1,0 ;-j+1] \cup[1, j ;-j])=[-j,-1 ;-j-1] \cup[0, j+1 ;-j],
\end{aligned}
$$

in particular,

$$
\mathrm{e}^{x} x^{\sigma-1}[-1,1 ; 0] \subset[1,2 ; 0], \quad \mathrm{e}^{x} x^{\sigma-1}[-2,0 ; 0] \subset[0,2 ;-1] .
$$

Indeed, for example, (5.12) is verified by using

$$
[-j, j ;-j+1]=\left.\left.\left.[-j,-2 ;-j+1]\right|_{x_{1} \leq-2} \cup[-1,0 ;-j+1]\right|_{-1 \leq x_{1} \leq 0} \cup[1, j ;-j+1]\right|_{x_{1} \geq 1} .
$$

We show the relations by induction on $j$. By virtue of the symmetric property of $(5.2)$, it is sufficient to focus on $\varphi^{j}$ and $\varphi_{+}^{j}$. By (5.3), $\left(\varphi^{1}\right) \subset[-1,1 ; 0], \varpi\left(\varphi_{+}^{1}\right), \varpi\left(\psi_{-}^{1}\right) \subset[0,2 ; 0]$, $\varpi\left(\varphi_{-}^{1}\right), \varpi\left(\psi_{+}^{1}\right) \subset[-2,0 ; 0]$. Note that $\mathcal{I}\left[\left(\mathrm{e}^{x} x^{\sigma-1}\right)^{m}\left[x^{-l}\right]\right]=\left(\mathrm{e}^{x} x^{\sigma-1}\right)^{m}\left[x^{-l}\right], \mathcal{I}\left[x^{-1}\left[x^{-l-1}\right]\right]=$ $\left[x^{-l-1}\right], \mathcal{I}\left[\left(\mathrm{e}^{-x} x^{-\sigma-1}\right)^{m}\left[x^{-l}\right]\right]=\left(\mathrm{e}^{-x} x^{-\sigma-1}\right)^{m}\left[x^{-l}\right]$, where $m \in \mathbb{N}, l \in \mathbb{N} \cup\{0\}$. By $\triangle \varphi^{2}=$ $F_{0}\left(x, \varphi^{1}, \varphi_{+}^{1}, \psi_{+}^{1}, \varphi_{-}^{1}, \psi_{-}^{1}\right)-F_{0}(x, 0,0,0,0,0)$ and $(5.14)$ we have $\varpi\left(\triangle \varphi^{2}\right) \subset[-2,2 ;-1]$, and hence $\varpi\left(\varphi^{2}\right) \subset[-1,1 ; 0] \cup[-2,2 ;-1]=[-1,1 ; 0]$. In obtaining this, we have used $\varpi\left(\mathrm{e}^{x} x^{\sigma-2} \varphi_{-}^{1} \varphi^{1}\right)$ $\subset x^{-1} \mathrm{e}^{x} x^{\sigma-1}[-2,0 ; 0][-1,1 ; 0] \subset[0,2 ;-2][-1,1 ; 0] \subset[-1,3 ;-2] \subset[0,2 ;-1]$. From

$$
\triangle \varphi_{+}^{2}=\mathcal{I}\left[x^{-1}[1]\left(\left([1]+\varphi_{+}^{1}\right) \triangle \varphi^{2}+\varphi^{1} \varphi_{+}^{1}\right)+\mathrm{e}^{x} x^{\sigma-1}[1]\left(\left([1]+\psi_{+}^{1}\right) \triangle \varphi^{2}+\varphi^{1} \psi_{+}^{1}\right)\right],
$$

we derive $\varpi\left(\triangle \varphi_{+}^{2}\right) \subset[-2,-1 ;-2] \cup[0,3 ;-1]$ and $\varpi\left(\varphi_{+}^{2}\right) \subset[0,2 ; 0]$ by using

$$
\begin{aligned}
& \varpi\left(\mathrm{e}^{x} x^{\sigma-1} \triangle \varphi^{2}\right) \subset \mathrm{e}^{x} x^{\sigma-1}[-2,2 ;-1] \subset \mathrm{e}^{x} x^{\sigma-1}([-2,-2 ;-1] \cup[-1,0 ;-1] \cup[1,2 ;-1]) \\
& \subset {[-1,-1 ;-3] \cup[0,1 ;-2] \cup[2,3 ;-1] \subset[0,1 ;-2] \cup[2,3 ;-1] \subset[0,3 ;-1], } \\
& \varpi\left(\mathrm{e}^{x} x^{\sigma-1} \psi_{+}^{1} \triangle \varphi^{2}\right) \subset \psi_{+}^{1} \varpi\left(\mathrm{e}^{x} x^{\sigma-1} \triangle \varphi^{2}\right) \subset[-2,0 ; 0]([0,1 ;-2] \cup[2,3 ;-1]) \\
& \subset[-2,1 ;-2] \cup[0,3 ;-1] \subset[-2,-1 ;-2] \cup[0,3 ;-1], \\
& \varpi\left(\mathrm{e}^{x} x^{\sigma-1} \varphi^{1} \psi_{+}^{1}\right) \subset \mathrm{e}^{x} x^{\sigma-1}[-2,0 ; 0][-1,1 ; 0] \subset[0,2 ;-1]([-1,-1 ; 0] \cup[0,1 ; 0]) \\
& \subset {[-1,1 ;-2] \cup[0,3 ;-1] }
\end{aligned}
$$

and so on. Hence the assertion is valid for $j=2$.

Suppose that the assertion is valid for every integer $\leq j$. From (5.2) it follows that

$$
\begin{aligned}
\triangle \varphi^{j+1}= & \mathrm{e}^{x} x^{\sigma-1}[1]\left(\left([1]+\varphi_{-}^{j}\right) \triangle \psi_{+}^{j}+\left([1]+\psi_{+}^{j-1}\right) \triangle \varphi_{-}^{j}\right)+\mathrm{e}^{-x} x^{-\sigma-1}(\cdots) \\
& +\mathcal{I}\left[\mathrm { e } ^ { x } x ^ { \sigma - 2 } [ 1 ] \left(\left([1]+\varphi_{-}^{j}\right)\left([1]+\psi_{+}^{j}\right) \triangle \varphi^{j}+\left([1]+\varphi_{-}^{j}\right) \varphi^{j-1} \triangle \psi_{+}^{j}\right.\right. \\
& \left.\left.+\left([1]+\psi_{+}^{j-1}\right) \varphi^{j-1} \triangle \varphi_{-}^{j}\right)+\mathrm{e}^{-x} x^{-\sigma-2}(\cdots)\right] \\
& +\mathcal{I}\left[x ^ { - 3 } \left([1]\left([1]+\varphi_{+}^{j}\right)\left([1]+\varphi_{-}^{j}\right) \triangle \varphi^{j}+\left([1]+[1] \varphi^{j-1}\right)\left([1]+\varphi_{-}^{j}\right) \triangle \varphi_{+}^{j}\right.\right. \\
& \left.\left.+\left([1]+[1] \varphi^{j-1}\right)\left([1]+\varphi_{+}^{j-1}\right) \triangle \varphi_{-}^{j}\right)+x^{-3}(\cdots)\right] .
\end{aligned}
$$

By (5.12) and (5.13) we have

$$
\begin{aligned}
& \varpi\left(\mathrm{e}^{x} x^{\sigma-2} \triangle \varphi^{j}\right) \subset[-j+1,-1 ;-j-2] \cup[0,1 ;-j-1] \cup[2, j+1 ;-j] \\
& \subset[-j-1, j+1 ;-1] \\
& \varpi\left(\mathrm{e}^{x} x^{\sigma-2} \varphi^{j-1} \triangle \varphi_{-}^{j}\right) \subset[-1,1 ; 0]([-j,-1 ;-j-2] \cup[0, j+1 ;-j-1]) \\
& \subset[-j-1,0 ;-j-2] \cup[-1, j+2 ;-j-1] \subset[-j-1, j+1 ;-j], \\
& \varpi\left(\mathrm{e}^{x} x^{\sigma-2} \varphi^{j-1} \psi_{+}^{j} \triangle \varphi_{-}^{j}\right) \subset[-2,0 ; 0]([-j-1,0 ;-j-2] \cup[-1, j+2 ;-j-1]) \\
& \subset[-j-1, j+1 ;-j]
\end{aligned}
$$


and so on. From these it follows that $\varpi\left(\triangle \varphi^{j+1}\right) \subset[-j-1, j+1 ;-j]$ and consequently $\varpi\left(\varphi^{j+1}\right) \subset$ $[-1,1 ; 0]$. Furthermore,

$$
\begin{aligned}
\triangle \varphi_{+}^{j+1}= & \mathcal{I}\left[x^{-1}[1]\left(\left([1]+\varphi_{+}^{j}\right) \triangle \varphi^{j+1}+\varphi^{j} \triangle \varphi_{+}^{j}\right)\right. \\
& \left.+\mathrm{e}^{x} x^{\sigma-1}[1]\left(\left([1]+\psi_{+}^{j}\right) \triangle \varphi^{j+1}+\varphi^{j} \triangle \psi_{+}^{j}\right)\right] .
\end{aligned}
$$

Observing that, by (5.13),

$$
\begin{aligned}
& \varpi\left(\mathrm{e}^{x} x^{\sigma-1} \varphi^{j} \triangle \psi_{+}^{j}\right) \subset[-1,1 ; 0]([-j,-1 ;-j-1] \cup[0, j+1 ;-j]) \\
& \quad \subset[-j-1,0 ;-j-1] \cup[-1,1 ; 0][0, j+1 ;-j] \subset[-j-1, j ;-j-1] \cup[0, j+2 ;-j],
\end{aligned}
$$

where $[-1,1 ; 0][0, j+1 ;-j]=([-1,-1 ; 0] \cup[0,1 ; 0])[0, j+1 ;-j] \subset[-1, j ;-j-1] \cup[0, j+2 ;-j]$, and so on, we have $\varpi\left(\triangle \varphi_{+}^{j+1}\right) \subset[-j-1,-1 ;-j-1] \cup[0, j+2 ;-j]$ and $\varpi\left(\varphi_{+}^{j+1}\right) \subset[0,2 ; 0]$. Thus we obtain the proposition.

Proposition 5.5. The summands of $\varphi^{j}, \varphi_{ \pm}^{j}$ and $\psi_{ \pm}^{j}$ satisfy the following: if $n \geq 1$,

(i) $p_{n}^{+}(x)=\left(\gamma_{-}^{0} \gamma_{+}^{x}\right)^{n}[1], p_{n}^{-}(x)=\left(\gamma_{+}^{0} \gamma_{-}^{x}\right)^{n}[1]$ for $\varphi^{j}$;

(ii) $p_{n}^{+}(x)=\gamma_{+}^{x}\left(\gamma_{-}^{0} \gamma_{+}^{x}\right)^{n-1}[1], p_{n}^{-}(x)=\gamma_{+}^{0}\left(\gamma_{+}^{0} \gamma_{-}^{x}\right)^{n}[1]$ for $\varphi_{+}^{j}$;

(iii) $p_{n}^{+}(x)=\gamma_{+}^{x}\left(\gamma_{-}^{0} \gamma_{+}^{x}\right)^{n}[1], p_{n}^{-}(x)=\gamma_{+}^{0}\left(\gamma_{+}^{0} \gamma_{-}^{x}\right)^{n-1}[1]$ for $\psi_{+}^{j}$;

(iv) $p_{n}^{+}(x)=\gamma_{-}^{0}\left(\gamma_{-}^{0} \gamma_{+}^{x}\right)^{n}[1], p_{n}^{-}(x)=\gamma_{-}^{x}\left(\gamma_{+}^{0} \gamma_{-}^{x}\right)^{n-1}[1]$ for $\varphi_{-}^{j}$;

(v) $p_{n}^{+}(x)=\gamma_{-}^{0}\left(\gamma_{-}^{0} \gamma_{+}^{x}\right)^{n-1}[1], p_{n}^{-}(x)=\gamma_{-}^{x}\left(\gamma_{+}^{0} \gamma_{-}^{x}\right)^{n}[1]$ for $\psi_{-}^{j}$.

Furthermore $p_{0}(x)=\gamma_{ \pm}^{0}[1]$ for $\varphi_{ \pm}$, and $p_{0}(x)=\gamma_{ \pm}^{x}[1]$ for $\psi_{ \pm}$.

Proof. Note that the relations for $\varphi^{j+1}, \varphi_{+}^{j+1}$ and $\psi_{-}^{j+1}$ in (5.2) are rewritten in the form

$$
\begin{aligned}
& \varphi^{j+1}=F_{0}\left(x, \varphi^{j}, \mathrm{e}^{x} x^{\sigma} \varphi_{+*}^{j}, \psi_{+}^{j}, \varphi_{-}^{j}, \mathrm{e}^{x} x^{\sigma} \psi_{-*}^{j}\right), \\
& \varphi_{+*}^{j+1}=\mathrm{e}^{-x} x^{-\sigma} F_{+}\left(x, \varphi^{j+1}, \mathrm{e}^{x} x^{\sigma} \varphi_{+*}^{j}, \psi_{+}^{j}\right), \\
& \psi_{-*}^{j+1}=\mathrm{e}^{-x} x^{-\sigma} G_{-}\left(x, \varphi^{j+1}, \varphi_{-}^{j}, \mathrm{e}^{x} x^{\sigma} \psi_{-*}^{j}\right),
\end{aligned}
$$

where $\varphi_{+*}^{j}=\mathrm{e}^{-x} x^{-\sigma} \varphi_{+}^{j}, \psi_{-*}^{j}=\mathrm{e}^{-x} x^{-\sigma} \psi_{-}^{j}$. Combining these with the relations for $\varphi_{-}^{j+1}$ and $\psi_{+}^{j+1}$ in (5.2), by induction on $j$ we may verify the facts: if $n \geq 0$,

(a) $p_{n}^{+}(x)=\left(\gamma_{-}^{0} \gamma_{+}^{x}\right)^{n}[1]$ for $\varphi^{j}$;

(b) $p_{n}^{+}(x)=\gamma_{+}^{x}\left(\gamma_{-}^{0} \gamma_{+}^{x}\right)^{n}[1]$ for $\varphi_{+*}^{j}, \psi_{+}^{j}$;

(c) $p_{n}^{+}(x)=\gamma_{-}^{0}\left(\gamma_{-}^{0} \gamma_{+}^{x}\right)^{n}[1]$ for $\varphi_{-}^{j}, \psi_{-*}^{j}$.

Similarly, if $n \geq 0$,

$\left(\mathrm{a}^{\prime}\right) p_{n}^{-}(x)=\left(\gamma_{+}^{0} \gamma_{-}^{x}\right)^{n}[1]$ for $\varphi^{j}$

$\left(\mathrm{b}^{\prime}\right) p_{n}^{-}(x)=\gamma_{+}^{0}\left(\gamma_{+}^{0} \gamma_{-}^{x}\right)^{n}[1]$ for $\varphi_{+}^{j}, \psi_{+*}^{j}$;

$\left(\mathrm{c}^{\prime}\right) p_{n}^{-}(x)=\gamma_{-}^{x}\left(\gamma_{+}^{0} \gamma_{-}^{x}\right)^{n}[1]$ for $\varphi_{-*}^{j}, \psi_{-}^{j}$,

where $\psi_{+*}^{j}=\mathrm{e}^{x} x^{\sigma} \psi_{+}^{j}, \varphi_{-*}^{j}=\mathrm{e}^{x} x^{\sigma} \varphi_{-}^{j}$. Then the proposition immediately follows. 


\subsection{Completion of the proof of Theorem 2.1}

By Proposition 5.4, for $j \geq 2,\left\|\triangle \varphi^{j}\right\|,\left\|\triangle \varphi_{ \pm}^{j}\right\|,\left\|\triangle \psi_{ \pm}^{j}\right\| \ll|x|^{-j+1}$, and hence $\left\|\left(\mathrm{e}^{x} x^{\sigma}\right)^{ \pm 1} \triangle \varphi^{j}\right\|$, $\left\|\left(\mathrm{e}^{x} x^{\sigma}\right)^{\mp 1} \triangle \varphi_{ \pm}^{j}\right\|,\left\|\left(\mathrm{e}^{x} x^{\sigma}\right)^{ \pm 1} \triangle \psi_{ \pm}^{j}\right\| \ll|x|^{-j+2}$ if $\left|\eta x^{-1}\right|,\left|\eta^{-1} x^{-1}\right|<\varepsilon, x \in \Sigma_{0}\left(x_{\infty}^{1}, \delta\right)$, the implied constants possibly depending on $j$. Let $N$ be a given positive integer. Combining this fact with Proposition 5.3 we derive that, for every $j \geq N+1$,

$$
\left\|\triangle \varphi^{j}\right\|,\left\|\triangle \varphi_{ \pm}^{j}\right\|,\left\|\triangle \psi_{ \pm}^{j}\right\| \leq \Psi_{j-1} \leq 2^{-j+1+N} \Psi_{N} \ll 2^{-j+N}|x|^{-N+1}
$$

the implied constant not depending on $j$ but possibly on $N$. By Proposition 3.3 we conclude that

$$
\varphi^{\infty}=\sum_{j=2}^{\infty} \triangle \varphi^{j}+\varphi^{1}, \quad \varphi_{ \pm}^{\infty}=\sum_{j=2}^{\infty} \triangle \varphi_{ \pm}^{j}+\varphi_{ \pm}^{1}, \quad \psi_{ \pm}^{\infty}=\sum_{j=2}^{\infty} \triangle \psi_{ \pm}^{j}+\psi_{ \pm}^{1}
$$

belong to $\mathfrak{A}=\mathfrak{A}\left(B_{0}, B_{x}, B_{*}, \Sigma_{0}\left(x_{\infty}^{1}, \delta\right), \varepsilon\right)$ if $\varepsilon$ fulfils (5.4) with $r_{0}$ chosen as in Proposition 5.3. Thus we have constructed a solution $\left(\varphi, \varphi_{ \pm}, \psi_{ \pm}\right)=\left(\varphi^{\infty}, \varphi_{ \pm}^{\infty}, \psi_{ \pm}^{\infty}\right)$ of (5.1) and of (4.6). By (5.3), Propositions 3.3 and $5.5, \varphi^{\infty}, \varphi_{ \pm}^{\infty}, \psi_{ \pm}^{\infty}$ are written in the form

$$
\begin{aligned}
& \varphi^{\infty}=-\frac{1}{2}\left(\left(\sigma+\theta_{\infty}\right) \gamma_{+}^{0} \gamma_{-}^{0}+\left(\sigma-\theta_{\infty}\right) \gamma_{+}^{x} \gamma_{-}^{x}+\left[x^{-1}\right]\right) x^{-2} \\
& +\gamma_{-}^{0} \gamma_{+}^{x}(1)_{x} \mathrm{e}^{x} x^{\sigma-1}+\sum_{n=2}^{\infty}\left(\gamma_{-}^{0} \gamma_{+}^{x}\right)^{n}\left[x^{-n+1}\right]\left(\mathrm{e}^{x} x^{\sigma-1}\right)^{n} \\
& +\gamma_{+}^{0} \gamma_{-}^{x}(1)_{x} \mathrm{e}^{-x} x^{-\sigma-1}+\sum_{n=2}^{\infty}\left(\gamma_{+}^{0} \gamma_{-}^{x}\right)^{n}\left[x^{-n+1}\right]\left(\mathrm{e}^{-x} x^{-\sigma-1}\right)^{n}, \\
& \varphi_{+}^{\infty}=\gamma_{+}^{0}\left[x^{-1}\right]-\gamma_{+}^{x}\left(2 \gamma_{+}^{0} \gamma_{-}^{0}+\left[x^{-1}\right]\right) \mathrm{e}^{x} x^{\sigma-2}-\gamma_{-}^{0}\left(\gamma_{+}^{x}\right)^{2}(1)_{x}\left(\mathrm{e}^{x} x^{\sigma-1}\right)^{2} \\
& +\sum_{n=3}^{\infty} \gamma_{+}^{x}\left(\gamma_{-}^{0} \gamma_{+}^{x}\right)^{n-1}\left[x^{-n+2}\right]\left(\mathrm{e}^{x} x^{\sigma-1}\right)^{n}+2\left(\gamma_{+}^{0}\right)^{2} \gamma_{-}^{x}(1)_{x} \mathrm{e}^{-x} x^{-\sigma-2} \\
& +\sum_{n=2}^{\infty} \gamma_{+}^{0}\left(\gamma_{+}^{0} \gamma_{-}^{x}\right)^{n}\left[x^{-n}\right]\left(\mathrm{e}^{-x} x^{-\sigma-1}\right)^{n} \\
& \psi_{+}^{\infty}=\gamma_{+}^{x}\left[x^{-1}\right]+2 \gamma_{-}^{0}\left(\gamma_{+}^{x}\right)^{2}(1)_{x} \mathrm{e}^{x} x^{\sigma-2}+\sum_{n=2}^{\infty} \gamma_{+}^{x}\left(\gamma_{-}^{0} \gamma_{+}^{x}\right)^{n}\left[x^{-n}\right]\left(\mathrm{e}^{x} x^{\sigma-1}\right)^{n} \\
& -\gamma_{+}^{0}\left(2 \gamma_{+}^{x} \gamma_{-}^{x}+\left[x^{-1}\right]\right) \mathrm{e}^{-x} x^{-\sigma-2}-\left(\gamma_{+}^{0}\right)^{2} \gamma_{-}^{x}(1)_{x}\left(\mathrm{e}^{-x} x^{-\sigma-1}\right)^{2} \\
& +\sum_{n=3}^{\infty} \gamma_{+}^{0}\left(\gamma_{+}^{0} \gamma_{-}^{x}\right)^{n-1}\left[x^{-n+2}\right]\left(\mathrm{e}^{-x} x^{-\sigma-1}\right)^{n} \text {, } \\
& \varphi_{-}^{\infty}=\gamma_{-}^{0}\left[x^{-1}\right]+2\left(\gamma_{-}^{0}\right)^{2} \gamma_{+}^{x}(1)_{x} \mathrm{e}^{x} x^{\sigma-2}+\sum_{n=2}^{\infty} \gamma_{-}^{0}\left(\gamma_{-}^{0} \gamma_{+}^{x}\right)^{n}\left[x^{-n}\right]\left(\mathrm{e}^{x} x^{\sigma-1}\right)^{n} \\
& -\gamma_{-}^{x}\left(2 \gamma_{+}^{0} \gamma_{-}^{0}+\left[x^{-1}\right]\right) \mathrm{e}^{-x} x^{-\sigma-2}-\gamma_{+}^{0}\left(\gamma_{-}^{x}\right)^{2}(1)_{x}\left(\mathrm{e}^{-x} x^{-\sigma-1}\right)^{2} \\
& +\sum_{n=3}^{\infty} \gamma_{-}^{x}\left(\gamma_{+}^{0} \gamma_{-}^{x}\right)^{n-1}\left[x^{-n+2}\right]\left(\mathrm{e}^{-x} x^{-\sigma-1}\right)^{n}, \\
& \psi_{-}^{\infty}=\gamma_{-}^{x}\left[x^{-1}\right]-\gamma_{-}^{0}\left(2 \gamma_{+}^{x} \gamma_{-}^{x}+\left[x^{-1}\right]\right) \mathrm{e}^{x} x^{\sigma-2}-\left(\gamma_{-}^{0}\right)^{2} \gamma_{+}^{x}(1)_{x}\left(\mathrm{e}^{x} x^{\sigma-1}\right)^{2} \\
& +\sum_{n=3}^{\infty} \gamma_{-}^{0}\left(\gamma_{-}^{0} \gamma_{+}^{x}\right)^{n-1}\left[x^{-n+2}\right]\left(\mathrm{e}^{x} x^{\sigma-1}\right)^{n}+2 \gamma_{+}^{0}\left(\gamma_{-}^{x}\right)^{2}(1)_{x} \mathrm{e}^{-x} x^{-\sigma-2} \\
& +\sum_{n=2}^{\infty} \gamma_{-}^{x}\left(\gamma_{+}^{0} \gamma_{-}^{x}\right)^{n}\left[x^{-n}\right]\left(\mathrm{e}^{-x} x^{-\sigma-1}\right)^{n} .
\end{aligned}
$$


Then, by (4.8), $\Phi_{0}(x), \Phi_{x}(x) \rightarrow 0$ as $x \rightarrow \infty$ along a curve on which $\left|\mathrm{e}^{x} x^{\sigma}\right|=1$. Substitution of these into (4.7) leads us to the desired solution of Theorem 2.1.

Remark 5.6. By Remark 4.4, in the first equation of (5.1),

$$
\begin{gathered}
F_{0}\left(x, \varphi, \varphi_{+}, \psi_{+}, \varphi_{-}, \psi_{-}\right):=\mathrm{e}^{x} x^{\sigma-1}\left(1-(\sigma-1) x^{-1}+2 \kappa(x)\right)\left(\gamma_{-}^{0}+\varphi_{-}\right)\left(\gamma_{+}^{x}+\psi_{+}\right) \\
+\mathrm{e}^{-x} x^{-\sigma-1}\left(1-(\sigma+1) x^{-1}-2 \kappa(x)\right)\left(\gamma_{+}^{0}+\varphi_{+}\right)\left(\gamma_{-}^{x}+\psi_{-}\right)-\cdots .
\end{gathered}
$$

Using this fact, (4.7) and Proposition 5.4, and computing $\varphi^{2}, \varphi_{ \pm}^{2}, \psi_{ \pm}^{2}$, we may write some terms of the expressions for $f_{0}, f_{ \pm}, g_{ \pm}$in Theorem 2.1 in more detail:

$$
\begin{aligned}
& f_{0}=\cdots+\gamma_{-}^{0} \gamma_{+}^{x}\left(1-\left(\sigma-1+2\left(\gamma_{+}^{0} \gamma_{-}^{0}+\gamma_{+}^{x} \gamma_{-}^{x}\right)-\left(\sigma^{2}-\theta_{\infty}^{2}\right) / 2\right) x^{-1}+\left[x^{-2}\right]\right) \mathrm{e}^{x} x^{\sigma-1} \\
& \quad+\gamma_{+}^{0} \gamma_{-}^{x}\left(1-\left(\sigma+1-2\left(\gamma_{+}^{0} \gamma_{-}^{0}+\gamma_{+}^{x} \gamma_{-}^{x}\right)+\left(\sigma^{2}-\theta_{\infty}^{2}\right) / 2\right) x^{-1}+\left[x^{-2}\right]\right) \mathrm{e}^{-x} x^{-\sigma-1}+\cdots, \\
& x^{\left(\sigma+\theta_{\infty}\right) / 2} f_{+}=\gamma_{+}^{0}\left(1+\left(2 \gamma_{+}^{x} \gamma_{-}^{x}-\left(\sigma^{2}-\theta_{\infty}^{2}\right) / 4\right) x^{-1}+\left[x^{-2}\right]\right)+\cdots, \\
& \mathrm{e}^{-x} x^{-\left(\sigma-\theta_{\infty}\right) / 2} g_{+}=\gamma_{+}^{x}\left(1-\left(2 \gamma_{+}^{0} \gamma_{-}^{0}-\left(\sigma^{2}-\theta_{\infty}^{2}\right) / 4\right) x^{-1}+\left[x^{-2}\right]\right)+\cdots, \\
& x^{-\left(\sigma+\theta_{\infty}\right) / 2} f_{-}=\gamma_{-}^{0}\left(1-\left(2 \gamma_{+}^{x} \gamma_{-}^{x}-\left(\sigma^{2}-\theta_{\infty}^{2}\right) / 4\right) x^{-1}+\left[x^{-2}\right]\right)+\cdots, \\
& \mathrm{e}^{x} x^{\left(\sigma-\theta_{\infty}\right) / 2} g_{-}=\gamma_{-}^{x}\left(1+\left(2 \gamma_{+}^{0} \gamma_{-}^{0}-\left(\sigma^{2}-\theta_{\infty}^{2}\right) / 4\right) x^{-1}+\left[x^{-2}\right]\right)+\cdots .
\end{aligned}
$$

These facts are used in computing the tau-function.

\subsection{Proof of Theorem 2.8}

In the proof of Theorem 2.1 described above, we put $\sigma=\sigma_{0}=-2 \theta_{x}-\theta_{\infty}$, namely $\gamma_{-}^{x}=0$, to obtain the solution of Theorem 2.8 in the sector $|\arg x-\pi / 2|<\pi / 2-\delta,\left|\mathrm{e}^{x} x^{\sigma_{0}-1}\right|<\varepsilon$, since the restriction $\left|\mathrm{e}^{-x} x^{-\sigma_{0}-1}\right|<\varepsilon$ is removed. It is sufficient to show that this expression may be extended to the sector $|\arg x-\pi|<\pi / 2-\delta$. In the sector $|\arg x-\pi / 2|<\pi / 2-\delta$, write $\left(\varphi^{\infty}, \varphi_{ \pm}^{\infty}, \psi_{ \pm}^{\infty}\right)$ with $\sigma=\sigma_{0}$ in the form

$$
\begin{array}{llrl}
\varphi^{\infty} & =p(x)+\mathrm{e}^{x} x^{\sigma_{0}} \hat{\varphi}^{\infty}, & \varphi_{ \pm}^{\infty} & =p_{ \pm}(x)+\mathrm{e}^{x} x^{\sigma_{0}} \hat{\varphi}_{ \pm}^{\infty}, \\
\psi_{+}^{\infty}=q_{+}(x) \mathrm{e}^{-x} x^{-\sigma_{0}}+\hat{\psi}_{+}^{\infty}, & \psi_{-}^{\infty}=q_{-}(x) \mathrm{e}^{x} x^{\sigma_{0}}+\left(\mathrm{e}^{x} x^{\sigma_{0}}\right)^{2} \hat{\psi}_{-}^{\infty},
\end{array}
$$

where $p(x)=\left[x^{-2}\right], p_{ \pm}(x)=\left[x^{-1}\right], q_{ \pm}(x)=\left[x^{-3}\right]$, and $\hat{\varphi}^{\infty}, \hat{\varphi}_{ \pm}^{\infty}, \hat{\psi}_{ \pm}^{\infty} \in \mathfrak{A}\left(\Sigma_{0}\left(x_{\infty}^{1}, \delta\right), \varepsilon\right)$ $\left(=\mathfrak{A}\left(B_{0}, B_{x},\left\{\sigma_{0}\right\}, \Sigma_{0}\left(x_{\infty}^{1}, \delta\right), \varepsilon\right)\right)$. Note that $\hat{\varphi}^{\infty}, \hat{\varphi}_{ \pm}^{\infty}, \hat{\psi}_{ \pm}^{\infty}$ also belong to $\mathfrak{A}_{+}\left(\Sigma_{\pi}(\pi / 2+\delta, \pi-\right.$ $\left.\left.\delta ; x_{\infty}^{1}\right), \varepsilon\right)$. Recall that $\left(\varphi^{\infty}, \varphi_{ \pm}^{\infty}, \psi_{ \pm}^{\infty}\right)$ solves (5.1) with $\gamma_{-}^{x}=0$. Inserting (5.15) into this system and putting $\gamma_{+}^{x}=0$, we find that $\left(p(x), p_{ \pm}(x), q_{ \pm}(x)\right)$ solves (5.1) with $\gamma_{-}^{x}=\gamma_{+}^{x}=0$, namely (5.1) with

$$
\begin{aligned}
F_{0}= & F_{0}^{*}\left(x, \varphi, \varphi_{+}, \psi_{+}, \varphi_{-}, \psi_{-}\right)=x^{-1}\left((1)_{x}\left(\gamma_{-}^{0}+\varphi_{-}\right) \psi_{+}+(1)_{x}\left(\gamma_{+}^{0}+\varphi_{+}\right) \psi_{-}\right) \\
& -4 \mathcal{I}\left[x^{-2} \varphi\left((1)_{x}\left(\gamma_{-}^{0}+\varphi_{-}\right) \psi_{+}-(1)_{x}\left(\gamma_{+}^{0}+\varphi_{+}\right) \psi_{-}\right)\right] \\
& +\mathcal{I}\left[x^{-3}\left(([1]+[1] \varphi)\left(\gamma_{+}^{0}+\varphi_{+}\right)\left(\gamma_{-}^{0}+\varphi_{-}\right)+([1]+[1] \varphi) \psi_{+} \psi_{-}\right)\right], \\
F_{+}= & F_{+}^{*}\left(x, \varphi, \varphi_{+}, \psi_{+}\right)=-2 \mathcal{I}\left[x^{-1} \varphi\left((1)_{x}\left(\gamma_{+}^{0}+\varphi_{+}\right)+(1)_{x} \psi_{+}\right)\right], \\
G_{+}= & G_{+}^{*}\left(x, \varphi, \varphi_{+}, \psi_{+}\right)=2 \mathrm{e}^{x} x^{\sigma_{0}} \mathcal{I}\left[\mathrm{e}^{-x} x^{-\sigma_{0}-1} \varphi\left((1)_{x}\left(\gamma_{+}^{0}+\varphi_{+}\right)+(1)_{x} \psi_{+}\right)\right], \\
F_{-}= & F_{-}^{*}\left(x, \varphi, \varphi_{-}, \psi_{-}\right)=2 \mathcal{I}\left[x^{-1} \varphi\left((1)_{x}\left(\gamma_{-}^{0}+\varphi_{-}\right)+(1)_{x} \psi_{-}\right)\right], \\
G_{-}= & G_{-}^{*}\left(x, \varphi, \varphi_{-}, \psi_{-}\right)=-2 \mathrm{e}^{-x} x^{-\sigma_{0}} \mathcal{I}\left[\mathrm{e}^{x} x^{\sigma_{0}-1} \varphi\left((1)_{x}\left(\gamma_{-}^{0}+\varphi_{-}\right)+(1)_{x} \psi_{-}\right)\right] .
\end{aligned}
$$

Let $\left(\varphi^{j}, \varphi_{ \pm}^{j}, \psi_{ \pm}^{j}\right)$ be the sequence defined by

$$
\begin{aligned}
& \varphi^{0}=\varphi_{ \pm}^{0}=\psi_{ \pm}^{0} \equiv 0, \\
& \varphi^{j+1}=F^{*}\left(x, \varphi^{j}, \varphi_{+}^{j}, \psi_{+}^{j}, \varphi_{-}^{j}, \psi_{-}^{j}\right), \\
& \varphi_{ \pm}^{j+1}=F_{ \pm}^{*}\left(x, \varphi^{j+1}, \varphi_{ \pm}^{j}, \psi_{ \pm}^{j}\right), \quad \psi_{ \pm}^{j+1}=G_{ \pm}^{*}\left(x, \varphi^{j+1}, \varphi_{ \pm}^{j}, \psi_{ \pm}^{j}\right) .
\end{aligned}
$$


Here, for $|\arg x-\pi / 2|<\pi-\delta$, we may replace the path $\gamma(x)$ by a ray tending to $\infty \mathrm{e}^{\mathrm{i} \vartheta}$ such that $|\vartheta|<\pi / 2-\delta$ (respectively, $|\vartheta-\pi|<\pi / 2-\delta$ ) in $G_{+}^{*}$ (respectively, $G_{-}^{*}$ ) and by one tending to $\infty \mathrm{e}^{\mathrm{i} \arg x}$ in the others. Then the sequence converges to $\left(p^{\infty}(x), p_{ \pm}^{\infty}(x), q_{ \pm}^{\infty}(x)\right)$ whose entries admit asymptotic expansions in the sector $|\arg x-\pi / 2|<\pi-\delta$. Since (5.1) with $\gamma_{-}^{x}=\gamma_{+}^{x}=0$ whose paths are replaced as above has a unique solution tending to 0 in this sector, the asymptotic expression for $\left(p(x), p_{ \pm}(x), q_{ \pm}(x)\right)$ is valid in the sector $|\arg x-\pi / 2|<\pi-\delta$. By the fact that $\left(p(x), p_{ \pm}(x), q_{ \pm}(x)\right)$ solves this system, $\left(\hat{\varphi}, \hat{\varphi}_{ \pm}, \hat{\psi}_{ \pm}\right)=\left(\hat{\varphi}^{\infty}, \hat{\varphi}_{ \pm}^{\infty}, \hat{\psi}_{ \pm}^{\infty}\right)$ satisfies a system of the form

$$
\begin{aligned}
\hat{\varphi}= & {\left[x^{-1}\right]+x^{-1}\left(\left[x^{-2}\right] \hat{\varphi}_{+}+(*) \hat{\varphi}_{-}+[1] \hat{\psi}_{+}+[1] \hat{\psi}_{-}+(*) \hat{\varphi}_{-} \hat{\psi}_{+}+(*) \hat{\varphi}_{+} \hat{\psi}_{-}\right) } \\
& +\left(\mathrm{e}^{x} x^{\sigma_{0}}\right)^{-1} \mathcal{I}\left[\mathrm { e } ^ { x } x ^ { \sigma _ { 0 } - 1 } \left((*) \hat{\varphi}+\left[x^{-1}\right] \hat{\varphi}_{+}+(*) \hat{\varphi}_{-}+\left[x^{-1}\right] \hat{\psi}_{+}+(*) \hat{\psi}_{-}\right.\right. \\
& +(*) \hat{\varphi}_{+} \hat{\varphi}_{-}+(*) \hat{\psi}_{+} \hat{\psi}_{-}+(*) \hat{\varphi}_{-} \hat{\psi}_{+}+(*) \hat{\varphi}_{+} \hat{\psi}_{-}+\hat{\varphi}\left((*) \hat{\varphi}_{+}+(*) \hat{\varphi}_{-}\right. \\
& \left.\left.\left.+(*) \hat{\psi}_{+}+(*) \hat{\psi}_{-}+(*) \hat{\varphi}_{+} \hat{\varphi}_{-}+(*) \hat{\psi}_{+} \hat{\psi}_{-}+(*) \hat{\varphi}_{-} \hat{\psi}_{+}+(*) \hat{\varphi}_{+} \hat{\psi}_{-}\right)\right)\right], \\
\hat{\varphi}_{+}= & {\left[x^{-1}\right]+\left(\mathrm{e}^{x} x^{\sigma_{0}}\right)^{-1} } \\
& \times \mathcal{I}\left[\mathrm{e}^{x} x^{\sigma_{0}-1}\left(([1]+(*)) \hat{\varphi}+\left[x^{-2}\right] \hat{\varphi}_{+}+\left[x^{-2}\right] \hat{\psi}_{+}+\hat{\varphi}\left((*) \hat{\varphi}_{+}+(*) \hat{\psi}_{+}\right)\right)\right], \\
\hat{\psi}_{+}= & {\left[x^{-1}\right]+\mathcal{I}\left[x^{-1}\left(([1]+(*)) \hat{\varphi}+\left[x^{-2}\right] \hat{\varphi}_{+}+\left[x^{-2}\right] \hat{\psi}_{+}+\hat{\varphi}\left((*) \hat{\varphi}_{+}+(*) \hat{\psi}_{+}\right)\right)\right], } \\
\hat{\varphi}_{-}= & \left(\mathrm{e}^{x} x^{\sigma_{0}}\right)^{-1} \mathcal{I}\left[\mathrm{e}^{x} x^{\sigma_{0}-1}\left([1] \hat{\varphi}+\left[x^{-2}\right] \hat{\varphi}_{-}+\left[x^{-2}\right] \hat{\psi}_{-}+\hat{\varphi}\left((*) \hat{\varphi}_{-}+(*) \hat{\psi}_{-}\right)\right)\right], \\
\hat{\psi}_{-}= & \left(\mathrm{e}^{x} x^{\sigma_{0}}\right)^{-2} \mathcal{I}\left[\left(\mathrm{e}^{x} x^{\sigma_{0}}\right)^{2} x^{-1}\left([1] \hat{\varphi}+\left[x^{-2}\right] \hat{\varphi}_{-}+\left[x^{-2}\right] \hat{\psi}_{-}+\hat{\varphi}\left((*) \hat{\varphi}_{-}+(*) \hat{\psi}_{-}\right)\right)\right],
\end{aligned}
$$

where every asymptotic coefficient is valid in the sector $|\arg x-\pi / 2|<\pi-\delta$, and each (*) denotes a function of the form $\left[x^{-1}\right]+[1] \mathrm{e}^{x} x^{\sigma_{0}}+[1]\left(\mathrm{e}^{x} x^{\sigma_{0}}\right)^{2}$. For $|\arg x-\pi|<\pi / 2-\delta$, replace $\gamma(x)$ by $\gamma_{\pi}(x)$ (cf. Section 3.2), and define the sequence $\left(\hat{\varphi}^{j}, \hat{\varphi}_{ \pm}^{j}, \hat{\psi}_{ \pm}^{j}\right)$ by the same way as in (5.2). Then, using the facts in Section 3.2, we may construct a solution $\left(\hat{\varphi}^{*}, \hat{\varphi}_{ \pm}^{*}, \hat{\psi}_{ \pm}^{*}\right)$ whose entries are in $\mathfrak{A}_{+}\left(\Sigma_{\pi}\left(\pi / 2+\delta, 3 \pi / 2-\delta, x_{\infty}^{1}\right), \varepsilon\right)$. This coincides with $\left(\hat{\varphi}^{\infty}, \hat{\varphi}_{ \pm}^{\infty}, \hat{\psi}_{ \pm}^{\infty}\right)$ in the sector $\pi / 2+\delta<\arg x<\pi-\delta$, since the corresponding asymptotic coefficients of these solutions satisfy the same recursive relation. This completes the proof of Theorem 2.8.

\section{Proofs of the results on (V)}

Let $\left(f_{0}, f_{ \pm}, g_{ \pm}\right)$be the solution given by Theorem 2.1 or 2.8 , which has been obtained by constructing $\left(\varphi^{\infty}, \varphi_{ \pm}^{\infty}, \psi_{ \pm}^{\infty}\right)$ that solves (5.1) in Section 2.3. Then, by (1.2),

$$
y=\frac{g_{+}\left(f_{0}+\theta_{0} / 2\right)}{f_{+}\left(g_{0}+\theta_{x} / 2\right)}, \quad g_{0}=-f_{0}-\theta_{\infty} / 2
$$

is a solution of $(\mathrm{V})$.

\subsection{Proofs of Theorems 2.18 and 2.21}

We begin with the following:

Proposition 6.1. The solution $y$ depends on the parameters $\sigma$ and $c=c_{x} / c_{0}$ (respectively, $\left.c^{\prime}=c_{0} / c_{x}\right)$ only.

Proof. Note that the coefficients of each asymptotic series $\left[x^{-1}\right]$ in $(5.1)$ are in $\mathbb{Q}\left[\theta_{0}, \theta_{x}, \theta_{\infty}, \sigma\right]$. We may suppose that $\gamma_{ \pm}^{0}, \gamma_{ \pm}^{x} \neq 0$. Set $\varphi_{+}=\gamma_{+}^{0} \tilde{\varphi}_{+}, \psi_{+}=\gamma_{+}^{x} \tilde{\psi}_{+}, \varphi_{-}=\gamma_{-}^{0} \tilde{\varphi}_{-}, \psi_{-}=\gamma_{-}^{x} \tilde{\psi}_{-}$. Then (5.1) becomes

$$
\varphi=\gamma_{-}^{0} \gamma_{+}^{x}(1)_{x} \mathrm{e}^{x} x^{\sigma-1}\left(1+\tilde{\varphi}_{-}\right)\left(1+\tilde{\psi}_{+}\right)+\gamma_{+}^{0} \gamma_{-}^{x}(1)_{x} \mathrm{e}^{-x} x^{-\sigma-1}\left(1+\tilde{\varphi}_{+}\right)\left(1+\tilde{\psi}_{-}\right)
$$




$$
\begin{aligned}
& -4 \mathcal{I}\left[\gamma_{-}^{0} \gamma_{+}^{x}(1)_{x} \mathrm{e}^{x} x^{\sigma-2} \varphi\left(1+\tilde{\varphi}_{-}\right)\left(1+\tilde{\psi}_{+}\right)-\gamma_{+}^{0} \gamma_{-}^{x}(1)_{x} \mathrm{e}^{-x} x^{-\sigma-2} \varphi\left(1+\tilde{\varphi}_{+}\right)\left(1+\tilde{\psi}_{-}\right)\right] \\
& +\mathcal{I}\left[\gamma_{+}^{0} \gamma_{-}^{0} x^{-3}([1]+[1] \varphi)\left(1+\tilde{\varphi}_{+}\right)\left(1+\tilde{\varphi}_{-}\right)+\gamma_{+}^{x} \gamma_{-}^{x} x^{-3}([1]+[1] \varphi)\left(1+\tilde{\psi}_{+}\right)\left(1+\tilde{\psi}_{-}\right)\right], \\
\tilde{\varphi}_{+}= & -2 \mathcal{I}\left[\varphi\left(x^{-1}(1)_{x}\left(1+\tilde{\varphi}_{+}\right)+\left(\gamma_{+}^{x} / \gamma_{+}^{0}\right) \mathrm{e}^{x} x^{\sigma-1}(1)_{x}\left(1+\tilde{\psi}_{+}\right)\right)\right], \\
\tilde{\psi}_{+}= & 2 \mathcal{I}\left[\varphi\left(x^{-1}(1)_{x}\left(1+\tilde{\psi}_{+}\right)+\left(\gamma_{+}^{0} / \gamma_{+}^{x}\right) \mathrm{e}^{-x} x^{-\sigma-1}(1)_{x}\left(1+\tilde{\varphi}_{+}\right)\right)\right], \\
\tilde{\varphi}_{-}= & 2 \mathcal{I}\left[\varphi\left(x^{-1}(1)_{x}\left(1+\tilde{\varphi}_{-}\right)+\left(\gamma_{-}^{x} / \gamma_{-}^{0}\right) \mathrm{e}^{-x} x^{-\sigma-1}(1)_{x}\left(1+\tilde{\psi}_{-}\right)\right)\right], \\
\tilde{\psi}_{-}= & -2 \mathcal{I}\left[\varphi\left(x^{-1}(1)_{x}\left(1+\tilde{\psi}_{-}\right)+\left(\gamma_{-}^{0} / \gamma_{-}^{x}\right) \mathrm{e}^{x} x^{\sigma-1}(1)_{x}\left(1+\tilde{\varphi}_{-}\right)\right)\right] .
\end{aligned}
$$

This implies that, for $\left(\varphi^{\infty}, \varphi_{ \pm}^{\infty}, \psi_{ \pm}^{\infty}\right)$, the corresponding solution $\left(\tilde{\varphi}^{\infty}, \tilde{\varphi}_{ \pm}^{\infty}, \tilde{\psi}_{ \pm}^{\infty}\right)$ of $(6.2)$ depends on $\sigma$ and $c=c_{x} / c_{0}$ only, since $\gamma_{-}^{0} \gamma_{+}^{x}, \gamma_{+}^{0} \gamma_{-}^{x}, \gamma_{+}^{x} / \gamma_{+}^{0}, \gamma_{-}^{x} / \gamma_{-}^{0}$ (respectively, $\gamma_{+}^{0} \gamma_{-}^{0}, \gamma_{+}^{x} \gamma_{-}^{x}$ ) are written in terms of $\sigma$ and $c$ (respectively, $\sigma$ ) only. From (4.7) it follows that

$$
\begin{aligned}
& f_{0}=-g_{0}-\theta_{\infty} / 2=\left(\sigma-\theta_{\infty}\right) / 4+\varphi^{\infty} \\
& \frac{g_{+}}{f_{+}}=\frac{(1)_{x} c \mathrm{e}^{x} x^{\sigma}\left(1+\tilde{\psi}_{+}^{\infty}\right)-\left(\left(\sigma+\theta_{\infty}\right) / 2+\left[x^{-1}\right]\right) x^{-1}\left(1+\tilde{\varphi}_{+}^{\infty}\right)}{(1)_{x}\left(1+\tilde{\varphi}_{+}^{\infty}\right)-\left(\left(\sigma-\theta_{\infty}\right) / 2+\left[x^{-1}\right]\right) c \mathrm{e}^{x} x^{\sigma-1}\left(1+\tilde{\psi}_{+}^{\infty}\right)},
\end{aligned}
$$

and hence the proposition follows immediately.

Suppose $\operatorname{dist}\left(\left\{-2 \theta_{0}+\theta_{\infty}, 2 \theta_{x}-\theta_{\infty}\right\}, B_{*}\right)=d_{0}>0$, that is, $\left|\gamma_{+}^{0} / c_{0}\right|,\left|\gamma_{+}^{x} / c_{x}\right| \geq d_{0} / 4$ for every $\sigma \in B_{*}$. Then, by Theorem 2.1 combined with the expression of $\varphi^{\infty}$ in Section 5.4,

$$
\begin{aligned}
& \frac{f_{0}+\theta_{0} / 2}{g_{0}+\theta_{x} / 2}=\frac{\gamma_{+}^{0} / c_{0}+\varphi^{\infty}}{\gamma_{+}^{x} / c_{x}-\varphi^{\infty}}=\left(1+\frac{c_{x} \gamma_{+}^{0}}{c_{0} \gamma_{+}^{x}}\right)\left(1-\frac{c_{x}}{\gamma_{+}^{x}} \varphi^{\infty}\right)^{-1}-1, \\
& x^{\left(\sigma+\theta_{\infty}\right) / 2} g_{+}=\gamma_{+}^{x} \mathrm{e}^{x} x^{\sigma}(1)_{x}\left(1+\left(\gamma_{+}^{x}\right)^{-1} \hat{g}_{+}\right), \\
& \left(x^{\left(\sigma+\theta_{\infty}\right) / 2} f_{+}\right)^{-1}=\left(\gamma_{+}^{0}\right)^{-1}(1)_{x}\left(1-\left(\gamma_{+}^{0}\right)^{-1} \hat{f}_{+}\right)^{-1},
\end{aligned}
$$

provided that $\left|\mathrm{e}^{x} x^{\sigma-1}\right|$ and $\left|\mathrm{e}^{-x} x^{-\sigma-1}\right|$ are sufficiently small, where

$$
\begin{aligned}
\hat{g}_{+}= & \sum_{n=1}^{\infty} \gamma_{+}^{x}\left(\gamma_{-}^{0} \gamma_{+}^{x}\right)^{n}\left[x^{-n}\right]\left(\mathrm{e}^{x} x^{\sigma-1}\right)^{n}-\gamma_{+}^{0}\left(\left(\sigma+\theta_{\infty}\right) / 2+\left[x^{-1}\right]\right) \mathrm{e}^{-x} x^{-\sigma-1} \\
& -\left(\gamma_{+}^{0}\right)^{2} \gamma_{-}^{x}(1)_{x}\left(\mathrm{e}^{-x} x^{-\sigma-1}\right)^{2}+\sum_{n=3}^{\infty} \gamma_{+}^{0}\left(\gamma_{+}^{0} \gamma_{-}^{x}\right)^{n-1}\left[x^{-n+2}\right]\left(\mathrm{e}^{-x} x^{-\sigma-1}\right)^{n}, \\
\hat{f}_{+}= & \gamma_{+}^{x}\left(\left(\sigma-\theta_{\infty}\right) / 2+\left[x^{-1}\right]\right) \mathrm{e}^{x} x^{\sigma-1}+\gamma_{-}^{0}\left(\gamma_{+}^{x}\right)^{2}(1)_{x}\left(\mathrm{e}^{x} x^{\sigma-1}\right)^{2} \\
& +\sum_{n=3}^{\infty} \gamma_{+}^{x}\left(\gamma_{-}^{0} \gamma_{+}^{x}\right)^{n-1}\left[x^{-n+2}\right]\left(\mathrm{e}^{x} x^{\sigma-1}\right)^{n}+\sum_{n=1}^{\infty} \gamma_{+}^{0}\left(\gamma_{+}^{0} \gamma_{-}^{x}\right)^{n}\left[x^{-n}\right]\left(\mathrm{e}^{-x} x^{-\sigma-1}\right)^{n} .
\end{aligned}
$$

Furthermore

$$
\begin{aligned}
& \left(1-c_{x}\left(\gamma_{+}^{x}\right)^{-1} \varphi^{\infty}\right)^{-1}=\left(1-\chi_{0}\right)^{-1}\left(1-x^{-1} \varphi_{*}^{\infty}\left(1-\chi_{0}\right)^{-1}\right)^{-1}, \\
& \left(1-\left(\gamma_{+}^{0}\right)^{-1} \hat{f}_{+}\right)^{-1}=\left(1-\chi_{1}\right)^{-1}\left(1-x^{-1} \hat{f}_{+*}\left(1-\chi_{1}\right)^{-1}\right)^{-1},
\end{aligned}
$$

where

$$
\begin{aligned}
& \chi_{0}=c_{x}\left(\gamma_{+}^{x}\right)^{-1}\left(\gamma_{-}^{0} \gamma_{+}^{x}(1)_{x} \mathrm{e}^{x} x^{\sigma-1}+\gamma_{+}^{0} \gamma_{-}^{x}(1)_{x} \mathrm{e}^{-x} x^{-\sigma-1}\right), \\
& x^{-1} \varphi_{*}^{\infty}=c_{x}\left(\gamma_{+}^{x}\right)^{-1}\left(\varphi^{\infty}-\gamma_{-}^{0} \gamma_{+}^{x}(1)_{x} \mathrm{e}^{x} x^{\sigma-1}-\gamma_{+}^{0} \gamma_{-}^{x}(1)_{x} \mathrm{e}^{-x} x^{-\sigma-1}\right), \\
& \chi_{1}=\left(\gamma_{+}^{0}\right)^{-1}\left(\gamma_{+}^{x}\left(\left(\sigma-\theta_{\infty}\right) / 2+\left[x^{-1}\right]\right) \mathrm{e}^{x} x^{\sigma-1}+\gamma_{-}^{0}\left(\gamma_{+}^{x}\right)^{2}(1)_{x}\left(\mathrm{e}^{x} x^{\sigma-1}\right)^{2}\right), \\
& x^{-1} \hat{f}_{+*}=\left(\gamma_{+}^{0}\right)^{-1}\left(\hat{f}_{+}-\gamma_{+}^{x}\left(\left(\sigma-\theta_{\infty}\right) / 2+\left[x^{-1}\right]\right) \mathrm{e}^{x} x^{\sigma-1}-\gamma_{-}^{0}\left(\gamma_{+}^{x}\right)^{2}(1)_{x}\left(\mathrm{e}^{x} x^{\sigma-1}\right)^{2}\right) .
\end{aligned}
$$


By Proposition 3.3 with Examples 3.5 and 3.6, substitution of these expressions into (6.1) yields $y(c, \sigma, x)$ as in Theorem 2.18.

If we set $\sigma=\sigma_{0}=-2 \theta_{x}-\theta_{\infty}$, that is, $\gamma_{-}^{x}=0$, then the coefficients of the solution in Theorem 2.18 are such that $b_{n}=0$ for $n \geq 2$, and we obtain the solution $y_{+}(c, x)$. If $\sigma=\sigma_{0}^{\prime}=2 \theta_{0}+\theta_{\infty}$, that is, $\gamma_{-}^{0}=0$, then

$$
\begin{aligned}
& x^{\left(\sigma_{0}^{\prime}+\theta_{\infty}\right) / 2} f_{+}=\gamma_{+}^{0}(1)_{x}\left(1-\left(\gamma_{+}^{0}\right)^{-1} \hat{f}_{+}\right), \\
& \left(x^{\left(\sigma_{0}^{\prime}+\theta_{\infty}\right) / 2} g_{+}\right)^{-1}=\left(\gamma_{+}^{x}\right)^{-1} \mathrm{e}^{-x} x^{-\sigma_{0}^{\prime}}(1)_{x}\left(1+\left(\gamma_{+}^{x}\right)^{-1} \hat{g}_{+}\right)^{-1},
\end{aligned}
$$

from which the solution $y_{-}\left(c^{\prime}, x\right)$ follows. Thus Theorem 2.21 is obtained.

\subsection{Proofs of Theorems 2.26 and 2.27}

To discuss the poles and zeros of $y(c, \sigma, x)$, under the condition $\gamma_{ \pm}^{0}, \gamma_{ \pm}^{x} \neq 0$ we write

$$
\begin{gathered}
\mathrm{e}^{-x} x^{-\left(\sigma-\theta_{\infty}\right) / 2} g_{+}=\gamma_{+}^{x}-\left(\gamma_{+}^{0} / 2\right)\left(\sigma+\theta_{\infty}\right) \mathrm{e}^{-x} x^{-\sigma-1}-\left(\gamma_{+}^{0}\right)^{2} \gamma_{-}^{x}\left(\mathrm{e}^{-x} x^{-\sigma-1}\right)^{2}+O\left(x^{-1}\right) \\
=-\left(\gamma_{+}^{0}\right)^{2} \gamma_{-}^{x}\left(\mathrm{e}^{-x} x^{-\sigma-1}-\varrho_{1}\right)\left(\mathrm{e}^{-x} x^{-\sigma-1}-\varrho_{2}\right)+O\left(x^{-1}\right), \\
x^{\left(\sigma+\theta_{\infty}\right) / 2} f_{+}=\gamma_{+}^{0}-\left(\gamma_{+}^{x} / 2\right)\left(\sigma-\theta_{\infty}\right) \mathrm{e}^{x} x^{\sigma-1}-\gamma_{-}^{0}\left(\gamma_{+}^{x}\right)^{2}\left(\mathrm{e}^{x} x^{\sigma-1}\right)^{2}+O\left(x^{-1}\right) \\
=-\gamma_{-}^{0}\left(\gamma_{+}^{x}\right)^{2}\left(\mathrm{e}^{x} x^{\sigma-1}-\tilde{\varrho}_{1}\right)\left(\mathrm{e}^{x} x^{\sigma-1}-\tilde{\varrho}_{2}\right)+O\left(x^{-1}\right)
\end{gathered}
$$

with

$$
\begin{array}{ll}
\varrho_{1}=-\frac{c_{x}}{\gamma_{+}^{0}}=\frac{-4 c}{\sigma+2 \theta_{0}-\theta_{\infty}}, & \varrho_{2}=\frac{\gamma_{+}^{x}}{c_{x} \gamma_{+}^{0} \gamma_{-}^{x}}=\frac{-4 c\left(\sigma-2 \theta_{x}+\theta_{\infty}\right)}{\left(\sigma+2 \theta_{x}+\theta_{\infty}\right)\left(\sigma+2 \theta_{0}-\theta_{\infty}\right)}, \\
\tilde{\varrho}_{1}=\frac{c_{0}}{\gamma_{+}^{x}}=\frac{-4}{c\left(\sigma-2 \theta_{x}+\theta_{\infty}\right)}, & \tilde{\varrho}_{2}=-\frac{\gamma_{+}^{0}}{c_{0} \gamma_{-}^{0} \gamma_{+}^{x}}=\frac{-4\left(\sigma+2 \theta_{0}-\theta_{\infty}\right)}{c\left(\sigma-2 \theta_{0}-\theta_{\infty}\right)\left(\sigma-2 \theta_{x}+\theta_{\infty}\right)},
\end{array}
$$

provided that $\left|\mathrm{e}^{x} x^{\sigma-1}\right|,\left|\mathrm{e}^{-x} x^{-\sigma-1}\right|<\varepsilon$. Furthermore, for $\varepsilon^{-1}|x|^{-1}<\left|\mathrm{e}^{-x} x^{-\sigma-1}\right|<\varepsilon,\left|\mathrm{e}^{x} x^{\sigma-1}\right|$ $<\varepsilon|x|^{-1}$,

$$
\begin{aligned}
& f_{0}+\theta_{0} / 2=\gamma_{+}^{0} \gamma_{-}^{x}\left(\mathrm{e}^{-x} x^{-\sigma-1}-\varrho_{3}\right)+O\left(x^{-1}\right), \\
& g_{0}+\theta_{x} / 2=-\gamma_{+}^{0} \gamma_{-}^{x}\left(\mathrm{e}^{-x} x^{-\sigma-1}-\varrho_{2}\right)+O\left(x^{-1}\right),
\end{aligned}
$$

and for $\varepsilon^{-1}|x|^{-1}<\left|\mathrm{e}^{x} x^{\sigma-1}\right|<\varepsilon,\left|\mathrm{e}^{-x} x^{-\sigma-1}\right|<\varepsilon|x|^{-1}$,

$$
\begin{aligned}
& f_{0}+\theta_{0} / 2=\gamma_{-}^{0} \gamma_{+}^{x}\left(\mathrm{e}^{x} x^{\sigma-1}-\tilde{\varrho}_{2}\right)+O\left(x^{-1}\right), \\
& g_{0}+\theta_{x} / 2=-\gamma_{-}^{0} \gamma_{+}^{x}\left(\mathrm{e}^{x} x^{\sigma-1}-\tilde{\varrho}_{3}\right)+O\left(x^{-1}\right),
\end{aligned}
$$

where

$$
\varrho_{3}=-\frac{1}{c_{0} \gamma_{-}^{x}}=\frac{-4 c}{\sigma+2 \theta_{x}+\theta_{\infty}}, \quad \tilde{\varrho}_{3}=\frac{1}{c_{x} \gamma_{-}^{0}}=\frac{-4}{c\left(\sigma-2 \theta_{0}-\theta_{\infty}\right)} .
$$

If $\theta_{x}\left(\theta_{0} \pm \theta_{x}-\theta_{\infty}\right) \neq 0$ (respectively, $\theta_{0}\left( \pm \theta_{0}-\theta_{x}+\theta_{\infty}\right) \neq 0$ ), $\varrho_{1}, \varrho_{2}, \varrho_{3}$ (respectively, $\varrho_{1}, \tilde{\varrho}_{2}$, $\left.\tilde{\varrho}_{3}\right)$ are distinct. By the expressions above, in the domain $\varepsilon^{-1}|x|^{-1}<\left|\mathrm{e}^{-x} x^{-\sigma-1}\right|<\varepsilon$, where $\varepsilon$ is a positive number such that

$$
\begin{gathered}
\left(\left|\gamma_{-}^{0} \gamma_{+}^{x}\right|+\left|\gamma_{+}^{0} \gamma_{-}^{x}\right|+\left|\gamma_{+}^{0}\right|+\left|\gamma_{-}^{0}\right|+\left|\gamma_{+}^{x}\right|+\left|\gamma_{-}^{x}\right|+1\right) \\
\times\left(\left|\gamma_{+}^{0}\right|+\left|\gamma_{-}^{0}\right|+\left|\gamma_{+}^{x}\right|+\left|\gamma_{-}^{x}\right|+1\right) \varepsilon \leq r_{0}(\delta)
\end{gathered}
$$

(cf. Remark 2.3), $y(c, \sigma, x)$ admits a zero $x^{(0)}$ such that $\mathrm{e}^{-x^{(0)}}\left(x^{(0)}\right)^{-\sigma-1} \sim \varrho_{1}$ (respectively, $\left.\sim \varrho_{3}\right)$, if $\left|\varrho_{1}\right|<\varepsilon$ (respectively, $\left.\left|\varrho_{3}\right|<\varepsilon\right)$. Let $\left(c_{0}, c_{x}\right)=(1, c)$ with $0<|c|<R_{0}$. If $\left|\gamma_{-}^{x}\right|=\mid(\sigma+$ 
$\left.2 \theta_{x}+\theta_{\infty}\right) /(4 c) \mid<R_{0} / 4$, then $\left|\sigma+2 \theta_{x}+\theta_{\infty}\right|<R_{0}^{2}$ and $\left|\gamma_{ \pm}^{0}\right|,\left|\gamma_{+}^{x}\right|<R_{*}, R_{*}=R_{*}\left(\theta_{0}, \theta_{x}, \theta_{\infty}, R_{0}\right)$ being some number depending on $\left(\theta_{0}, \theta_{x}, \theta_{\infty}, R_{0}\right)$ only. Choose $\varepsilon=\varepsilon_{0}$ in such a way that (6.3) is valid uniformly in $\left(\gamma_{ \pm}^{0}, \gamma_{ \pm}^{x}\right)$ satisfying $\left|\gamma_{ \pm}^{0}\right|,\left|\gamma_{+}^{x}\right|<R_{*},\left|\gamma_{-}^{x}\right|<R_{0} / 4$. Then there exists a domain consisting of $(c, \sigma)$ such that $\left|\left(\sigma+2 \theta_{x}+\theta_{\infty}\right) / c\right|<R_{0}, \sigma \neq \pm 2 \theta_{0}+\theta_{\infty}, 2 \theta_{x}-\theta_{\infty}$ and $\left|\varrho_{1}\right|=\left|4 c /\left(\sigma+2 \theta_{0}-\theta_{\infty}\right)\right|<\varepsilon_{0}$, since $\theta_{0}-\theta_{x}-\theta_{\infty} \neq 0$. For such $(c, \sigma), y(c, \sigma, x)$ has a sequence of zeros $\left\{x_{m}^{(0)}\right\}$ with $\rho_{0}(\sigma)=\varrho_{1} / c=-4 /\left(\sigma+2 \theta_{0}-\theta_{\infty}\right)$. For $\left(c_{0}, c_{x}\right)=(1 / c, 1)$ with $0<|c|<R_{0}$, we obtain another sequence of zeros with $\rho_{0}(\sigma)=\varrho_{3} / c=-4 /\left(\sigma+2 \theta_{x}+\theta_{\infty}\right)$. Thus the assertion (1) of Theorem 2.26 has been verified. The second assertion is shown by an analogous argument about a pole $x^{(\infty)}$ such that $\mathrm{e}^{x^{(\infty)}}\left(x^{(\infty)}\right)^{\sigma-1} \sim \tilde{\varrho}_{1}$ or $\sim \tilde{\varrho}_{3}$ in the domain $\varepsilon^{-1}|x|^{-1}<\left|\mathrm{e}^{x} x^{\sigma-1}\right|<\varepsilon$. By putting $\sigma=\sigma_{0}=-2 \theta_{x}-\theta_{\infty}$ or $\sigma_{0}^{\prime}=2 \theta_{0}+\theta_{\infty}$, and observing $\mathrm{e}^{-x} x^{-\left(\sigma-\theta_{\infty}\right) / 2} g_{+}=\gamma_{+}^{x}-\left(\gamma_{+}^{0} / 2\right)\left(\sigma_{0}+\theta_{\infty}\right) \mathrm{e}^{-x} x^{-\sigma-1}+O\left(x^{-1}\right)$ and so on, we deduce Theorem 2.27.

\section{Proofs of the results on the monodromy data}

To show Theorem 2.10 we compute the monodromy matrices $M_{0}, M_{x}$ with respect to solution (2.1) of linear system (1.1) by matching perturbed solutions as $x \rightarrow \infty$. Note that, by Theorem 2.1,

$$
\begin{aligned}
& A_{0}(\mathbf{c}, \sigma, x) \sim \frac{1}{4}\left(\sigma-\theta_{\infty}\right) J+\gamma_{+}^{0} x^{-\left(\sigma+\theta_{\infty}\right) / 2} \Delta_{+}+\gamma_{-}^{0} x^{\left(\sigma+\theta_{\infty}\right) / 2} \Delta_{-}, \\
& A_{x}(\mathbf{c}, \sigma, x) \sim-\frac{1}{4}\left(\sigma+\theta_{\infty}\right) J+\gamma_{+}^{x} \mathrm{e}^{x} x^{\left(\sigma-\theta_{\infty}\right) / 2} \Delta_{+}+\gamma_{-}^{x} \mathrm{e}^{-x} x^{-\left(\sigma-\theta_{\infty}\right) / 2} \Delta_{-},
\end{aligned}
$$

if $\mathrm{e}^{x} x^{\sigma-1}, \mathrm{e}^{-x} x^{-\sigma-1}=o(1)$. In what follows we suppose that $\arg x \sim \pi / 2$ and that

$$
\left|\mathrm{e}^{x} x^{\sigma}\right|,\left|\mathrm{e}^{-x} x^{-\sigma}\right| \ll 1
$$

as $x \rightarrow \infty$. By $Y=\mathrm{e}^{(x / 4) J} x^{-\left(\theta_{\infty} / 4\right) J} \hat{Y}$, system (1.1) is changed into

$$
\frac{\mathrm{d} \hat{Y}}{\mathrm{~d} \lambda}=\left(\frac{\hat{A}_{0}}{\lambda}+\frac{\hat{A}_{x}}{\lambda-x}+\frac{J}{2}\right) \hat{Y}
$$

with

$$
\begin{aligned}
\hat{A}_{0}= & \left(\left(\sigma-\theta_{\infty}\right) / 4+O\left(x^{-1}\right)\right) J \\
& +\left(\gamma_{+}^{0}+O\left(x^{-1}\right)\right) \mathrm{e}^{-x / 2} x^{-\sigma / 2} \Delta_{+}+\left(\gamma_{-}^{0}+O\left(x^{-1}\right)\right) \mathrm{e}^{x / 2} x^{\sigma / 2} \Delta_{-}, \\
\hat{A}_{x}= & \left(-\left(\sigma+\theta_{\infty}\right) / 4+O\left(x^{-1}\right)\right) J \\
& +\left(\gamma_{+}^{x}+O\left(x^{-1}\right)\right) \mathrm{e}^{x / 2} x^{\sigma / 2} \Delta_{+}+\left(\gamma_{-}^{x}+O\left(x^{-1}\right)\right) \mathrm{e}^{-x / 2} x^{-\sigma / 2} \Delta_{-} .
\end{aligned}
$$

\subsection{Approximate equation}

As long as $|\lambda|>|x|^{1 / 2},|\lambda-x|>|x|^{1 / 2}$, the eigenvalues of $A_{*}=\hat{A}_{0} / \lambda+\hat{A}_{x} /(\lambda-x)+J / 2$ are $\pm \mu(x, \lambda)$ with

$$
\mu(x, \lambda)=\frac{1}{2}+\frac{\left(\sigma-\theta_{\infty}\right) / 4+O\left(x^{-1}\right)}{\lambda}-\frac{\left(\sigma+\theta_{\infty}\right) / 4+O\left(x^{-1}\right)}{\lambda-x}+O\left(|\lambda|^{-2}+|\lambda-x|^{-2}\right),
$$

and by $\hat{Y}=\left(\mu(x, \lambda) J+A_{*}\right) Z=\left(J+O\left(|\lambda|^{-1}+|\lambda-x|^{-1}\right)\right) Z$ system (7.2) is reduced to

$$
\frac{\mathrm{d} Z}{\mathrm{~d} \lambda}=(\mu(x, \lambda) J+H(x, \lambda)) Z, \quad H(x, \lambda)=\left(h_{i j}(x, \lambda)\right) \ll|\lambda|^{-2}+|\lambda-x|^{-2} .
$$


Lemma 7.1. Let $\Sigma_{\pi / 2}(x)$ be a sector given by

$$
|\arg \lambda-\pi / 2|<\pi, \quad|\arg (\lambda-x)-\pi / 2|<\pi / 4, \quad|\lambda-x|>|x|^{1 / 2},
$$

and $\Sigma_{3 \pi / 2}(0)$ one given by

$$
|\arg \lambda-3 \pi / 2|<\pi / 4, \quad|\lambda|>|x|^{1 / 2} .
$$

Then (7.2) admits the matrix solution

$$
Z_{\mathrm{WKB}}^{x}(x, \lambda)=\left(J+O\left(|\lambda|^{-1}+|\lambda-x|^{-1}\right)\right) \mathrm{e}^{(\lambda / 2) J} \lambda^{\alpha(x) J}(\lambda-x)^{\beta(x) J}
$$

with

$$
\alpha(x)=\left(\sigma-\theta_{\infty}\right) / 4+O\left(x^{-1}\right), \quad \beta(x)=-\left(\sigma+\theta_{\infty}\right) / 4+O\left(x^{-1}\right)
$$

uniformly in sufficiently large $x$ as $\lambda \rightarrow \infty$ through $\Sigma_{\pi / 2}(x)$, and the solution $Z_{\mathrm{WKB}}^{0}(x, \lambda)$ having an asymptotic representation of the same form as $\lambda \rightarrow \infty$ through $\Sigma_{3 \pi / 2}(0)$.

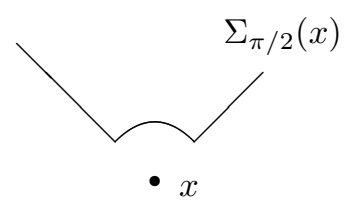

- 0

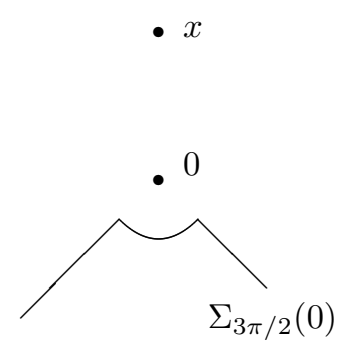

Figure 7.1. Sectors $\Sigma_{\pi / 2}(x)$ and $\Sigma_{3 \pi / 2}(0)$.

Proof. By $Z=\left(I+p \Delta_{+}\right) Z_{*}$ system (7.2) is taken to

$$
\frac{\mathrm{d} Z_{*}}{\mathrm{~d} \lambda}=\left(\mu(x, \lambda) J+H(x, \lambda)-h_{21} p J+h_{*} \Delta_{+}\right) Z_{*}
$$

with

$$
h_{*}=2 \mu(x, \lambda) p-\mathrm{d} p / \mathrm{d} \lambda+\left(h_{11}-h_{22}\right) p-h_{21} p^{2} .
$$

From every point in $\Sigma_{\pi / 2}(x)$ one may draw a line in $\Sigma_{\pi / 2}(x)$ in such a way that $\operatorname{Re} \lambda \rightarrow \infty$, and hence there exists $p=p(x, \lambda)$ such that $h_{12}+h_{*}=0$ and that $p(x, \lambda) \ll|\lambda|^{-2}+|\lambda-x|^{-2}$ (cf. Lemma 4.1 and Remark 4.3). As a result the coefficient matrix becomes of lower-triangular form. We apply a suitable further transformation of the form $Z_{*}=\left(I+q \Delta_{-}\right) Z_{* *}$ with $q=$ $q(x, \lambda) \ll|\lambda|^{-2}+|\lambda-x|^{-2}$ to get the diagonal system

$$
\frac{\mathrm{d} Z_{* *}}{\mathrm{~d} \lambda}=\left(\mu(x, \lambda) J+\operatorname{diag}\left[\tilde{h}_{1}(x, \lambda), \tilde{h}_{2}(x, \lambda)\right]\right) Z_{* *}
$$

with $\tilde{h}_{1}(x, \lambda), \tilde{h}_{2}(x, \lambda) \ll|\lambda|^{-2}+|\lambda-x|^{-2}$, from which the desired solution immediately follows.

Remark 7.2. In the sector $\Sigma_{-\pi / 2}(0):|\arg \lambda+\pi / 2|<\pi / 4,|\lambda|>|x|^{1 / 2}$ as well, (7.2) admits the solution $\hat{Z}_{\mathrm{WKB}}^{0}(x, \lambda)$ with an asymptotic representation of the same form. 
Remark 7.3. The matrix function $Z_{\mathrm{WKB}}^{x}(x, \lambda)$ or $W_{\mathrm{WKB}}^{0}(x, \lambda)$, which corresponds to $\Psi_{q}(\lambda)$ given by [2, equation (7.10)], is essentially a WKB solution. The representation for it remains valid also in a suitably extended sector with opening angle $\pi-\delta$. Furthermore, e.g., the first column of $W_{\mathrm{WKB}}^{x}(x, \lambda)$ is a vector solution in a domain with the properties:

(i) $|\arg (\lambda-x)|,|\arg \lambda|<3 \pi / 2-\delta,|\lambda|,|\lambda-x|>|x|^{1 / 2}$;

(ii) from every point in the domain one may draw a line contained in it and satisfying $\operatorname{Re} \lambda \rightarrow \infty$.

\subsection{Local equation}

If $|\lambda|<2|x|^{1 / 2}$, system $(7.2)$ is written as

$$
\frac{\mathrm{d} \hat{Y}}{\mathrm{~d} \lambda}=\left(\frac{J}{2}+\frac{\hat{A}_{0}}{\lambda}+O\left(x^{-1}\right)\right) \hat{Y}
$$

under (7.1). This is equivalent to

$$
\frac{\mathrm{d} U}{\mathrm{~d} \lambda}=\left(\frac{J}{2}+\frac{\Lambda}{\lambda}+E(x, \lambda)\right) U, \quad \Lambda:=\frac{1}{4}\left(\sigma-\theta_{\infty}\right) J+\gamma_{+}^{0} c_{0}^{-1} \Delta_{+}+\gamma_{-}^{0} c_{0} \Delta_{-}
$$

with $E(x, \lambda) \ll x^{-1}$ for $|\lambda|<2|x|^{1 / 2}$, in which

$$
U=\mathrm{e}^{(x / 4) J} x^{(\sigma / 4) J} c_{0}^{-J / 2} \hat{Y} \text {. }
$$

System (7.4) is a perturbation of the Whittaker system

$$
\frac{\mathrm{d} W}{\mathrm{~d} \lambda}=\left(\frac{J}{2}+\frac{\Lambda}{\lambda}\right) W
$$

which admits the matrix solution

$$
W_{\infty}(\lambda)=\left(I+O\left(\lambda^{-1}\right)\right) \mathrm{e}^{(\lambda / 2) J} \lambda^{\left(\left(\sigma-\theta_{\infty}\right) / 4\right) J}
$$

as $\lambda \rightarrow \infty$ through the sector $|\arg \lambda-\pi / 2|<\pi-\delta$. By $W_{\infty}^{*}(\lambda)$ and $W_{\infty}^{* *}(\lambda)$ we denote the solutions of (7.5) having an asymptotic representation of the same form in the neighbouring sectors $|\arg \lambda+\pi / 2|<\pi-\delta$ and $|\arg \lambda-3 \pi / 2|<\pi-\delta$, respectively.

Lemma 7.4. In the domain $|\arg \lambda-3 \pi / 2|<\pi / 4, \log |x|^{1 / 4}<|\lambda|<2|x|^{1 / 2}$, system (7.4) admits the matrix solution

$$
U_{\text {out }}(x, \lambda)=\left(I+U_{\text {out }}^{*}(x, \lambda)\right) \mathrm{e}^{(\lambda / 2) J} \lambda^{\left(\left(\sigma-\theta_{\infty}\right) / 4\right) J}
$$

with $U_{\text {out }}^{*}(x, \lambda) \ll(\log |x|)^{-1}$.

Proof. Write $W_{\infty}^{* *}(\lambda)=\bar{W}(\lambda) \mathrm{e}^{(\lambda / 2) J} \lambda^{\left(\left(\sigma-\theta_{\infty}\right) / 4\right) J}$ with $\bar{W}(\lambda)=I+O\left(\lambda^{-1}\right)$ as $\lambda \rightarrow \infty$. Since $W=\bar{W}(\lambda) \hat{W}$ reduces $(7.5)$ to $\mathrm{d} \hat{W} / \mathrm{d} \lambda=\left(1 / 2+\left(\sigma-\theta_{\infty}\right) /(4 \lambda)\right) J \hat{W}$, it is easy to see that, by $U=\bar{W}(\lambda) \hat{U},(7.4)$ becomes

$$
\frac{\mathrm{d} \hat{U}}{\mathrm{~d} \lambda}=\left(\left(\frac{1}{2}+\frac{\sigma-\theta_{\infty}}{4 \lambda}\right) J+O\left(x^{-1}\right)\right) \hat{U}
$$


and $\bar{W}(\lambda)=I+O\left((\log |x|)^{-1}\right)$, if $|\arg \lambda-3 \pi / 2|<\pi / 4, \log |x|^{1 / 4}<|\lambda|<2|x|^{1 / 2}$. By the same argument as in the proof of Lemma 7.1, we may find a transformation of the form $\hat{U}=$ $\left(I+O\left(x^{-1}\right)\right) \tilde{U}$ such that this system is changed into

$$
\frac{\mathrm{d} \tilde{U}}{\mathrm{~d} \lambda}=\left(\left(\frac{1}{2}+\frac{\sigma-\theta_{\infty}}{4 \lambda}\right) J+\operatorname{diag}\left[e_{1}(x, \lambda), e_{2}(x, \lambda)\right]\right) \tilde{U}
$$

with $e_{1}(x, \lambda), e_{2}(x, \lambda) \ll x^{-1}$, which has a matrix solution given by

$$
\tilde{U}=\operatorname{diag}\left[\hat{e}_{1}(x, \lambda), \hat{e}_{2}(x, \lambda)\right] \mathrm{e}^{(\lambda / 2) J} \lambda^{\left(\left(\sigma-\theta_{\infty}\right) / 4\right) J}
$$

with $\hat{e}_{1}(x, \lambda)-1, \hat{e}_{2}(x, \lambda)-1 \ll \lambda x^{-1} \ll x^{-1 / 2}$. Thus we obtain the lemma.

Lemma 7.5. In the domain $|\arg \lambda-3 \pi / 2|<\pi / 4,1<|\lambda|<\log |x|^{1 / 3}$, system (7.4) admits the matrix solution

$$
U_{\text {in }}(x, \lambda)=\left(I+U_{\text {in }}^{*}(x, \lambda)\right) W_{\infty}^{* *}(\lambda)
$$

with $U_{\text {in }}^{*}(x, \lambda) \ll x^{-1 / 4}$.

Proof. By $U=W_{\infty}^{* *}(\lambda) \bar{U}$ system (7.4) is reduced to

$$
\frac{\mathrm{d} \bar{U}}{\mathrm{~d} \lambda}=W_{\infty}^{* *}(\lambda)^{-1} E(x, \lambda) W_{\infty}^{* *}(\lambda) \bar{U} .
$$

Here $W_{\infty}^{* *}(\lambda)^{-1} E(x, \lambda) W_{\infty}^{* *}(\lambda) \ll x^{-2 / 3+\epsilon}$ for $1<|\lambda|<\log |x|^{1 / 3}, \epsilon$ being any positive number, since $W_{\infty}^{* *}(\lambda)^{ \pm} \ll x^{1 / 6+\epsilon}$. Using Gronwall's inequality, we may show that there exists a matrix solution such that $\bar{U}=I+\bar{U}^{*}(x, \lambda)$ with $\bar{U}^{*}(x, \lambda) \ll x^{-2 / 3+\epsilon}$. Then

$$
U=W_{\infty}^{* *}(\lambda)\left(I+\bar{U}^{*}(x, \lambda)\right)=\left(I+W_{\infty}^{* *}(\lambda) \bar{U}^{*}(x, \lambda) W_{\infty}^{* *}(\lambda)^{-1}\right) W_{\infty}^{* *}(\lambda)
$$

solves (7.4). Since $W_{\infty}^{* *}(\lambda) \bar{U}^{*}(x, \lambda) W_{\infty}^{* *}(\lambda)^{-1} \ll x^{-1 / 3+\epsilon}$, this is a desired solution as in the lemma.

Remark 7.6. In the domain $|\arg \lambda+\pi / 2|<\pi / 4, \log |x|^{1 / 4}<|\lambda|<2|x|^{1 / 2}$ (respectively, $1<|\lambda|<\log |x|^{1 / 3}$ ) as well, we have the solution $\hat{U}_{\text {out }}(x, \lambda)$ (respectively, $\hat{U}_{\text {in }}(x, \lambda)$ ) with an analogous property, which is obtained by using $W_{\infty}^{*}(\lambda)$.

\subsection{Whittaker system}

The right-hand side of $W_{\infty}(\lambda)$ (cf. (7.6)) is given by

$$
\left(\begin{array}{cc}
\mathrm{e}^{\pi \mathrm{i}\left(\sigma-\theta_{\infty}+2\right) / 4} W_{\left(\sigma-\theta_{\infty}+2\right) / 4, \theta_{0} / 2}\left(\mathrm{e}^{-\pi \mathrm{i}} \lambda\right) & -\vartheta_{+} W_{-\left(\sigma-\theta_{\infty}+2\right) / 4, \theta_{0} / 2}(\lambda) \\
\vartheta_{-} \mathrm{e}^{\pi \mathrm{i}\left(\sigma-\theta_{\infty}+2\right) / 4} W_{\left(\sigma-\theta_{\infty}-2\right) / 4, \theta_{0} / 2}\left(\mathrm{e}^{-\pi \mathrm{i}} \lambda\right) & W_{-\left(\sigma-\theta_{\infty}-2\right) / 4, \theta_{0} / 2}(\lambda)
\end{array}\right) \lambda^{-1 / 2},
$$

where $\vartheta_{+}=\left(\sigma-\theta_{\infty}+2 \theta_{0}\right) / 4, \vartheta_{-}=\left(\sigma-\theta_{\infty}-2 \theta_{0}\right) / 4$, and $W_{\kappa, \nu}(z)$ is the Whittaker function such that $W_{\kappa, \nu}(z) \sim \mathrm{e}^{-z / 2} z^{\kappa}$ as $z \rightarrow \infty$ through the sector $|\arg z|<3 \pi / 2$ (cf. [1, formula (13.1.33)], [5, Section 6.9], [14, equation (3.10)]). Around $\lambda=0,(7.5)$ admits the matrix solution

$$
W_{0}(\lambda)=G_{0}(I+O(\lambda)) \lambda^{\left(\theta_{0} / 2\right) J} \lambda^{\Delta_{*}},
$$

where $G_{0} \in \mathrm{GL}_{2}(\mathbb{C})$, and $\Delta_{*}$ denotes 0 if $\theta_{0} \notin \mathbb{Z}, \Delta_{+}$if $\theta_{0} \in \mathbb{N} \cup\{0\}$, and $\Delta_{-}$if $-\theta_{0} \in \mathbb{N}$.

Let us compute connection formulas and Stokes multipliers. Using the formula

$$
z^{-1 / 2} W_{\kappa, \nu}(z)=\frac{\Gamma(-2 \nu) z^{\nu}}{\Gamma(1 / 2-\nu-\kappa)}(1+O(z))+\frac{\Gamma(2 \nu) z^{-\nu}}{\Gamma(1 / 2+\nu-\kappa)}(1+O(z))
$$

near $z=0$ (cf. [1, formulas (13.1.2), (13.1.32), (13.1.34)]), we have 
Proposition 7.7. If $\theta_{0} \notin \mathbb{Z}$, then $W_{\infty}(\lambda)=W_{0}(\lambda) V_{0}$, where $V_{0}$ is the matrix as in Theorem 2.10.

Furthermore, if $2 \nu \in \mathbb{Z} \backslash\{0\}$,

$$
\begin{aligned}
W_{\kappa, \nu}(z)= & \frac{(-1)^{1+|2 \nu|} z^{1 / 2+|\nu|}}{|2 \nu| ! \Gamma(1 / 2-|\nu|-\kappa)}((1+O(z)) \log z+\psi(1 / 2+|\nu|-\kappa) \\
& -\psi(1)-\psi(1+|2 \nu|)+O(z))+\frac{(|2 \nu|-1) ! z^{1 / 2-|\nu|}(1+O(z))}{\Gamma(1 / 2+|\nu|-\kappa)}
\end{aligned}
$$

and

$$
W_{\kappa, 0}(z)=-\frac{z^{1 / 2}}{\Gamma(1 / 2-\kappa)}((1+O(z)) \log z+\psi(1 / 2-\kappa)-2 \psi(1)+O(z))
$$

near $z=0$ (cf. [1, formulas (13.1.6), (13.1.7), (13.1.33)]). From these formulas we have

Proposition 7.8. If $\theta_{0} \in \mathbb{Z}$, then $W_{\infty}(\lambda)=W_{0}(\lambda) \hat{V}_{0}$, where $\hat{V}_{0}$ is the matrix as in Theorem 2.11.

To calculate the relation between $W_{\infty}(\lambda)$ and $W_{\infty}^{*}(\lambda)$, we use

$$
\begin{aligned}
W_{\kappa, \nu}\left(\mathrm{e}^{-\pi \mathrm{i}} \lambda\right)= & \mathrm{e}^{-\pi \mathrm{i}(\nu+1 / 2)} \mathrm{e}^{\lambda / 2} \lambda^{\nu+1 / 2}\left(\frac{\left(1-\mathrm{e}^{4 \pi \mathrm{i} \nu}\right) \Gamma(-2 \nu)}{\Gamma(1 / 2-\nu-\kappa)} M\left(\nu-\kappa+1 / 2,2 \nu+1, \mathrm{e}^{\pi \mathrm{i}} \lambda\right)\right. \\
& \left.+\mathrm{e}^{4 \pi \mathrm{i} \nu} U\left(\nu-\kappa+1 / 2,2 \nu+1, \mathrm{e}^{\pi \mathrm{i}} \lambda\right)\right),
\end{aligned}
$$

which is obtained from $[1$, formulas $(13.1 .10),(13.1 .33)]$ with $n=-1$. Here, by [1, formulas (13.5.1), (13.5.2)]

$$
\begin{aligned}
M\left(\nu-\kappa+1 / 2,2 \nu+1, \mathrm{e}^{\pi \mathrm{i}} \lambda\right)= & \frac{\Gamma(2 \nu+1) \lambda^{-(\nu-\kappa+1 / 2)}}{\Gamma(\nu+\kappa+1 / 2)}\left(1+O\left(\lambda^{-1}\right)\right) \\
& +\frac{\Gamma(2 \nu+1)}{\Gamma(\nu-\kappa+1 / 2)} \mathrm{e}^{-\lambda}\left(\mathrm{e}^{\pi \mathrm{i}} \lambda\right)^{-(\nu+\kappa+1 / 2)}\left(1+O\left(\lambda^{-1}\right)\right), \\
U\left(\nu-\kappa+1 / 2,2 \nu+1, \mathrm{e}^{\pi \mathrm{i}} \lambda\right)= & \left(\mathrm{e}^{\pi \mathrm{i}} \lambda\right)^{-(\nu-\kappa+1 / 2)}\left(1+O\left(\lambda^{-1}\right)\right),
\end{aligned}
$$

in the sector $-3 \pi / 2<\arg \lambda<\pi / 2$. From [1, formulas (13.1.10), (13.1.33)] with $n=1$, it follows that

$$
\begin{aligned}
W_{\kappa, \nu}(\lambda)= & \mathrm{e}^{-\lambda / 2} \lambda^{\nu+1 / 2}\left(\frac{\left(1-\mathrm{e}^{-4 \pi \mathrm{i} \nu}\right) \Gamma(-2 \nu)}{\Gamma(1 / 2-\nu-\kappa)} M\left(\nu-\kappa+1 / 2,2 \nu+1, \mathrm{e}^{-2 \pi \mathrm{i}} \lambda\right)\right. \\
& \left.+\mathrm{e}^{-4 \pi \mathrm{i} \nu} U\left(\nu-\kappa+1 / 2,2 \nu+1, \mathrm{e}^{-2 \pi \mathrm{i}} \lambda\right)\right)
\end{aligned}
$$

which yields the relation between $W_{\infty}(\lambda)$ and $W_{\infty}^{* *}(\lambda)$.

Proposition 7.9. We have $W_{\infty}(\lambda)=W_{\infty}^{*}(\lambda) S_{*}$ and $W_{\infty}(\lambda)=W_{\infty}^{* *}(\lambda) S_{* *}$, where $S_{*}$ and $S_{* *}$ are the matrices as in Theorem 2.10 .

\subsection{Completion of the proofs of Theorems 2.10 and 2.11}

Recall the solution $Y(x, \lambda)=\left(I+O\left(\lambda^{-1}\right)\right) \mathrm{e}^{(\lambda / 2) J} \lambda^{-\left(\theta_{\infty} / 2\right) J}$ of $(1.1)$ as $\lambda \rightarrow \infty$ through the sector $|\arg \lambda-\pi / 2|<\pi$ and the monodromy matrices $M_{0}, M_{x}$ defined by the analytic continuation of $Y(x, \lambda)$ along the loops $l_{0}, l_{x}$ as described in Section 2.2. Furthermore, for the solutions $Y_{1}(x, \lambda)$ and $Y_{2}(x, \lambda)$, respectively, in $|\arg \lambda+\pi / 2|<\pi$ and $|\arg \lambda-3 \pi / 2|<\pi$, the Stokes multipliers $S_{1}$ and $S_{2}$ are given by $Y(x, \lambda)=Y_{1}(x, \lambda) S_{1}, Y_{2}(x, \lambda)=Y(x, \lambda) S_{2}$. 


\subsubsection{Derivation of $M_{0}$}

To compute $M_{0}$ let us examine the analytic continuation for $Y(x, \lambda)$ along $l_{0}$ by the matching procedure carried out according to the following scheme:

$$
Y(x, \lambda)=Y_{2}(x, \lambda) S_{2}^{-1} \longleftrightarrow Z_{\mathrm{WKB}}^{0}(x, \lambda) \longleftrightarrow U_{\text {out }}(x, \lambda) \longleftrightarrow U_{\mathrm{in}}(x, \lambda)
$$

(cf. Lemmas 7.1, 7.4 and 7.5).

Suppose that $x$ satisfies $\arg x \sim \pi / 2$ and (7.1), and that the starting point $\lambda_{\text {st }}$ of $l_{0}$ has the properties $\arg \lambda_{\text {st }} \sim \pi / 2, \arg \left(\lambda_{\text {st }}-x\right) \sim \pi / 2$ and $\left|\lambda_{\text {st }}\right|>2|x|$. Then the part of $l_{0}$ from $\lambda_{\text {st }}$ up to a point near $\lambda=0$ may be regarded as $\Gamma_{\text {left }} \cup L_{-}$with $\Gamma_{\text {left }}: \lambda=\left|\lambda_{\text {st }}\right| \mathrm{e}^{\mathrm{i} t}(\pi / 2 \leq t \leq 3 \pi / 2)$ and $L_{-}: \lambda=\mathrm{it}\left(-\left|\lambda_{\mathrm{st}}\right| \leq t \leq-1\right)$.

1-st step: Continue $Y(x, \lambda)$ along the arc $\Gamma_{\text {left }}$ entering into $\Sigma_{3 \pi / 2}(0) \cap\{|\lambda|>2|x|\}$, in which $|\arg (\lambda-x)-3 \pi / 2|<\pi / 4$ (cf. Lemma 7.1 and Fig. 7.2(a)).

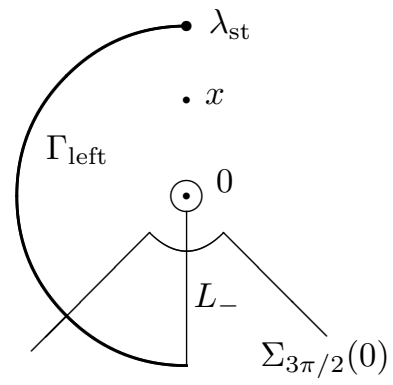

(a) $l_{0}$

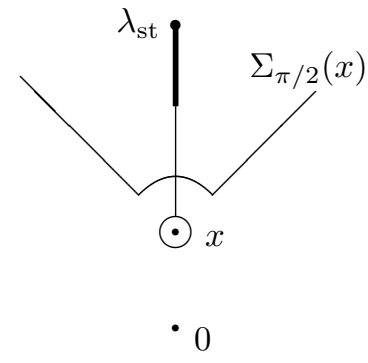

(b) $l_{x}$

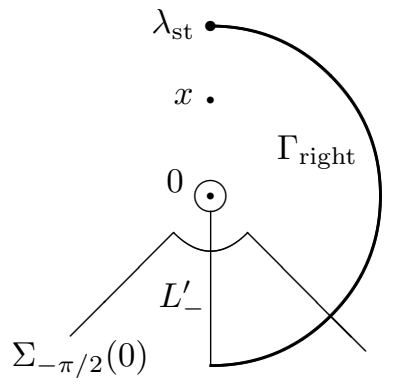

(c) $l_{x}^{-1} l_{0} l_{x}$

Figure 7.2. $l_{0}, l_{x}$ and $l_{x}^{-1} l_{0} l_{x}$.

Let us match $Y_{2}(x, \lambda)$ with $Z_{\mathrm{WKB}}^{0}(x, \lambda)$ in this domain. Since $Z_{\mathrm{WKB}}^{0}(x, \lambda)$ solves $(7.2)$ that follows from (1.1) by $Y=\mathrm{e}^{(x / 4) J} x^{-\left(\theta_{\infty} / 4\right) J} \hat{Y}$, we have

$$
Y_{2}(x, \lambda)=\mathrm{e}^{(x / 4) J} x^{-\left(\theta_{\infty} / 4\right) J} Z_{\mathrm{WKB}}^{0}(x, \lambda) \Upsilon_{1}(x)
$$

with $\Upsilon_{1}(x) \in \mathrm{SL}_{2}(\mathbb{C})$. By Lemma 7.1, the right-hand side is

$$
\begin{aligned}
& \mathrm{e}^{(x / 4) J} x^{-\left(\theta_{\infty} / 4\right) J}\left(J+O\left(\lambda^{-1}\right)\right) \mathrm{e}^{(\lambda / 2) J} \lambda^{\alpha(x) J}(\lambda-x)^{\beta(x) J} \Upsilon_{1}(x) \\
& \quad=\left(I+O\left(\lambda^{-1 / 2}\right)\right) \mathrm{e}^{(\lambda / 2) J} \lambda^{-\left(\theta_{\infty} / 2+O\left(x^{-1}\right)\right) J} \mathrm{e}^{(x / 4) J} x^{-\left(\theta_{\infty} / 4\right) J} J \Upsilon_{1}(x),
\end{aligned}
$$

provided that, e.g., $|\lambda|^{1 / 2} \gg|x|^{\left(|\sigma|+\left|\theta_{\infty}\right|\right) / 2} \gg\left|\mathrm{e}^{x / 2} x^{-\theta_{\infty} / 2}\right|^{ \pm 1}$ (cf. (7.1)). Under $|\lambda| \ll \exp \left(|x|^{1 / 2}\right)$, which implies $\lambda^{O\left(x^{-1}\right)}=1+o(1)$, from (7.8) we conclude

$$
\Upsilon_{1}(x)=\mathrm{e}^{-(x / 4) J} x^{\left(\theta_{\infty} / 4\right) J}(J+o(1)) .
$$

2-nd step: The line $L_{-}$is contained in the sector $|\lambda-3 \pi / 2|<\pi / 4$. Recall that $U=$ $\mathrm{e}^{(x / 4) J} x^{(\sigma / 4) J} c_{0}^{-J / 2} \hat{Y}$ takes (7.2) to (7.4). Suppose that

$$
\mathrm{e}^{(x / 4) J} x^{(\sigma / 4) J} c_{0}^{-J / 2} Z_{\mathrm{WKB}}^{0}(x, \lambda)=U_{\text {out }}(x, \lambda) \Upsilon_{2}(x)
$$

in the domain $|\arg \lambda-3 \pi / 2|<\pi / 4,|x|^{1 / 2}<|\lambda|<2|x|^{1 / 2}$. By (7.1) the left-hand side is

$$
\begin{aligned}
\mathrm{e}^{(x / 4) J} & x^{(\sigma / 4) J} c_{0}^{-J / 2}\left(J+O\left(\lambda^{-1}\right)\right) \mathrm{e}^{(\lambda / 2) J} \lambda^{\alpha(x) J}\left(\mathrm{e}^{\pi \mathrm{i}} x(1-\lambda / x)\right)^{\beta(x) J} \\
= & \left(I+O\left(\lambda^{-1}\right)\right) \mathrm{e}^{(\lambda / 2) J} \lambda^{\left(\left(\sigma-\theta_{\infty}\right) / 4+O\left(x^{-1}\right)\right) J}(1-\lambda / x)^{\beta(x) J} \mathrm{e}^{\beta(x) \pi \mathrm{i} J} \\
& \times \mathrm{e}^{(x / 4) J} x^{\left(-\theta_{\infty} / 4+O\left(x^{-1}\right)\right) J} c_{0}^{-J / 2} J,
\end{aligned}
$$


since $|\arg (\lambda-x)-3 \pi / 2|<\pi / 4, \arg x \sim \pi / 2$. Using Lemma 7.4, we derive

$$
\Upsilon_{2}(x)=\mathrm{e}^{-\left(\left(\sigma+\theta_{\infty}\right) \pi \mathrm{i} / 4\right) J} \mathrm{e}^{(x / 4) J} x^{-\left(\theta_{\infty} / 4\right) J} c_{0}^{-J / 2}(J+o(1)) .
$$

3-rd step: In the domain $|\arg \lambda-3 \pi / 2|<\pi / 4, \log |x|^{1 / 4}<|\lambda|<\log |x|^{1 / 3}$, by Lemmas 7.4 and 7.5 , we have

$$
U_{\text {out }}(x, \lambda)=U_{\text {in }}(x, \lambda) \Upsilon_{3}(x)
$$

with $\Upsilon_{3}(x)=I+o(1)$. By Lemma 7.4 and Propositions 7.7 through 7.9,

$$
U_{\text {in }}(x, \lambda)=\left(I+O\left(x^{-1}\right)\right) W_{\infty}(\lambda) S_{* *}^{-1}=\left(I+O\left(x^{-1}\right)\right) W_{0}(\lambda) V_{*} S_{* *}^{-1}
$$

in the domain $|\arg \lambda-3 \pi / 2|<\pi / 4,1<|\lambda|<2$, where $V_{*}=V_{0}$ if $\theta_{0} \notin \mathbb{Z}$ (respectively, $V_{*}=\hat{V}_{0}$ if $\theta_{0} \in \mathbb{Z}$ ). From (7.8), (7.9), (7.10) and (7.11), as a result of the matching procedure we obtain the following connection formula:

$$
Y(x, \lambda)=x^{-\left(\left(\sigma+\theta_{\infty}\right) / 4\right) J} c_{0}^{J / 2}\left(I+O\left(x^{-1}\right)\right) G_{0}(I+O(\lambda)) \lambda^{\left(\theta_{\infty} / 2\right) J} \lambda^{\Delta_{*}} \Upsilon_{0}(x)
$$

with

$$
\Upsilon_{0}(x)=V_{*} S_{* *}^{-1} \Upsilon_{3}(x) \Upsilon_{2}(x) \Upsilon_{1}(x) S_{2}^{-1}=V_{*} S_{* *}^{-1} \mathrm{e}^{-\pi \mathrm{i}\left(\left(\sigma+\theta_{\infty}\right) / 4\right) J} c_{0}^{-J / 2}(I+o(1)) S_{2}^{-1}
$$

around $\lambda=0$ as $|x| \rightarrow \infty, \arg x \sim \pi / 2$. Since $M_{0}$ does not depend on $x$, we derive

$$
M_{0}=S_{2}\left(C_{0}^{2}\right)^{-1} \mathrm{e}^{\pi \mathrm{i} \theta_{0} J} C_{0}^{2} S_{2}^{-1}, \quad C_{0}^{2}=V_{0} S_{* *}^{-1} \mathrm{e}^{-\pi \mathrm{i}\left(\left(\sigma+\theta_{\infty}\right) / 4\right) J} c_{0}^{-J / 2}
$$

if $\theta_{0} \notin \mathbb{Z}$, which is the second relation in (2.3). The case $\theta_{0} \in \mathbb{Z}$ is treated similarly, and that of (2.5) follows.

\subsubsection{Derivation of $M_{x} M_{0} M_{x}^{-1}$}

The curve $\Gamma_{\text {right }} \cup L_{-}^{\prime}$ issuing from $\lambda_{\text {st }}$, where $\Gamma_{\text {right }}: \lambda=\left|\lambda_{\text {st }}\right| \mathrm{e}^{\mathrm{i}(\pi / 2-t)}(0 \leq t \leq \pi)$ and $L_{-}^{\prime}: \lambda=\mathrm{i} t$ $\left(-\left|\lambda_{\text {st }}\right| \leq t \leq-1\right)$, corresponds to the part of $l_{x}^{-1} l_{0} l_{x}$ from $\lambda_{\text {st }}$ up to $\lambda=-1$ (cf. Fig. 7.2(c)). In this case the matching scheme

$$
Y(x, \lambda)=Y_{1}(x, \lambda) S_{1} \longleftrightarrow \hat{Z}_{\mathrm{WKB}}^{0}(x, \lambda) \longleftrightarrow \hat{U}_{\mathrm{out}}(x, \lambda) \longleftrightarrow \hat{U}_{\mathrm{in}}(x, \lambda)
$$

(cf. Remarks 7.2 and 7.6) yields the monodromy matrix $M_{x} M_{0} M_{x}^{-1}$. Note that $\hat{U}_{\text {out }}(x, \lambda)=(I+$ $o(1)) W_{\infty}^{*}(\lambda)(I+o(1))$, and in matching $\hat{Z}_{\mathrm{WKB}}^{0}(x, \lambda)$ with $\hat{U}_{\text {out }}(x, \lambda)$, that $\lambda-x=\mathrm{e}^{-\pi \mathrm{i}} x(1-\lambda / x)$, since $|\arg (\lambda-x)+\pi / 2|<\pi / 4, \arg x \sim \pi / 2$. Then we obtain

$$
M_{x} M_{0} M_{x}^{-1}=S_{1}^{-1}\left(C_{0}^{1}\right)^{-1} \mathrm{e}^{\pi \mathrm{i} \theta_{0} J} C_{0}^{1} S_{1}, \quad C_{0}^{1}=V_{0} S_{*}^{-1} \mathrm{e}^{\pi \mathrm{i}\left(\left(\sigma+\theta_{\infty}\right) / 4\right) J} c_{0}^{-J / 2}
$$

if $\theta_{0} \notin \mathbb{Z}$. In this way the first relations in (2.3) and (2.5) are verified.

\subsubsection{Derivation of $M_{x}$}

In the domain $|\lambda-x|<2|x|^{1 / 2}$, we write (7.2) in the form

$$
\frac{\mathrm{d} \hat{Y}}{\mathrm{~d} \lambda}=\left(\frac{J}{2}+\frac{\hat{A}_{x}}{\lambda-x}+O\left(x^{-1}\right)\right) \hat{Y}
$$


which is changed into

$$
\frac{\mathrm{d} U}{\mathrm{~d} \lambda}=\left(\frac{J}{2}+\frac{\tilde{\Lambda}}{\lambda-x}+O\left(x^{-1}\right)\right) U, \quad \tilde{\Lambda}=-\frac{1}{4}\left(\sigma+\theta_{\infty}\right) J+\gamma_{+}^{x} c_{x}^{-1} \Delta_{+}+\gamma_{-}^{x} c_{x} \Delta_{-}
$$

by $U=\mathrm{e}^{-(x / 4) J} x^{-(\sigma / 4) J} c_{x}^{-J / 2} \hat{Y}$. Then instead of (7.5) we treat

$$
\frac{\mathrm{d} W}{\mathrm{~d} \lambda}=\left(\frac{J}{2}+\frac{\tilde{\Lambda}}{\lambda-x}\right) W
$$

which has the matrix solution

$$
W_{\infty}^{x}(\lambda)=\left(I+O\left((\lambda-x)^{-1}\right)\right) \mathrm{e}^{(\lambda / 2) J}(\lambda-x)^{-\left(\left(\sigma+\theta_{\infty}\right) / 4\right) J}
$$

as $\lambda \rightarrow \infty$ through the sector $|\arg (\lambda-x)-\pi / 2|<\pi-\delta$. Around $\lambda=x$ there exists the matrix solution

$$
W_{0}^{x}(\lambda)=G_{x}(I+O(\lambda-x))(\lambda-x)^{\left(\theta_{x} / 2\right) J}(\lambda-x)^{\Delta_{*}}
$$

with $G_{x} \in \mathrm{GL}_{2}(\mathbb{C})$ and $\Delta_{*}$ as of (7.7). Then the connection formula is given by $W_{\infty}^{x}(\lambda)=$ $W_{0}^{x}(\lambda) V_{x}$ (respectively, $\left.=W_{0}^{x}(\lambda) \hat{V}_{x}\right)$ if $\theta_{x} \notin \mathbb{Z}$ (respectively, $\theta_{x} \in \mathbb{Z}$ ). In the sector $\mid \arg (\lambda-x)-$ $\pi / 2 \mid<\pi / 4$ equation (7.12) has the solution $U_{\text {out }}^{x}(x, \lambda)=\left(I+U_{\text {out }}^{x *}(x, \lambda)\right) \mathrm{e}^{(\lambda / 2) J} \lambda^{\left(\left(\sigma-\theta_{\infty}\right) / 4\right) J}$ with $U_{\text {out }}^{x *}(x, \lambda) \ll(\log |x|)^{-1}$ for $\log |x|^{1 / 4}<|\lambda-x|<2|x|^{1 / 2}$, and $U_{\text {in }}^{x}(x, \lambda)=\left(I+U_{\text {in }}^{x *}(x, \lambda)\right) W_{\infty}^{x}(\lambda)$ with $U_{\text {in }}^{x *}(x, \lambda) \ll x^{-1 / 4}$ for $1<|\lambda-x|<\log |x|^{1 / 3}$.

Consider the line joining $\lambda_{\text {st }}$ with a point near $x$ contained in this sector (cf. Fig. 7.2(b)). Then $M_{x}$ is obtained by the matching scheme

$$
Y(x, \lambda) \longleftrightarrow Z_{\mathrm{WKB}}^{x}(x, \lambda) \longleftrightarrow U_{\text {out }}^{x}(x, \lambda) \longleftrightarrow U_{\text {in }}^{x}(x, \lambda)
$$

(cf. Lemma 7.1). Since $\arg x, \arg \lambda \sim \pi / 2$, we may write $\lambda=x(1+(\lambda-x) / x)$ in the domain $|x|^{1 / 2}<|\lambda-x|<2|x|^{1 / 2}$. Using this fact we derive $M_{x}$ as in Theorem 2.10 or 2.11 .

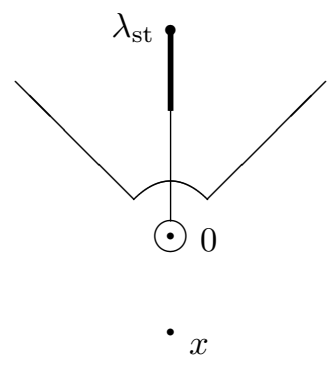

(a) $l_{0}$

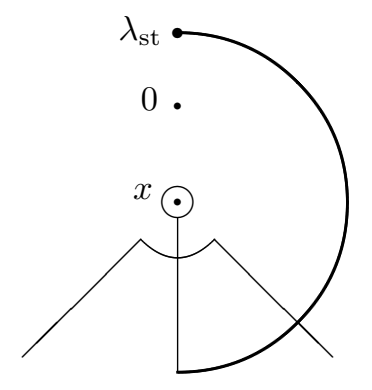

(b) $l_{x}$

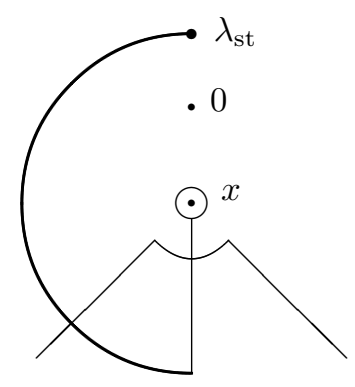

(c) $l_{0} l_{x} l_{0}^{-1}$

Figure 7.3. $l_{0}, l_{x}$ and $l_{0} l_{x} l_{0}^{-1}$.

\subsection{On Remark 2.15}

In the case $\arg x \sim-\pi / 2$, the monodromy matrices are obtained in the same way as above. In the matching procedure to compute $M_{0}^{(-1)}$, we note the fact that $\lambda-x=\mathrm{e}^{\pi \mathrm{i}} x(1-\lambda / x)$ in the domain $|\arg \lambda-\pi / 2|<\pi / 4,|x|^{1 / 2}<|\lambda|<2|x|^{1 / 2}$, since $\arg (\lambda-x) \sim \pi / 2$ (cf. Fig. 7.3(a)). The matrix $M_{x}^{(-1)}$ is obtained by using a curve on the right-hand side of $\lambda=0$ entering into the domain $|\arg (\lambda-x)+\pi / 2|<\pi / 4,|x|^{1 / 2}<|\lambda-x|<2|x|^{1 / 2}$, in which $\lambda=x(1+(\lambda-x) / x)$, 
since $|\arg \lambda+\pi / 2|<\pi / 4$ (cf. Fig. 7.3(b)). A curve on the left-hand side of $\lambda=0$ entering into the domain $|\arg (\lambda-x)-3 \pi / 2|<\pi / 4,|x|^{1 / 2}<|\lambda-x|<2|x|^{1 / 2}$ (cf. Fig. 7.3(c)) corresponds to the expression of $\left(M_{0}^{(-1)}\right)^{-1} M_{x}^{(-1)} M_{0}^{(-1)}$, which is derived by using $\lambda=\mathrm{e}^{2 \pi \mathrm{i}} x(1+(\lambda-x) / x)$ in this domain, since $|\arg \lambda-3 \pi / 2|<\pi / 4$.

\section{Acknowledgements}

The author is grateful to the referees for valuable comments and for bringing the literature [19] to attention; and also appreciation goes to the editor for works of Andrei Kapaev.

\section{References}

[1] Abramowitz M., Stegun I.A. (Editors), Handbook of mathematical functions with formulas, graphs, and mathematical tables, Dover Publications, Inc., New York, 1972.

[2] Andreev F.V., Kitaev A.V., Connection formulae for asymptotics of the fifth Painlevé transcendent on the real axis, Nonlinearity 13 (2000), 1801-1840.

[3] Bonelli G., Lisovyy O., Maruyoshi K., Sciarappa A., Tanzini A., On Painlevé/gauge theory correspondence, Lett. Math. Phys. 107 (2017), 2359-2413, arXiv:1612.06235.

[4] Dubrovin B., Mazzocco M., Monodromy of certain Painlevé-VI transcendents and reflection groups, Invent. Math. 141 (2000), 55-147, math.AG/9806056.

[5] Erdélyi A., Magnus W., Oberhettinger F., Tricomi F.G., Higher transcendental functions, Vol. 1, Bateman Manuscript Project, McGraw-Hill Book Co., New York.

[6] Fedoryuk M.V., Asymptotic analysis. Linear ordinary differential equations, Springer-Verlag, Berlin, 1993.

[7] Fokas A.S., Its A.R., Kapaev A.A., Novokshenov V.Yu., Painlevé transcendents. The Riemann-Hilbert approach, Mathematical Surveys and Monographs, Vol. 128, Amer. Math. Soc., Providence, RI, 2006.

[8] Gamayun O., Iorgov N., Lisovyy O., Conformal field theory of Painlevé VI, J. High Energy Phys. 2012 (2012), no. 10, 038, 25 pages, arXiv:1207.0787.

[9] Gamayun O., Iorgov N., Lisovyy O., How instanton combinatorics solves Painlevé VI, V and IIIs, J. Phys. A: Math. Theor. 46 (2013), 335203, 29 pages, arXiv:1302.1832.

[10] Gromak V.I., On the theory of Painlevé's equations, Differential Equations 11 (1975), 285-287.

[11] Guzzetti D., On the critical behavior, the connection problem and the elliptic representation of a Painlevé VI equation, Math. Phys. Anal. Geom. 4 (2001), 293-377, arXiv:1010.1330.

[12] Guzzetti D., Tabulation of Painlevé 6 transcendents, Nonlinearity 25 (2012), 3235-3276, arXiv:1108.3401.

[13] Its A.R., Novokshenov V.Yu., The isomonodromic deformation method in the theory of Painlevé equations, Lecture Notes in Math., Vol. 1191, Springer-Verlag, Berlin, 1986.

[14] Jimbo M., Monodromy problem and the boundary condition for some Painlevé equations, Publ. Res. Inst. Math. Sci. 18 (1982), 1137-1161.

[15] Jimbo M., Miwa T., Monodromy preserving deformation of linear ordinary differential equations with rational coefficients. II, Phys. D 2 (1981), 407-448.

[16] Kapaev A.A., Asymptotic behavior of the solutions of the Painlevé equation of the first kind, Differential Equations 24 (1988), 1107-1115.

[17] Kapaev A. A., Global asymptotics of the second Painlevé transcendent, Phys. Lett. A 167 (1992), 356-362.

[18] Kapaev A.A., Global asymptotics of the fourth Painlevé transcendent, Steklov Math. Inst. and IUPUI, Preprint \# 96-06, 1996, available at http://www.pdmi.ras.ru/preprint/1996/index.html.

[19] Lisovyy O., Nagoya H., Roussillon J., Irregular conformal blocks and connection formulae for Painlevé V functions, J. Math. Phys. 59 (2018), 091409, 27 pages, arXiv:1806.08344.

[20] Nagoya H., Irregular conformal blocks, with an application to the fifth and fourth Painlevé equations, J. Math. Phys. 56 (2015), 123505, 24 pages, arXiv:1505.02398.

[21] Olver F.W.J., Asymptotics and special functions, Computer Science and Applied Mathematics, Academic Press, New York - London, 1974. 
[22] Shimomura S., Series expansions of Painlevé transcendents near the point at infinity, Funkcial. Ekvac. 58 (2015), 277-319.

[23] Shimomura S., The sixth Painlevé transcendents and the associated Schlesinger equation, Publ. Res. Inst. Math. Sci. 51 (2015), 417-463.

[24] Shimomura S., Critical behaviours of the fifth Painlevé transcendents and the monodromy data, Kyushu J. Math. 71 (2017), 139-185, arXiv:1602.08808.

[25] Sibuya Y., Global theory of a second order linear ordinary differential equation with a polynomial coefficient, North-Holland Mathematics Studies, Vol. 18, North-Holland Publishing Co., Amsterdam - Oxford, 1975.

[26] Takano K., A 2-parameter family of solutions of Painlevé equation (V) near the point at infinity, Funkcial. Ekvac. 26 (1983), 79-113.

[27] Wasow W., Asymptotic expansions for ordinary differential equations, Pure and Applied Mathematics, Vol. 14, Interscience Publishers John Wiley \& Sons, Inc., New York - London - Sydney, 1965.

[28] Wasow W., Linear turning point theory, Applied Mathematical Sciences, Vol. 54, Springer-Verlag, New York, 1985. 